\title{
Computação Incremental e Eficiente de Sequências de Árvores de Componentes
}

\author{
Alexandre Morimitsu \\ DISSERTAÇÃO APRESENTADA \\ $\mathrm{AO}$ \\ Instituto De Matemática e EstatísticA \\ DA \\ Universidade DE SÃo PAUlo \\ PARA \\ OBTENÇÃO DO TÍTULO \\ $\mathrm{DE}$ \\ Mestre em CiênCIAS \\ Programa: Ciência da Computação \\ Orientador: Prof. Dr. Ronaldo Fumio Hashimoto
}

Durante o desenvolvimento deste trabalho o autor recebeu auxílio financeiro do CNPq

São Paulo, outubro de 2015 


\section{Computação Incremental e Eficiente de Sequências de Árvores de Componentes}

Esta versão da dissertação contém as correções e alterações sugeridas pela Comissão Julgadora durante a defesa da versão original do trabalho, realizada em 24/08/2015. Uma cópia da versão original está disponível no

Instituto de Matemática e Estatística da Universidade de São Paulo.

Comissão Julgadora:

- Prof. Dr. Ronaldo Fumio Hashimoto (orientador) - IME-USP

- Prof. Dr. Roberto de Alencar Lotufo - UNICAMP

- Prof. Dr. Paulo André Vechiatto de Miranda - IME-USP 


\section{Agradecimentos}

Primeiramente, aos meus pais e ao meu irmão, por todo o apoio, auxílio e incentivo dados durante este período de estudos na Universidade.

Ao professor Ronaldo Fumio Hashimoto, pela sua paciência e dedicação como orientador durante o final da minha graduação e durante todo o período de Mestrado.

Ao meu colega Wonder Alexandre, por todas as suas contribuições que ajudaram a aprimorar este trabalho.

Aos professores Paulo André Vechiatto de Miranda e Roberto de Alencar Lotufo, pelos seus comentários e sugestões dados durante o exame de qualificação.

Ao $\mathrm{CNPq}$, pelo apoio financeiro, e a todas as pessoas do Instituto e da Universidade que, de forma indireta, permitiram o desenvolvimento desta dissertação.

Por fim, no âmbito pessoal, gostaria de agradecer também o apoio demonstrado pelos meus amigos e parentes, aos quais gostaria de destacar os meus avôs e tios.

A todos vocês, meu muito obrigado! 


\section{Resumo}

\section{MORIMITSU, A. Computação Incremental e Eficiente de Sequências de Árvores de Com-}

ponentes. Dissertação (Mestrado) - Instituto de Matemática e Estatística, Universidade de São Paulo, São Paulo, 2015.

Árvore de componentes é uma forma hierárquica de representar imagens em níveis de cinza baseada nas relações de inclusão dos componentes conexos da imagem. A escolha da vizinhança utilizada para gerar os componentes impacta diretamente na árvore resultante, de forma que uma alteração na escolha da vizinhança pode acarretar em uma alteração na árvore de componentes obtida. Em particular, quando uma sequência de vizinhanças crescentes é usada, os nós das árvores obtidas a partir dessas vizinhanças satisfazem uma relação de inclusão, de forma que se é possível estabelecer relações entre nós de diferentes árvores.

Assim sendo, o principal objetivo desta dissertação consiste no desenvolvimento de um algoritmo eficiente para a construção de uma sequência de árvores de componentes. Para tanto, será introduzida uma classe particular de sequências de vizinhanças, que não apenas satisfaz a propriedade crescente como também permite que as árvores de componentes associadas a ela sejam construídas de forma incremental. Com base nestas propriedades, um novo algoritmo de construção de árvores de componentes associado a esta classe de vizinhanças será proposto. Para analisar a eficiência do algoritmo proposto apresentamos, ao final do texto, alguns resultados práticos e teóricos obtidos com relação ao consumo de tempo e à complexidade computacional.

Palavras-chave: componente conexo, operador conexo, árvore de componentes, conectividade de segunda geração. 


\section{Abstract}

\section{MORIMITSU, A. Incremental and Efficient Computation of Sequences of Component}

Trees. Dissertation - Instituto de Matemática e Estatística, Universidade de São Paulo, São Paulo, 2015 .

Component tree is a hierarchical way of representing gray-level images based on the inclusion relation of the connected components of the image. The choice of the neighborhood used to generate these components directly impacts in the resulting tree: changing the neighborhood used may alter the resulting component tree. In particular, when considering a sequence of increasing neighborhoods, the nodes of the obtained from these neighborhoods will also satisfy an inclusion relation and that will make it possible to link nodes from different trees.

Thus, the main goal of this dissertation is the development of an efficient algorithm to build a sequence of component trees. In order to do that, we will introduce a class of sequences of neighborhood that not only satisfy the increasing property but also makes it possible to incrementally build the trees associated to it. This additional property will guide us to a novel algorithm, which will build the component trees associated to this class of neighborhoods. To show the efficiency of the proposed algorithm, we present some experimental and theoretical results regarding time consumption and computational complexity.

Keywords: connected component, connected operator, component tree, second-generation connectivity 


\section{Sumário}

$\begin{array}{ll}\text { Lista de Abreviaturas } & \text { ix }\end{array}$

Lista de Símbolos $\quad$ xi

Lista de Figuras $\quad$ xiii

Lista de Tabelas $\quad$ xv

1 Introdução $\quad 1$

1.1 Motivação . . . . . . . . . . . . . . . . . . . . . . . . 2

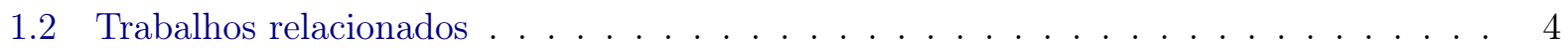

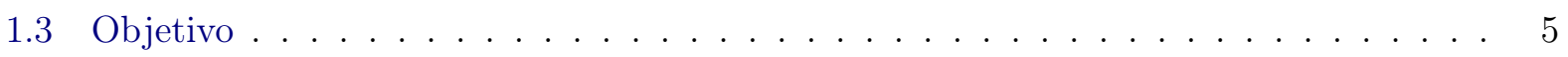

1.4 Organização do Trabalho . . . . . . . . . . . . . . . . . . . . 5

$\begin{array}{lll}2 & \text { Fundamentos } & \mathbf{7}\end{array}$

2.1 Dilatação . . . . . . . . . . . . . . . . . . . . . 7

2.1 .1 Propriedades da dilatação . . . . . . . . . . . . . . . . . . . . 7

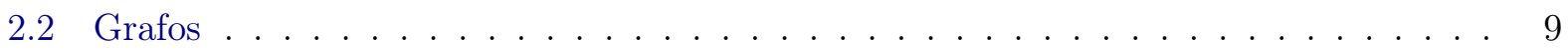

2.2 .1 Definições de árvores . . . . . . . . . . . . . . . . . . . . . . . . . 9

2.3 Imagens . . . . . . . . . . . . . . . . . . . . . . . 10

2.3.1 Representação de imagens através de grafos . . . . . . . . . . . . . . . 10

2.4 Árvore de componentes . . . . . . . . . . . . . . . . . . . . 11

2.4.1 Propriedades dos conjuntos de níveis . . . . . . . . . . . . . . . . 11

2.4.2 Representação compacta da árvore de componentes . . . . . . . . . . . . . . . 12

2.4 .3 Max-tree e min-tree . . . . . . . . . . . . . . . . . . . 13

2.4.4 Informações contidas nos nós de uma max-tree . . . . . . . . . . . . . . . 14

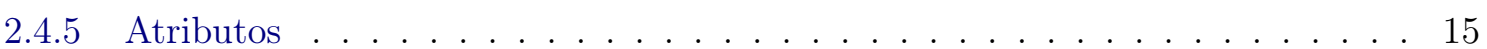

3 Computação Incremental de max-trees $\quad 17$

3.1 Sequências de vizinhanças . . . . . . . . . . . . . . . . . . . 17

3.1.1 Propriedades da sequência gerada $\mathbb{A} \ldots \ldots \ldots \ldots$. . . . . . . . . 17

3.2 Componentes conexos de um conjunto de nível . . . . . . . . . . . . . . . . . 19

3.2.1 Rotulação: obtendo os componentes conexos das vizinhanças da sequência gerada $\mathbb{A} \ldots \ldots \ldots \ldots$

3.2.2 Grafo de rotulações . . . . . . . . . . . . . . . . . . . . . . . . . 22

3.2 .3 Rotulação em níveis de cinza . . . . . . . . . . . . . . . . . . . . 22 
3.2 .4 Alteração de vizinhança . . . . . . . . . . . . . . . . . . . . . . . . . 24

4 Implementação $\quad 27$

4.1 Algoritmo de construção de max-trees . . . . . . . . . . . . . . . . . . . 27

4.1.1 Estrutura de conjuntos disjuntos . . . . . . . . . . . . . . . . . . 27

4.2 Computação eficiente dos rótulos (caso binário) . . . . . . . . . . . . . . . . . . 29

4.2.1 Armazenamento eficiente da função de rotulação . . . . . . . . . . . . . . . . 30

4.2 .2 Deteç̧ão de rótulos inalterados . . . . . . . . . . . . . . . . . . . 30

4.2 .3 Algoritmo . . . . . . . . . . . . . . . . . . . . . . . . . . 31

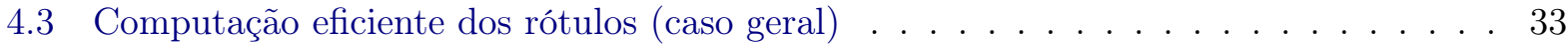

4.3.1 União de CCs na representação compacta . . . . . . . . . . . . . . . . . . . 33

4.4 Algoritmo de rotulação . . . . . . . . . . . . . . . . . . . . . . . 36

4.4 .1 Visão geral . . . . . . . . . . . . . . . . . . . . . . . . 36

4.4.2 Armazenamento eficiente da função de rotulação . . . . . . . . . . . . . . 36

4.4 .3 Detecção de rótulos inalterados . . . . . . . . . . . . . . . . . . . . 37

4.4.4 Processamento ordenado dos níveis de cinza . . . . . . . . . . . . . . 37

4.4 .5 Algoritmo . . . . . . . . . . . . . . . . . . . . . . 38

4.4 .6 Reconstrução de max-trees intermediárias . . . . . . . . . . . . . . . . . . 45

4.4 .7 Canonização . . . . . . . . . . . . . . . . . . . . . 45

4.5 Cálculo de atributos na max-tree . . . . . . . . . . . . . . . . 46

4.5.1 Inversão das ligações da $\max$-tree . . . . . . . . . . . . . . . . . . . . 46

4.5.2 Análise de atributos de nós de outras árvores . . . . . . . . . . . . . . . . 47

4.5.3 Atualização da lista CCsOriginais dos nós . . . . . . . . . . . . . . . . . . 49

5 Resultados Experimentais $\quad 51$

5.1 Comparação da variação de atributos . . . . . . . . . . . . . . . . . . 51

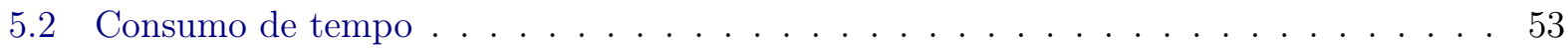

5.2 .1 Atualização das relações de parentesco . . . . . . . . . . . . . . . . . . 53

5.2 .2 Algoritmos posteriores . . . . . . . . . . . . . . . . . . 55

5.3 Complexidade esperada do algoritmo de construção das max-trees . . . . . . . . . . 57

6 Considerações Finais $\quad 59$

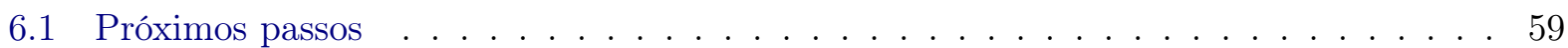

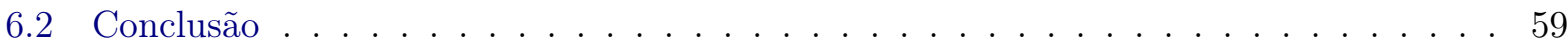

$\begin{array}{ll}\text { Apêndice } & 61\end{array}$

$\begin{array}{lll}\text { A Lista de Publicações } & 63\end{array}$

Referências Bibliográficas $\quad 65$

$\begin{array}{ll}\text { Índice Remissivo } & 67\end{array}$ 


\title{
Lista de Abreviaturas
}

\author{
CC Componente conexo \\ EE Elemento estruturante \\ pixel elemento da imagem (Picture element) \\ ISMM International Symposium on Mathematical Morphology \\ ICDAR International Conference on Document Analysis and Recognition
}




\section{Lista de Símbolos}

$\begin{array}{ll}\mathcal{A} & \text { subconjunto do } \mathbb{Z}^{2}, \text { EE } \\ \mathbb{A} & \text { sequência gerada de elementos estruturantes } \\ \mathcal{B} & \text { subconjunto do } \mathbb{Z}^{2}, \text { EE } \\ \mathbb{B} & \text { sequência geradora e elementos estruturantes } \\ c & \text { Tamanho do ciclo da sequência geradora } \mathbb{B} \\ C & \text { Conjunto } \\ \mathcal{C} & \text { componente conexo } \\ C G & \text { número de vértices em um caminho no grafo } \\ \mathcal{D} & \text { domínio da imagem } \\ E(G) & \text { conjunto de arestas do grafo } G \\ f & \text { imagem } \\ G & \text { grafo } \\ k & \text { índice de um vértice em um caminho de um grafo } \\ \kappa & \text { valor de atributo } \\ K & \text { número de níveis de cinza } \\ \mathcal{K} & \text { número de níveis de cinza } \\ \lambda & \text { limiar (conjunto de nível superior) } \\ \mu & \text { limiar (conjunto de nível inferior) } \\ N & \text { número de elementos em uma sequência de EEs } \\ N R & \text { número de elementos em um caminho } \\ \mathcal{N} & \text { nó de uma maxtree } \\ p & \text { pixel } \\ \pi \mathcal{T}(v, r) & \text { caminho de } v \text { a } r \text { no grafo } \mathcal{T} \\ \mathcal{P} & \text { subconjunto do } \mathbb{Z}^{2}, \text { usado na dilatação } \\ \mathcal{Q} & \text { estrutura de fila de prioridades } \\ r & \text { nó raiz } \\ \mathcal{R} & \text { peso de um vértice ou aresta em um grafo } \\ & \text { conjunto dos números reais } \\ & \\ \mathcal{R} & \end{array}$


$S_{G} \quad$ subgrafo de $G$

$\mathcal{T}$ árvore

$v \quad$ vértice de um grafo

$V(G)$ conjunto de vértices do grafo $G$

$w \quad$ vértice de um grafo

$x \quad$ coordenada $x$ do pixel

$X \quad$ imagem binária, representando um conjunto de nível

$y \quad$ coordenada $y$ do pixel

$\mathbb{Z} \quad$ conjunto dos inteiros 


\section{Lista de Figuras}

1.1 Duas imagens e suas respectivas representações em árvore de componentes sob a vizinhança dada. . . . . . . . . . . . . . . . . . . . . . . . . . . . . . . . 2

1.2 Árvore de componentes das imagens anteriores usando diferentes vizinhanças. Dependendo da escolha, um nó pode representar tanto uma letra quanto uma palavra inteira. A reconstrução de qualquer uma dessas árvores nos leva à imagem original, sem alterações. . . . . . . . . . . . . . . . . . . . . . . . . . . . . . . 2

1.3 Relações de inclusão de CCs entre as árvores com diferentes vizinhanças. . . . . . . . 3

2.1 Exemplo de uma árvore. . . . . . . . . . . . . . . . . . . . . . . . 10

2.2 Exemplo de árvore de componentes para a imagem $f$ e a vizinhança $\mathcal{A}$ apresentadas. Sob a imagem $f$ são apresentados os CCs dos conjuntos de níveis superiores de $f$. Cada CC se torna um nó da árvore de componentes, e as arestas representam as relações de inclusão que os nós satisfazem. . . . . . . . . . . . . . . . . . . . . . . 13

2.3 A árvore de componentes da Fig. 2.2 (esquerda) e sua respectiva max-tree. Em cada nó, os pixels em azul representam os pixels que não estão presentes nos nós filhos, enquanto que os pixels cinzas são herdados dos filhos. . . . . . . . . . . . . . . . . . . 14

3.1 Algumas vizinhanças geradas a partir de um conjunto de EEs. . . . . . . . . . . . . . 18

3.2 Imagem binária para fins de exemplo. . . . . . . . . . . . . . . . . . . . . . . . . . . 20

3.3 Grafo de rotulações $G_{R o t}$ de $X$ do exemplo anterior. . . . . . . . . . . . . . . . . . . 22

3.4 Imagem $f$ em níveis de cinza e uma vizinhança $\mathcal{A}_{1}$. . . . . . . . . . . . . . . . . . . 23

3.5 Grafo de rotulações $G_{R o t}$ da imagem $f$ mostrada na Fig. 3.4. . . . . . . . . . . . . . 23

3.6 Extraindo CCs a partir do grafo de rotulações em níveis de cinza. . . . . . . . . . . . 24

3.7 Imagem $f$ e algumas vizinhanças de $\mathbb{A}$. . . . . . . . . . . . . . . . . . . . . . . . . . . 25

3.8 Rotulação de $f$ para as vizinhanças da imagem 3.7. . . . . . . . . . . . . . . . . . . . 25

3.9 Obtendo CCs a partir do grafo de rotulações. . . . . . . . . . . . . . . . . . . . . . 26

4.1 Representação gráfica da estrutura de conjuntos disjuntos. . . . . . . . . . . . . . . . 27

4.2 Max-tree da imagem $f$. . . . . . . . . . . . . . . . . . . . . . . . . . . . 35

4.3 Simulação do algoritmo de união. . . . . . . . . . . . . . . . . . . . . . . . . . . . . . 35

4.4 Max-tree da imagem $f$ para as vizinhanças $\mathcal{A}_{1}$ e $\mathcal{A}_{2}$. . . . . . . . . . . . . . . . . . . 36

4.5 Árvores de componentes para a imagem $f$ e as vizinhanças $\mathcal{A}_{1}$ e $\mathcal{A}_{2}$ apresentadas. . . 40

4.6 Estruturas de dados representando rt, rtProx e a max-tree. . . . . . . . . . . . . . . 41

4.7 Propagação do rótulo de $a$ para seus vizinhos. . . . . . . . . . . . . . . . . . . . . . . . 42

4.8 Propagação do rótulo de $c$ para seus vizinhos. . . . . . . . . . . . . . . . . . . . . . . 42 
4.9 Propagação do rótulo de $d$ para seus vizinhos. . . . . . . . . . . . . . . . . . . . . . . 42

4.10 Resultado da propagação do rótulo de $d$. . . . . . . . . . . . . . . . . . . . . 43

4.11 Estruturas de dados representando rt, rtProx e a max-tree ao final do passo $i=1 . \quad$. 43

4.12 Propagação do rótulo de $b$ para seus vizinhos no passo $i=2$. . . . . . . . . . . . . . 44

4.13 Max-tree para o passo $i=2$. . . . . . . . . . . . . . . . . . . . . . . . . 44

4.14 Exemplo do efeito do algoritmo de canonização em uma imagem em níveis de cinza. . 46

4.15 Imagem $f$ e a max-tree da imagem para as vizinhanças $\mathcal{A}_{1}$ e $\mathcal{A}_{2}$. . . . . . . . . . . 47

5.1 Imagem binária a fins de exemplo. . . . . . . . . . . . . . . . . . . . . . . . . 52

5.2 Alguns componentes conexos de diferentes árvores para a imagem anterior. . . . . . 52

5.3 Imagem e vizinhança usadas para o primeiro teste de consumo de tempo. . . . . . . 53

5.4 Número de verificações de cada algoritmo para a imagem da Fig. 5.3. . . . . . . . . . 54

5.5 Consumo de tempo de cada algoritmo para a imagem da Fig. 5.3. . . . . . . . . . . . 54

5.6 Imagem e vizinhança usadas para o segundo teste de consumo de tempo. . . . . . . 55

5.7 Número de verificações e consumo de tempo para a Fig. 5.3. . . . . . . . . . . . . . . 55

5.8 Número de verificações e consumo de tempo para a Fig. 5.6. . . . . . . . . . . . . . . 56 


\section{Lista de Tabelas}

5.1 Cálculo da variância da altura para alguns componentes conexos. . . . . . . . . . . 51

5.2 Consumo de tempo para cada algoritmo com relação à Fig. 5.3 . . . . . . . . . . . . 56

5.3 Consumo de tempo para cada algoritmo com relação à Fig. 5.6 . . . . . . . . . . . . . 57 


\section{Capítulo 1}

\section{Introdução}

Árvore de componentes consiste em uma forma hierárquica de representar uma imagem digital na forma de árvore introduzida por Salembier, Oliveras e Garrido [SOG98]. Nesta árvore, nós são representados por componentes conexos (CCs) presentes nos conjuntos de níveis da imagem, enquanto que as relações entre eles são obtidas a partir das relações de inclusão que estes componentes obedecem.

Os componentes obtidos dependem diretamente da vizinhança escolhida e, assim, o uso de diferentes vizinhanças reflete na construção de diferentes árvores. Em particular, levando-se em consideração uma sequência de vizinhanças crescentes, componentes conexos obtidos em uma determinada vizinhança consistem de uniões de componentes conexos obtidos em vizinhanças anteriores, fazendo com que nós de diferentes árvores também sigam uma relação de inclusão. Isso permite o estabelecimento de relações entre nós de árvores distintas e a extração de atributos de regiões que representam componentes conexos originalmente desconexos considerando uma vizinhança menor [PN11].

Com base no que foi apresentado, nesta dissertação será tratado o problema da construção eficiente de uma sequência de árvores obtidas com vizinhanças crescente. Para tanto, nós apresentaremos a teoria desenvolvida, mostrando que existe uma sequência de vizinhanças obtidas por dilatações que satisfaz a propriedade crescente e é propícia para o problema tratado. Com base nesta teoria, desenvolveremos um novo algoritmo que permitirá a construção desta sequência de árvores e mostraremos que as propriedades das vizinhanças escolhidas fazem com que o algoritmo desenvolvido seja, de fato, eficiente. 


\subsection{Motivação}

Como uma possível aplicação do uso de vizinhanças crescentes, considere o exemplo a seguir. Considere as duas imagens binárias da Figura 1.1 e suas respectivas representações na forma de árvore de componentes usando vizinhança $3 \times 3$ (comumente chamado de vizinhança 8):
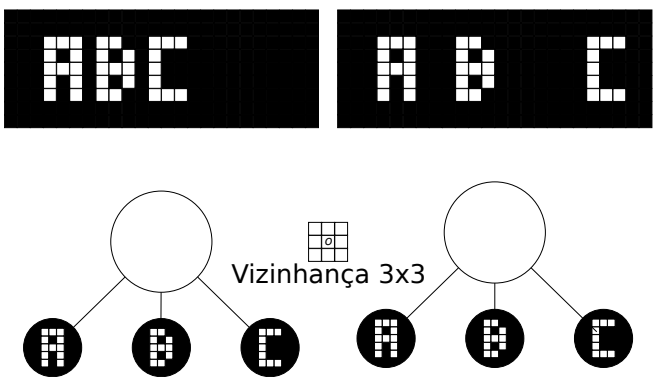

Figura 1.1: Duas imagens e suas respectivas representações em árvore de componentes sob a vizinhança dada.

Apesar dos componentes conexos estarem bem mais próximos na figura da esquerda, as árvores, de um ponto de visto de grafos, são isomorfas. Mesmo considerando os atributos de cada nó, é difícil estabelecer relações entre os componentes conexos, uma vez que muitos atributos são definidos considerando apenas o próprio componente que o define.

Informações com relação à disposição de CCs em uma imagem pode ser uma informação útil dependendo da aplicação em que se está trabalhando. Por exemplo, no problema de localização de textos, distâncias entre letras de uma mesma palavra seguem um padrão relativamente bem comportado. Além disso, é interessante a extração não apenas de atributos com relação a letras isoladas, mas também com relação a toda uma palavra ou frase.

É possível a extração deste tipo de informação fora do domínio da árvore. Por exemplo, dilatandose a imagem para tentar juntar caracteres extraídos da árvore de componentes para a formação de palavras [RM07]. Por outro lado, é possível fazer com que estes mesmos caracteres se juntem apenas alterando a vizinhança para uma vizinhança maior. Esta estratégia tem a vantagem de não alterar a imagem original e usar a mesma estrutura comum (um nó de uma árvore de componentes) para representar tanto os caracteres quanto uma palavra inteira (ver Figura 1.2).
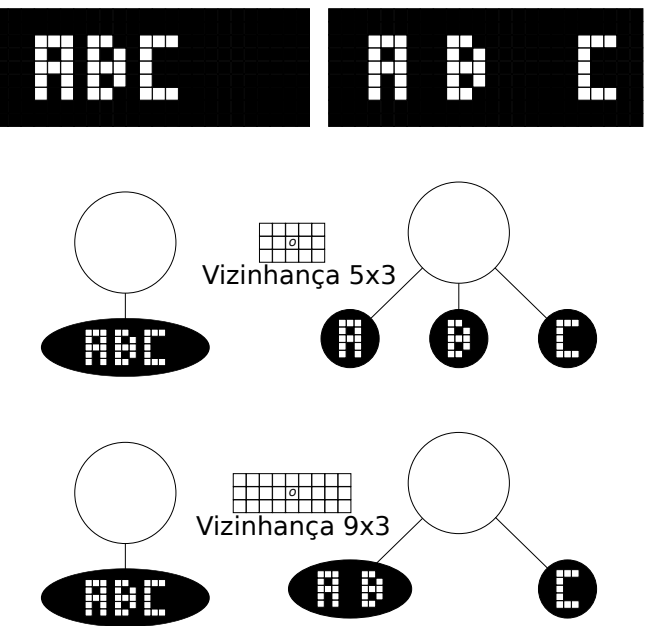

Figura 1.2: Árvore de componentes das imagens anteriores usando diferentes vizinhanças. Dependendo da escolha, um nó pode representar tanto uma letra quanto uma palavra inteira. A reconstrução de qualquer uma dessas árvores nos leva à imagem original, sem alterações.

O exemplo da Fig. 1.2 mostra que é possível utilizar a própria árvore de componentes para a extração de informações de palavras inteiras. Além disso, a alteração da vizinhança em nada altera as propriedades conhecidas sobre árvore de componentes, sendo que é possível construir a árvore e 
extrair atributos dela, da mesma forma que é feito usando vizinhança 4 ou vizinhança 8. Apenas temos que levar em conta que estamos extraindo atributos de componentes que podem não estar mais "grudados". Com a escolha da vizinhança correta, pode-se extrair atributos de uma palavra inteira apenas com a mudança deste parâmetro.

Obviamente, se escolhermos apenas uma vizinhança, temos de fazer uma escolha entre extração apenas de caracteres ou de palavras. Em casos em que existem palavras de espaçamentos diferentes, é possível que não exista uma vizinhança única que consiga corretamente separar palavras em CCs distintos, além de ser difícil estimar de antemão qual a vizinhança que deve ser usada para cada imagem.

Assim sendo, é muito mais interessante tratarmos de um conjunto de vizinhanças. Isso gera um conjunto de árvores de componentes, em que, em algumas é possível que CCs representem apenas letras (e outros ruídos) enquanto que, em outras, alguns CCs representem palavras e outros representem letras separadas. Por fim, com uma vizinhança ainda maior, é possível que as letras separadas tenham se juntado em palavras mas as palavras anteriores tenham se tornado uma frase inteira inclusa em um único componente (ver Figura 1.3).

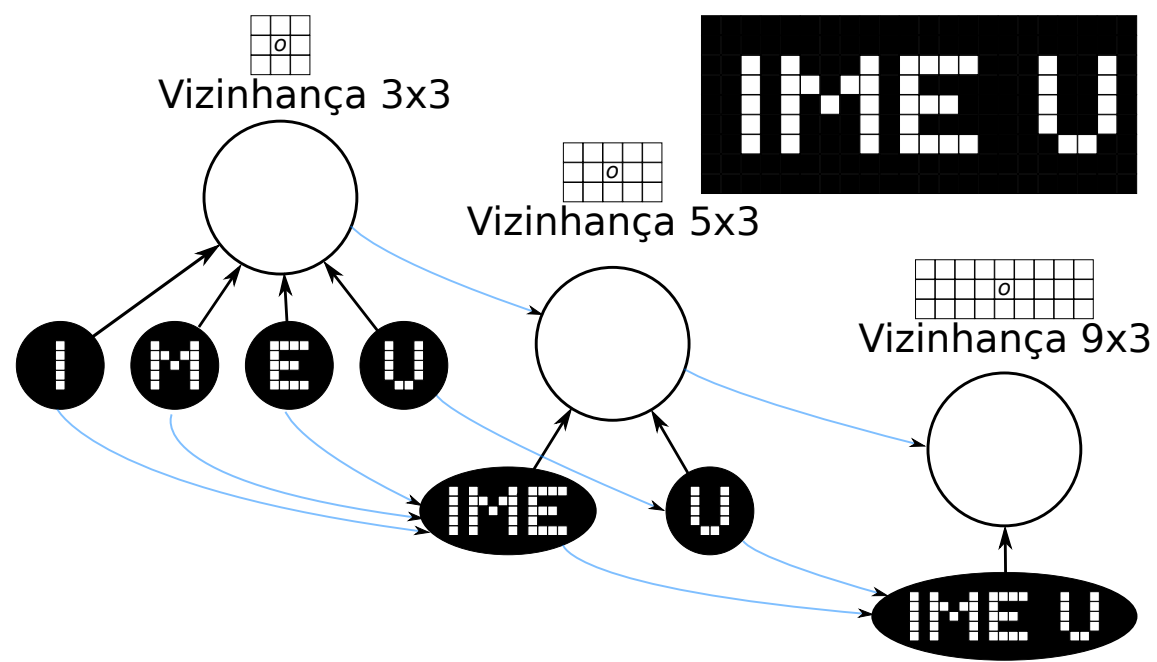

Figura 1.3: Relações de inclusão de CCs entre as árvores com diferentes vizinhanças.

Se escolhermos um "bom" conjunto de vizinhanças, é possível não apenas realizar a extração de características dos nós, mas também o estabelecer relações entre nós de diferentes árvores. Por exemplo, se usarmos uma sequência de vizinhanças que cresce incrementalmente, é possível estimar a distância entre componentes conexos, bastando ver qual a menor vizinhança que faz dois CCs originalmente disjuntos se juntarem em um mesmo CC.

Além disso, se tomarmos duas vizinhanças $\mathcal{A}_{1}$ e $\mathcal{A}_{2}$ satisfazendo $\mathcal{A}_{1} \subseteq \mathcal{A}_{2}$, todo vizinho de um pixel $p$ em $\mathcal{A}_{1}$ também é vizinho de $p$ em $\mathcal{A}_{2}$, o que implica que todo CC da árvore usando $\mathcal{A}_{1}$ é subconjunto de um CC da árvore sob $\mathcal{A}_{2}$. Tal propriedade foi exemplificada na Figura 1.3. Nela, é dada uma imagem binária com as letras "IME U". Os nós consistem dos CCs das árvores com vizinhanças 3x3, 5x3 e 9x3. Arcos pretos representam as relações usuais de inclusão com relação a componentes conexos de uma mesma árvore. Devido às relações de inclusão das vizinhança, existe também inclusões entre CCs das diferentes árvores, indicadas pelos arcos azuis.

A propriedade de inclusão anterior também permite a construção da árvore de componentes sob $\mathcal{A}_{2}$ partindo da árvore sob $\mathcal{A}_{1}$, não sendo necessário computar novamente toda a imagem. Como veremos ao longo do texto, para algumas sequências de vizinhanças em particular, é possível criar um algoritmo eficiente para a montagem das árvores fazendo uso das árvores anteriores. 


\subsection{Trabalhos relacionados}

Como dito anteriormente, árvores de componentes permitem representar uma imagem digital na forma de uma hierarquia de componentes conexos, permitindo não apenas a extração de características de regiões de imagens como também a obtenção das relações de inclusão dentre os componentes. Assim sendo, problemas que envolvem objetos de interesse consistindo de regiões representadas por componentes conexos podem se beneficiar das propriedades desta estrutura. Em particular, aplicações em que é frequente o uso de árvores de componentes incluem localização de textos [LMGN05, RM07, AH10, NM12, SH12, MM13], filtragem conexa de imagens [SOG98, Jon99, SG00, XGN12] ou reconhecimento de objetos [HM11, SRML14], entre outras.

Obviamente, o uso desta estrutura só se tornou popular porque é possível construir a árvore de forma rápida e eficiente para as aplicações mais comuns. Um primeiro algoritmo para a construção da árvore foi apresentado por Salembier et al. [SOG98]. A partir de então, vários outros autores lançaram suas próprias versões de algoritmos para o problema, com o objetivo de aprimorar o consumo de tempo e/ou de memória, aos quais podemos citar os trabalhos de Najman e Couprie [NC06], Berger et al. [BGL $\left.{ }^{+} 07\right]$ e Wilkinson et al. [WGH $\left.{ }^{+} 08\right]$. Uma comparação dos desempenhos destes e outros algoritmos de construção da árvore foi estudada por Carlinet e Géraud [CG13].

Uma ressalva que deve ser feita com relação aos algoritmos citados é que eles têm complexidade que depende do tamanho da vizinhança considerada, e são considerados eficientes porque a maioria das aplicações consideram apenas uma vizinhança pequena (geralmente vizinhança 4 ou vizinhança 8). No nosso caso, como consideramos uma sequência de vizinhanças crescentes, o uso deste algoritmos para construir cada árvore, sem otimizações, acarretaria num tempo de execução alto, por depender tanto do número de árvores quanto do tamanho da vizinhança, que aumenta gradativamente.

$\mathrm{Na}$ realidade, estas vizinhanças crescentes obtidas através de uniões de componentes menores consistem de uma classe particular de uma conectividade conhecida como como conectividade de segunda geração [Ser98, SW09]. Existem diversas formas de se obter vizinhanças que fazem parte desta conectividade. Em particular, a classe de conectividade que usamos neste trabalho é obtida através de dilatações das vizinhanças e é denominado de classe de conectividade baseada em dilatação [BNG03].

Focando-nos a este contexto, o trabalho mais conhecido com relação à construção de árvores condicionado à conectividades de segunda geração é o trabalho de Ouzounis e Winkinson [OW07], em que é passada um imagem na qual os autores chamam de máscara que é responsável por unir ou segmentar componentes conexos da imagem original baseado em como os níveis de cinza da imagem original se comparam com os níveis de cinza da imagem máscara. O trabalho destes autores se difere do nosso no sentido da escolha de como obter os componentes conexos e também se restringem a obter uma única árvore ao invés de uma sequência de árvores.

O trabalho que mais se aproxima ao nosso consiste do trabalho de Passat e Naegel [PN11], que se trata do trabalho em que foi introduzida a ideia de da construção de uma sequência de árvores com vizinhanças crescentes a fim de estabelecer relações entre nós das árvores. Porém, diferentemente desta dissertação, o foco não está na construção eficiente das árvores, mas sim em como usar esta estrutura para resolver problemas de segmentação. Assim como Ouzounis e Winkinson, eles fazem uso de uma conectividade de segunda geração baseada em máscaras ao invés de uma baseada em dilatações.

Assim sendo, se analisarmos este trabalho mais a fundo, a principal contribuição consiste no desenvolvimento de um algoritmo eficiente de construção de uma sequência de árvores de componentes e o estabelecimento de relações entre os nós destas árvores. De acordo com o nosso conhecimento, ainda não existe trabalho na área que faça a exploração destes temas da forma com que tratamos nesta dissertação. As ideias aqui apresentadas serão apresentadas em uma publicação aceita no ISMM (International Symposium on Mathematical Morphology) 2015. As publicações desenvolvidas ao longo do período do desenvolvimento e escrita desta dissertação podem ser vistas no Apêndice ao final do texto. 


\subsection{Objetivo}

Com base em tudo o que foi previamente apresentado, o objetivo final desta dissertação consistirá em apresentar uma teoria que permita o desenvolvimento e a prova de um algoritmo de construção de uma sequência de árvores de componentes. Esta sequência, satisfazendo algumas condições, que serão apresentadas ao longo das seções, permite que a construção seja feita de forma eficiente e incremental, além de permitir a relações de nós de diferentes árvores. Fazendo uso destas propriedades, serão apresentados, aplicações e os resultados obtidos com relação a desempenho, mostrando que a teoria aqui desenvolvida nos leva, de fato, a um algoritmo correto e eficiente.

\subsection{Organização do Trabalho}

Este trabalho é dividido nas seguintes partes: após esta introdução, apresentaremos os conceitos necessários para o entendimento do trabalho, no Capítulo 2. As contribuições deste trabalho começam no Capítulo 3, onde serão mostradas nossas ideias para construirmos a sequência de árvores e também apresentaremos propriedades importantes a fim de provar que a teoria explicitada está, de fato, correta. Com base em toda a teoria vista ao longo destes capítulos, um algoritmo eficiente é então proposto no Capítulo 4. No capítulo seguinte, mostramos alguns resultados experimentais com o uso do algoritmo proposto, exibindo exemplos de possíveis aplicações e análises de tempo e complexidade esperadas. Por fim, no Capítulo 6, serão mostradas a conclusão e a expectativa de trabalhos futuros. 


\section{Capítulo 2}

\section{Fundamentos}

Nesta seção serão vistos os fundamentos do texto, onde apresentaremos os conceitos úteis para a compreensão da teoria que será apresentada nas seções seguintes.

\subsection{Dilatação}

Dilatação [DLfOES03] consiste em uma das operações básicas da Morfologia Matemática e está fortemente relacionada com a Soma de Minkowski.

Dados dois conjuntos $\mathcal{P} \subseteq \mathbb{Z}^{2}$ e $\mathcal{A} \subseteq \mathbb{Z}^{2}$, a dilatação de $\mathcal{P}$ por $\mathcal{A}$, escrita como $\mathcal{P} \oplus \mathcal{A}$, é definida como:

$$
\mathcal{P} \oplus \mathcal{A}=\{p+a: p \in \mathcal{P}, a \in \mathcal{A}\}
$$

O conjunto $\mathcal{A}$ utilizado para dilatar $\mathcal{P}$ é chamado de elemento estruturante(EE).

Da definição apresentada na Equação 2.1, segue que a dilatação satisfaz as seguintes propriedades:

1. Comutatividade: $\mathcal{P} \oplus \mathcal{A}=\mathcal{A} \oplus \mathcal{P}$;

2. Crescente: Se $\mathcal{P} \subseteq \mathcal{P}^{\prime},(\mathcal{P} \oplus \mathcal{A}) \subseteq\left(\mathcal{P}^{\prime} \oplus \mathcal{A}\right)$;

3. Associatividade: $\mathcal{P} \oplus\left(\mathcal{A} \oplus \mathcal{A}^{\prime}\right)=(\mathcal{P} \oplus \mathcal{A}) \oplus \mathcal{A}^{\prime}$.

Dado um conjunto $\mathcal{A}$, sua transposta $\mathcal{A}^{t}$ é definida como:

$$
\mathcal{A}^{t}=\{a:-a \in \mathcal{A}\}=\{-a: a \in \mathcal{A}\}
$$

Segue da Equação 2.2 que a seguinte igualdade é válida:

$$
\left(\mathcal{A}^{t}\right)^{t}=\mathcal{A}
$$

A origem de um EE é definido como a posição $(0,0)$ e denotado pela letra $o$.

\subsubsection{Propriedades da dilatação}

A seguir, serão apresentadas algumas propriedades úteis referentes à dilatação que serão utilizadas no restante do texto. Para simplificação de notação, de agora em diante a dilatação de um elemento $p$ por um elemento estruturante $\mathcal{A}$ será denotado por $p \oplus \mathcal{A}$ ao invés de $\{p\} \oplus \mathcal{A}$.

Proposição 2.1.1. $p \in q \oplus \mathcal{A} \Leftrightarrow q \in p \oplus \mathcal{A}^{t}$.

Demonstração. $p \in q \oplus \mathcal{A} \Leftrightarrow$

$\exists a \in \mathcal{A}$ tal que $p=q+a \Leftrightarrow$

$\exists a \in \mathcal{A}$ tal que $q=p-a \Leftrightarrow$ 
$\exists a \in \mathcal{A}$ tal que $q=p+(-a) \Leftrightarrow$

$\exists a^{\prime}=-a \in \mathcal{A}^{t}$ tal que $q=p+a^{\prime} \Leftrightarrow$

$q \in p \oplus \mathcal{A}^{t}$.

Proposição 2.1.2. $(\mathcal{P} \oplus \mathcal{A})^{t}=\mathcal{P}^{t} \oplus \mathcal{A}^{t}$.

Demonstração. $q \in(\mathcal{P} \oplus \mathcal{A})^{t} \Leftrightarrow$

$-q \in(\mathcal{P} \oplus \mathcal{A}) \Leftrightarrow$

$\exists p \in \mathcal{P}, \exists a \in \mathcal{A}$ tal que $-q=p+a \Leftrightarrow$

$\exists p^{\prime}=-p \in \mathcal{P}^{t}, \exists a^{\prime}=-a \in \mathcal{A}^{t}$ tal que $-q=\left(-p^{\prime}\right)+\left(-a^{\prime}\right) \Leftrightarrow$

$\exists p^{\prime}=-p \in \mathcal{P}^{t}, \exists a^{\prime}=-a \in \mathcal{A}^{t}$ tal que $q=p^{\prime}+a^{\prime} \Leftrightarrow$

$q \in \mathcal{P}^{t} \oplus \mathcal{A}^{t}$

Portanto, como $x \in(\mathcal{P} \oplus \mathcal{A})^{t} \Rightarrow x \in \mathcal{P}^{t} \oplus \mathcal{A}^{t},(\mathcal{P} \oplus \mathcal{A})^{t} \subseteq \mathcal{P}^{t} \oplus \mathcal{A}^{t}$. Por outro lado, $q \in \mathcal{P}^{t} \oplus \mathcal{A}^{t} \Rightarrow$ $q \in(\mathcal{P} \oplus \mathcal{A})^{t}$ implica que $\mathcal{P}^{t} \oplus \mathcal{A}^{t} \subseteq(\mathcal{P} \oplus \mathcal{A})^{t}$. Conclui-se que $(\mathcal{P} \oplus \mathcal{A})^{t}=\mathcal{P}^{t} \oplus \mathcal{A}^{t}$.

Proposição 2.1.3. Dado um conjunto de EEs $\left\{\mathcal{A}_{1}, \mathcal{A}_{2}, \ldots, \mathcal{A}_{N}\right\}$, vale que $\left(\mathcal{A}_{1} \oplus \mathcal{A}_{2} \oplus \ldots \oplus \mathcal{A}_{N}\right)^{t}=$ $\mathcal{A}_{1}^{t} \oplus \mathcal{A}_{2}^{t} \oplus \ldots \oplus \mathcal{A}_{N}^{t}$.

Demonstração. Tal fato pode ser facilmente provado por indução. Para $N=2$, sabemos que a propriedade é válida (caso base, Prop. 2.1.2). Suponha que a condição é satisfeita para os passos anteriores a $i$. Vamos provar que a proposição é válida para o passo $i$ :

$\mathcal{A}_{1}^{t} \oplus \mathcal{A}_{2}^{t} \oplus \ldots \oplus \mathcal{A}_{i}^{t}$

$=\left(\mathcal{A}_{1}^{t} \oplus \mathcal{A}_{2}^{t} \oplus \ldots \oplus \mathcal{A}_{i-1}^{t}\right) \oplus \mathcal{A}_{i}^{t}$

$=\left(\mathcal{A}_{1} \oplus \mathcal{A}_{2} \oplus \ldots \oplus \mathcal{A}_{i-1}\right)^{t} \oplus \mathcal{A}_{i}^{t}$ (Passo de indução)

$=\left(\mathcal{A}_{1} \oplus \mathcal{A}_{2} \oplus \ldots \oplus \mathcal{A}_{i}\right)^{t}$ (Prop. 2.1.2).

Proposição 2.1.4. $\exists p \in \mathcal{P}$ tal que $q \in p \oplus \mathcal{A} \Leftrightarrow q \in(\mathcal{P} \oplus \mathcal{A})$.

Demonstração. $q \in p \oplus \mathcal{A} \Leftrightarrow \exists a \in \mathcal{A}$ tal que $q=p+a$.

Como $p \in \mathcal{P}, a \in \mathcal{A}$ e $q=p+a, q \in \mathcal{P} \oplus \mathcal{A}$. Portanto, vale que, se $\exists p \in \mathcal{P}$ tal que $q \in p \oplus \mathcal{A}, q \in$ $\mathcal{P} \oplus \mathcal{A}$.

A volta é uma consequência direta da definição de dilatação.

Proposição 2.1.5. $\mathcal{P} \cap(q \oplus \mathcal{A})^{t} \neq \emptyset \Leftrightarrow q \in \mathcal{P} \oplus \mathcal{A}$.

Demonstração. $\mathcal{P} \cap(q \oplus \mathcal{A})^{t} \neq \emptyset \Leftrightarrow$ $\exists p$ tal que $p \in \mathcal{P}$ e $p \in(q \oplus \mathcal{A})^{t} \Leftrightarrow$

$\exists p$ tal que $p \in \mathcal{P}$ e $q \in p \oplus \mathcal{A}$ (Prop. 2.1.1)

$\Leftrightarrow q \in \mathcal{P} \oplus \mathcal{A}$ (Prop. 2.1.4).

Proposição 2.1.6. Para qualquer $\mathcal{P}, o \in \mathcal{P} \oplus \mathcal{P}^{t}$.

Demonstração. Tome $a \in \mathcal{P}$. Então, por definição, $-a \in \mathcal{P}^{t}$. Logo, $a+(-a) \in \mathcal{P} \oplus \mathcal{P}^{t} \Rightarrow o \in$ $\mathcal{P} \oplus \mathcal{P}^{t}$.

Proposição 2.1.7. Para qualquer $\mathcal{P}, \mathcal{P} \oplus \mathcal{P}^{t}$ é simétrico.

Demonstração. Para mostrar que $\mathcal{P} \oplus \mathcal{P}^{t}$ é simétrico, temos que mostrar que $-q \in \mathcal{P} \oplus \mathcal{P}^{t}$ para qualquer $q \in \mathcal{P} \oplus \mathcal{P}^{t}$.

Seja $q \in \mathcal{P} \oplus \mathcal{P}^{t}$ um elemento qualquer do conjunto. Então, existem $p \in \mathcal{P}$ e $p^{\prime} \in \mathcal{P}^{t}$ tal que $q=p+p^{\prime}$. Note que $-q=-\left(p+p^{\prime}\right)=-p+\left(-p^{\prime}\right)$.

Mas como $p \in \mathcal{P}$, vale que $b=-p \in \mathcal{P}^{t}$. Além disso, $p^{\prime} \in \mathcal{P}^{t} \Rightarrow b^{\prime}=-p^{\prime} \in\left(\mathcal{P}^{t}\right)^{t}=\mathcal{P}$. Logo, como $-q=b+b^{\prime}, q \in \mathcal{P}^{t} \oplus \mathcal{P} \Rightarrow q \in \mathcal{P} \oplus \mathcal{P}^{t}$, pois $\mathcal{P}^{t} \oplus \mathcal{P}=\mathcal{P} \oplus \mathcal{P}^{t}$.

Proposição 2.1.8. Se $\mathcal{P}$ e $\mathcal{A}$ são simétricos, $\mathcal{P} \oplus \mathcal{A}$ é simétrico.

Demonstração. Seja $q \in \mathcal{P} \oplus \mathcal{A}$ um elemento qualquer do conjunto. Então, existem $p \in \mathcal{P}$ e $a \in \mathcal{A}$ tal que $q=p+a$.

Como $\mathcal{P}$ e $\mathcal{A}$ são simétricos, $-p \in \mathcal{P}$ e $-a \in \mathcal{A}$. Então, $-p+(-a) \in \mathcal{P} \oplus \mathcal{A} \Rightarrow-(p+a) \in$ $\mathcal{P} \oplus \mathcal{A} \Rightarrow-q \in \mathcal{P} \oplus \mathcal{A}$. 


\subsection{Grafos}

Grafos [FKW11] são estruturas utilizadas para representar relações entre um conjunto de elementos. Estes elementos são representados na forma de vértices e as relações entre eles são representadas através de arestas. Assim, um grafo $G$ pode ser definido como um par $G=(V(G), E(G))$, onde $V(G)$ é o conjunto de vértices e $E(G) \subset V(G) \times V(G)$ é o conjunto das arestas. Em alguns casos, serão associados aos vértices e/ou às arestas valores numéricos $\rho \in \mathbb{R}$ que indicam o peso de cada vértice e/ou aresta.

Dado um grafo $G$, definimos que dois vértices $v$ e $w$ são vizinhos se o par $\{v, w\}$ está em $E(G)$. Neste caso, também definimos que a aresta $\{v, w\}$ incide em $v$ e $w$ e que $v$ e $w$ são pontas de $\{v, w\}$. Um caminho em um grafo consiste em uma sequência de vértices $\left(v_{1}, v_{2}, \ldots, v_{C G}\right)$ tal que $\left\{v_{k}, v_{k+1}\right\} \in E(G)$ para todo $1 \leq k<C G$. O comprimento do caminho é definido como $C G-1$, ou seja, o número total de arestas dentro do caminho. Um caminho de comprimento pelo menos 3 que começa e termina no mesmo vértice e não passa por vértices repetidos (exceto pelo primeiro e último) é chamado de ciclo.

Chamamos de subgrafo um grafo $S_{G}=\left(V\left(S_{G}\right), E\left(S_{G}\right)\right)$ em que $V\left(S_{G}\right) \subseteq V(G)$ e $E\left(S_{G}\right) \subseteq$ $E(G)$. Se $E\left(S_{G}\right)$ inclui todas as arestas de $E(G)$ cujos dois extremos incidem em vértices de $V\left(S_{G}\right)$, chamaremos o subgrafo de subgrafo induzido pelo conjunto de vértices $V\left(S_{G}\right)$.

Um grafo é dito conexo se, para qualquer par de vértices $v, w \in V(G)$, existe caminho de $v$ a $w$ em $G$. Um componente conexo (a partir de agora abreviado como CC) de um grafo $G$ consiste em um subgrafo $C_{G}$ de $G$ conexo e maximal, isto é, para qualquer par de vértices $v$ e $w$ em $V\left(C_{G}\right)$, existe um caminho de $v$ a $w$ em $C_{G}$ e para qualquer vértice de $v^{\prime} \notin V\left(C_{G}\right)$, não existe um caminho de $v$ em $G$, qualquer que seja $v \in V\left(C_{G}\right)$.

Um grafo que não contém ciclos é denominado floresta. Uma floresta conexa é denominada árvore.

\subsubsection{Definições de árvores}

Para representar árvores, usaremos o símbolo $\mathcal{T}$. Assim, definimos uma árvore como o grafo $\mathcal{T}=(V(\mathcal{T}), E(\mathcal{T}))$. Nesta dissertação, em se tratando de vértices de árvores, usaremos o nome nó.

Para cada árvore, escolheremos um nó como o nó raiz $r$. Neste caso, também chamaremos o grafo de árvore enraizada em $r$.

Dado um nó qualquer $v \in V(\mathcal{T})$, considere o caminho $\pi_{\mathcal{T}}(v, r)=\left(v=v_{1}, v_{2}, \ldots, v_{C G}=r\right)$ que liga $v$ à raiz $r$. O nó $w$ de $\pi_{\mathcal{T}}(v, r)$ tal que $\{v, w\} \in E(\mathcal{T})$ (ou seja, $w=v_{2}$ ) é chamado de nó pai de $v$, enquanto que $v$ será chamado de filho de $w$. Todos os nós $v_{2}, v_{3}, \ldots, v_{C G}$ de $\pi_{\mathcal{T}}(v, r)$ serão chamados de nós ancestrais de $v$, enquanto que $v$ é descendente de todos eles. Por exemplo, na árvore da Fig. 2.1, se considerarmos que $A$ é o nó raiz, então todos os outros nós serão descendentes de $A$ e $A$ será ancestral de todos eles. Em particular, $A$ é nó pai de $B$ e $C$.

Usando as definições anteriores, o nó raiz passará a ser o nó que não contém nenhum nó pai. Todos os nós que não possuem nós filhos serão chamados de nós folha. No exemplo da Fig. 2.1, $D$ e $E$ são nós folha da árvore e $A$ é o nó raiz.

Chamaremos de subárvore enraizada no nó $v$ o subgrafo de $\mathcal{T}$ induzido pelo conjunto de vértices composto por $v$ e todos os seus descendentes. Por exemplo, a subárvore enraizada em $B$ na figura consiste do subgrafo contendo os nós $B, D$ e $E$ e todas as arestas cujos dois extremos incidem dentro deste subconjunto de vértices. 


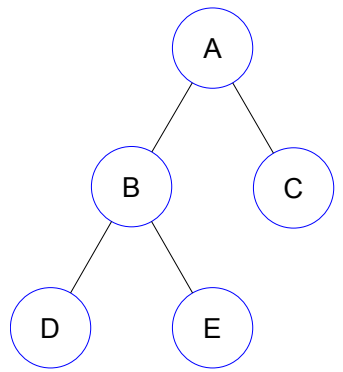

Figura 2.1: Exemplo de uma árvore.

\section{$2.3 \quad$ Imagens}

Uma imagem em níveis de cinza é uma função $f: \mathcal{D} \subset \mathbb{Z}^{2} \rightarrow \mathcal{K}=\{0,1, \ldots, K-1\}$. Cada elemento $p=(x, y) \in \mathcal{D}$ será chamado de pixel.

A aplicação de $f$ em $p$, denotada por $f(p)$, indicará a intensidade do pixel $p$ na imagem $f$.

Neste trabalho, faremos uso de dois tipos de imagem: imagens em níveis de cinza e imagens binárias.

Imagem binárias podem ser representadas de duas formas: a primeira consiste da função análoga à definida em imagens em níveis de cinza para $K=2\left(f: \mathcal{D} \subset \mathbb{Z}^{2} \rightarrow\{0,1\}\right)$. Os pixels $p$ de imagens binárias que satisfizerem $f(p)=1$ serão denominados pixels de objeto, ao passo que pixels que satisfizerem $f(p)=0$ serão denominados pixels de fundo.

Alternativamente, uma imagem binária pode ser representada como um conjunto $X \subseteq \mathcal{D}$, onde os elementos de $X$ são todos os pixels de objeto da imagem.

\subsubsection{Representação de imagens através de grafos}

Para representar um imagem $f$ na forma de um grafo $G_{f}$, consideraremos o conjunto de pixels $f$ como o conjunto de vértices do grafo $\left(V\left(G_{f}\right)=\mathcal{D}\right)$.

O conjunto de arestas $E\left(G_{f}\right)$ será definido a partir de uma vizinhança. As vizinhanças usadas neste trabalho serão definidas usando dilatações: um par de vértices $(v, w)$ pertence à vizinhança $\mathcal{A}$ se $w \in v \oplus \mathcal{A}$. Neste caso, diremos que $v$ é vizinho de $w$ sob a vizinhança $\mathcal{A}$. Assim, dado um EE $\mathcal{A}$, o conjunto de arestas pode ser definido como o conjunto de todos os pares vizinhos sob $\mathcal{A}$, isto é, $E\left(G_{f}\right)=\{(p, q): p \in \mathcal{D}, q \in \mathcal{D}$ e $q \in p \oplus \mathcal{A}\}$. Exemplos normalmente usados de vizinhança incluem vizinhança-4 $(\mathcal{A}=\{(0,0),(-1,0),(1,0),(0,1),(0,-1)\})$ e vizinhança-8 $(\mathcal{A}=\{(0,0),(-1,0),(1,0),(0,1),(0,-1),(-1,-1),(-1,1),(1,1),(1,-1)\})$. Quando estiver claro pelo contexto que estamos usando um EE para definirmos uma vizinhança, usaremos os termos EE e vizinhança como sinônimos.

Tendo definido a vizinhança, podemos representar uma imagem como um grafo $G_{f}$ e podemos aplicar os conceitos de vizinhança e conexidade para imagens. O conceito de CCs, por outro lado, será usado apenas em imagens binárias. Definiremos como componente conexo de uma imagem binária um subconjunto maximal de pixels conexos de objeto. 


\section{4 Árvore de componentes}

Árvore de componentes [SOG98] é uma forma hierárquica de representar os CCs da decomposição de uma imagem em níveis de cinza em imagens binárias. A cada uma dessas imagens binárias geradas nesta decomposição é dado o nome de conjunto de nível.

Dada uma imagem em níveis de cinza $f$, o conjunto de nível superior $\lambda$, denotado ${ }^{1}$ por $X_{\lambda}(f)$, consiste no conjunto de elementos $p \in \mathcal{D}$ de intensidade maior ou igual a $\lambda$, isto é:

$$
X_{\lambda}(f)=\{p \in \mathcal{D}: f(p) \geq \lambda\}
$$

Analogamente, definimos o conjunto de nível inferior $\mu$ de $f$ como:

$$
X^{\mu}(f)=\{p \in \mathcal{D}: f(p) \leq \mu\}
$$

Nesta seção, vamos nos restringir apenas a propriedades relacionadas aos conjuntos de níveis superiores. Como existe uma dualidade entre as duas definições, qualquer propriedade que valha para um conjunto vale para o outro conjunto de forma dual.

É possível reconstruir $f$ a partir do conjunto de níveis superiores através da seguinte equação:

$$
f(p)=\max \left\{\lambda: p \in X_{\lambda}(f)\right\}
$$

\subsubsection{Propriedades dos conjuntos de níveis}

As seguintes proposições são consequências das definições de conjuntos de níveis. As propriedades apresentadas nesta seção são conhecidas e algumas não serão provadas.

Proposição 2.4.1. Dados uma imagem em níveis de cinza $f$ e um pixel $p \in \mathcal{D}$, o menor $C C$ (com relação ao número de elementos) que contém $p$ entre todos os conjuntos de níveis $X_{\lambda}, 0 \leq \lambda \leq 255$ é o $C C$ que contém $p$ no conjunto de nivel $f(p)$ (podem haver outros CC com mesmo número de elementos em outros niveis, mas nunca CCs com número de elementos menor contendo $p$ ).

Proposição 2.4.2. O conjunto de niveis superiores é decrescente, ou seja, dado $0 \leq \alpha<\beta \leq$ $255, X_{\beta} \subseteq X_{\alpha}$.

Proposição 2.4.3. Seja $\mathcal{A}$ o EE usado para definir a vizinhança nos quais os CCs são definidos. Tome um indice $0<\lambda \leq 255$ e um $C C \mathcal{C}_{1} \in X_{\lambda}(f)$. Então, só existirá um $C C \mathcal{C}_{2} \in X_{\lambda-1}(f)$ tal que $\mathcal{C}_{1} \subseteq \mathcal{C}_{2}$.

Demonstração. Suponha por contradição que existam dois $\operatorname{CCs} \mathcal{C}_{2}$ e $\mathcal{C}_{3}$ disjuntos em $X_{\lambda-1}(f)$ tais que ambos contenham $\mathcal{C}_{1}$ sob $\mathcal{A}$. Então, para qualquer elemento $p$ de $\mathcal{C}_{1}, p$ está tanto em $\mathcal{C}_{2}$ quanto em $\mathcal{C}_{3}$. Logo, $p \in \mathcal{C}_{2} \cap \mathcal{C}_{3}$, o que implica que $\mathcal{C}_{2}$ e $\mathcal{C}_{3}$ não são disjuntos. Logo, não podem existir dois CCs em $X_{\lambda-1}$ disjuntos contendo $\mathcal{C}_{1}$, implicando que $\mathcal{C}_{2}$ é único.

A Prop. 2.4.3 nos indica que existe uma relação de inclusão de CCs entre níveis consecutivos. Assim sendo, denote por $\left(\mathcal{C}_{(\lambda, \mathcal{A})}, \lambda\right)$ o par $\left(\mathrm{CC}\right.$, nível), onde $\mathcal{C}_{(\lambda, \mathcal{A})}$ é um $\mathrm{CC}$ do conjunto de nível superior $X_{\lambda}$ sob $\mathcal{A}$. Usando esta notação, definimos os seguintes conjuntos:

$$
\begin{gathered}
C C_{\lambda}(f, \mathcal{A})=\left\{\mathcal{C}_{(\lambda, \mathcal{A})}: \mathcal{C}_{(\lambda, \mathcal{A})} \in X_{\lambda}(f)\right\} \\
C X(f, \mathcal{A})=\left\{\left(\mathcal{C}_{(\lambda, \mathcal{A})}, \lambda\right): \mathcal{C}_{(\lambda, \mathcal{A})} \in C C_{\lambda}(f, \mathcal{A}), 0 \leq \lambda \leq 255\right\}
\end{gathered}
$$

Denotando por $\mathcal{C}_{(\lambda, \mathcal{A})}(p)$ o CC de $X_{\lambda}$ sob $\mathcal{A}$ contendo $p\left(\mathcal{C}_{(\lambda, \mathcal{A})}(p)=\emptyset\right.$ se $\left.p \notin X_{\lambda}\right)$, é possível organizar os CCs de uma imagem em níveis de cinza de forma hierárquica. Definiremos o grafo de níveis superiores $G_{\text {sup }}=\left(V_{\text {sup }}, E_{\text {sup }}\right)$ da seguinte forma:

\footnotetext{
${ }^{1} \mathrm{~A}$ notação subscrita para conjuntos superiores é usada por Monasse e Guichard[MG00]
} 
Definição 2.4.4. $G_{\text {sup }}=\left(V_{\text {sup }}, E_{\text {sup }}\right)$ onde:

1. $V_{\text {sup }}=C X(f, \mathcal{A})$;

2. $E_{\text {sup }}=\left\{\left\{\left(\mathcal{C}_{(\lambda, \mathcal{A})}(p), \lambda\right),\left(\mathcal{C}_{(\lambda-1, \mathcal{A})}(p), \lambda-1\right)\right\}: p \in \mathcal{D}, 0<\lambda \leq 255\right\}$.

Ou seja, $V_{\text {sup }}$ é o conjunto de todos os pares (CC, i) para todos os níveis $0 \leq i \leq 255$ e existe aresta no grafo $G_{\text {sup }}$ sempre que há uma relação de inclusão entre dois CCs de níveis consecutivos. Uma consequência da definição do grafo de níveis superiores é a seguinte:

Proposição 2.4.5. Para qualquer imagem $f$ e qualquer $E E \mathcal{A}$, o grafo de níveis superiores $G_{\text {sup }}$ definido a partir de $f$ e $\mathcal{A}$ não contém ciclos.

Proposição 2.4.6. Dados uma imagem $f$ e um $E E \mathcal{A}$, se o conjunto de nível superior $X_{0}(f)$ consiste em apenas um $C C$ contendo todo o dominio da imagem, o grafo de niveis superiores $G_{\text {sup }}$ definido a partir de $f$ e $\mathcal{A}$ é uma árvore.

Demonstração. Seja $\mathcal{C}_{0}$ o CC de nível 0. Se $\mathcal{C}_{0}$ contém toda a imagem, então todos os outros CCs dos outros conjuntos de níveis serão subconjunto de $\mathcal{C}_{0}$, fazendo com que sempre exista caminho de qualquer $\mathrm{CC}$ a $\mathcal{C}_{0}$. Isso implica que há caminho entre qualquer par de vértices do grafo. Como também sabemos, pela Prop. 2.4.5, que não há ciclos no grafo, $G_{\text {sup }}$ é uma árvore.

Se a Prop. 2.4.6 anterior for não satisfeita, o grafo $G_{\text {sup }}$ será uma floresta e não será possível estabelecer relações entre alguns nós. Desta forma, nos restringiremos apenas aos casos em que a Prop. 2.4.6 vale e o grafo de níveis superiores é uma árvore. Neste caso, é dado ao grafo o nome de árvore de componentes e sua raiz consiste do $\mathrm{CC}$ do conjunto de nível 0 , de forma que a relação de parentesco da árvore agora representa as relações de inclusões dos CCs.

Um exemplos de árvore de componentes é apresentada na Figura 2.2.

\subsubsection{Representação compacta da árvore de componentes}

Se estivéssemos usando apenas os CCs dos conjuntos de níveis de $f$ ao invés do par (CC, nível) para representar os nós da árvore de componentes, possivelmente evitaríamos muitos nós repetidos, já que é provável que um mesmo CC esteja representado em vários conjuntos de níveis distintos.

É interessante evitar a inclusão de nós repetidos na hora de representar a árvore em memória. Porém, temos que tomar o cuidado de lembrar que, nesta representação, alguns CCs originais estão implicitamente representados por um único nó.

Assim, defina o conjunto $C C(f, \mathcal{A})$ como:

$$
C C(f, \mathcal{A})=\left\{\mathcal{C}_{(\lambda, \mathcal{A})}: \mathcal{C}_{(\lambda, \mathcal{A})} \in C C_{\lambda}(f, \mathcal{A}), 0 \leq \lambda \leq 255\right\}
$$

Ou seja, $C C(f, \mathcal{A})$ é o conjunto de todos CCs de todos os conjuntos de níveis de $f$. Usando esta definição, defina o grafo de níveis maximais $G_{\max }$ da seguinte forma:

Definição 2.4.7. $G_{\max }=\left(V_{\max }, E_{\max }\right)$ onde:

1. $V_{\max }=C C(f, \mathcal{A})$;

2. $E_{\max }=\left\{\left\{\mathcal{C}_{1}, \mathcal{C}_{2}\right\}: \mathcal{C}_{1}, \mathcal{C}_{2} \in V_{\max }, \mathcal{C}_{1} \subset \mathcal{C}_{2}\right.$ e $\nexists \mathcal{C}_{3} \in V_{\text {max }}$ tal que $\left.\mathcal{C}_{1} \subset \mathcal{C}_{3} \subset \mathcal{C}_{2}\right\}$.

Este grafo $G_{\max }$ pode ser visto como uma compressão do grafo de níveis superiores $G_{\text {sup }}$, onde o conjunto de vértices de mesmo CC mas níveis $\lambda$ diferentes são todos comprimidos em um único nó em $G_{\max }$. A partir deste fato é fácil verificar que as proposições a seguir também valem:

Proposição 2.4.8. Para qualquer imagem $f$ e qualquer $E E \mathcal{A}$, o grafo de níveis maximais $G_{\max }$ definido a partir de $f$ e $\mathcal{A}$ não contém ciclos. 


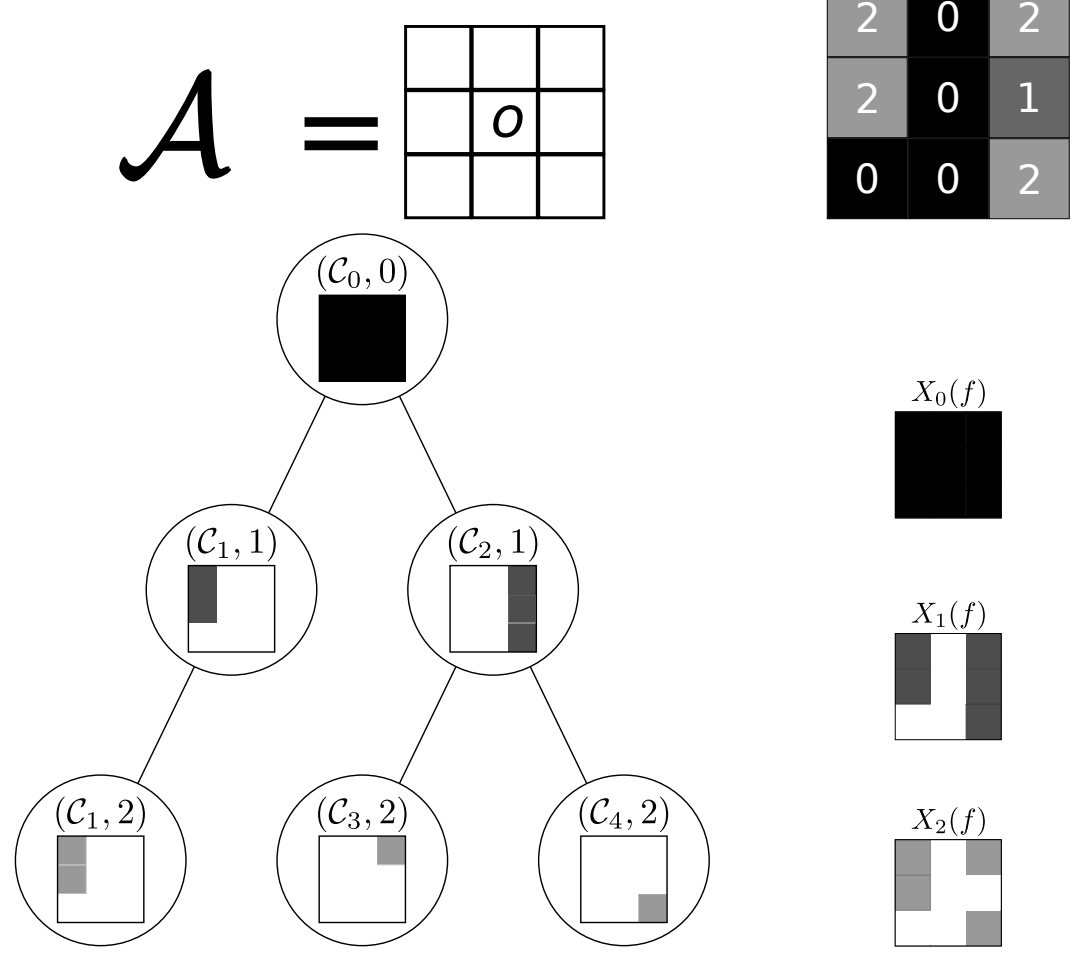

Figura 2.2: Exemplo de árvore de componentes para a imagem $f$ e a vizinhança $\mathcal{A}$ apresentadas. Sob a imagem $f$ são apresentados os CCs dos conjuntos de niveis superiores de $f$. Cada CC se torna um nó da árvore de componentes, e as arestas representam as relações de inclusão que os nós satisfazem.

Proposição 2.4.9. Dados uma imagem $f$ e um $E E \mathcal{A}$, se o conjunto de nível superior $X_{0}(f)$ consiste em apenas um $C C$ contendo todo o dominio da imagem, o grafo de níveis maximais $G_{\max }$ definido a partir de $f$ e $\mathcal{A}$ é uma árvore.

Como vimos na Seção 2.2.1, se o grafo de níveis maximais for uma árvore, a escolha de um nó raiz permite o uso de várias nomenclaturas para referirmos os nós do grafo. Assim como antes, a escolha natural para a raiz é o nó que representa o CC de $X_{0}(f)$, pois este é o CC que contém todos os outros CCs existentes e faz com que as relações de parentesco da árvore respeitem as relações de inclusão de CCs.

\subsubsection{Max-tree e min-tree}

O uso da representação vista na Def. 2.4.7 permite uma redução de espaço necessário em memória para armazenamento da árvore de componentes. Porém, mesmo para nós com pais diferentes, também é possível representar os componentes da árvore usando um número reduzido de informações com relação aos pixels presentes em cada nó: sabemos que todos os pixels de um nó filho estão contidos no nó pai. Assim, armazenar apenas os pixels que estão no nó pai mas não estão nos nós descendentes é suficiente para a reconstrução do CC que este nó representa: é suficiente coletarmos o conjunto de pixels armazenados nos nós descendentes e unirmos com o conjunto de nós armazenados no nó pai.

Em outras palavas, a subárvore enraizada em um nó $\mathcal{N}$ é suficiente para reconstruir o CC que ele representa. Como isso vale para qualquer nó da árvore, podemos usar esta forma econômica de armazenar os pixels para todos os nó. A esta forma compacta e econômica de armazenar os nós de uma árvore de componentes é dada o nome de max-tree (no caso de conjuntos de níveis superiores. Quando usamos conjuntos de níveis inferiores, esta árvore compacta recebe o nome de $\boldsymbol{m i n}$-tree). 
A partir de agora, quando nos referirmos à palavra "árvore", estaremos nos referindo a max-trees. A Fig. 2.3 apresenta a max-tree da árvore de componentes do exemplo anterior.
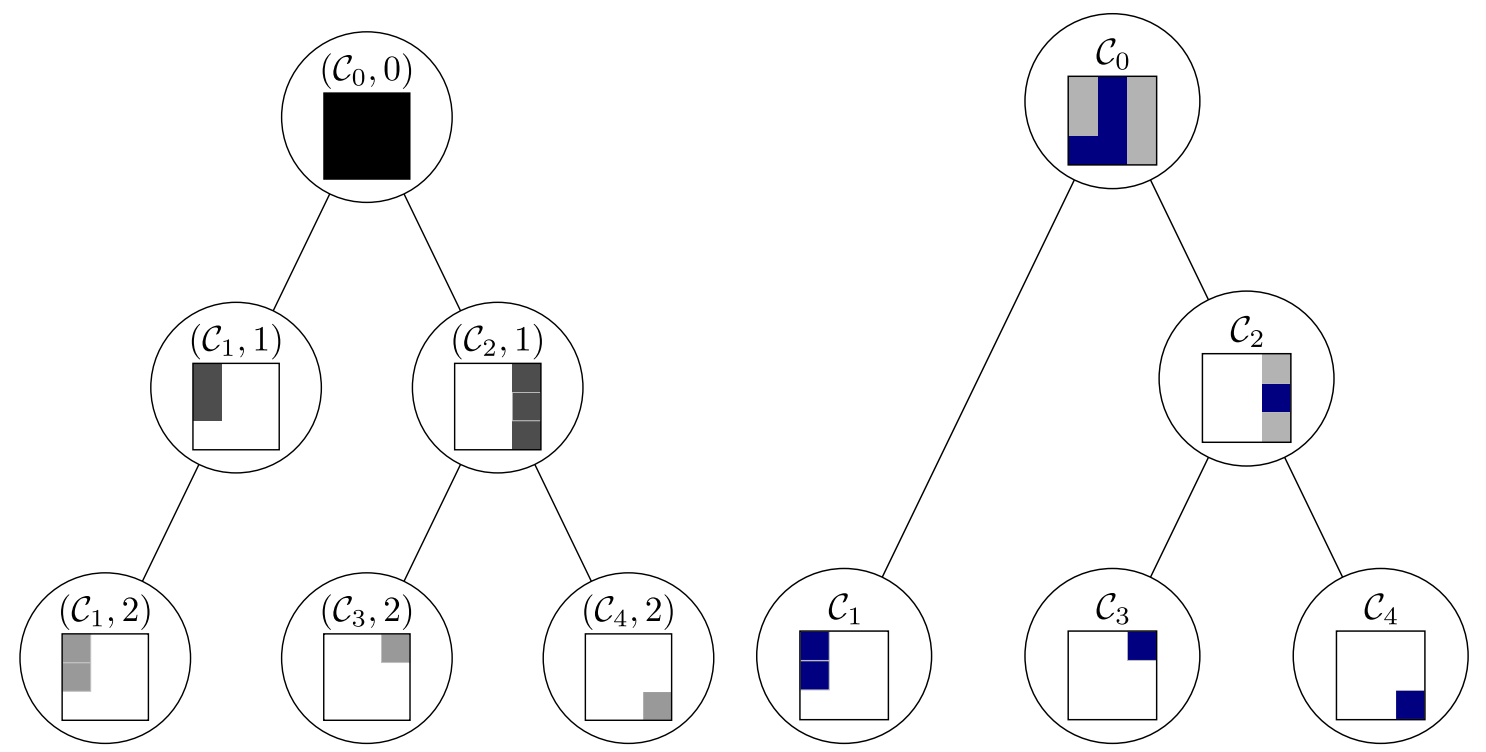

Figura 2.3: A árvore de componentes da Fig. 2.2 (esquerda) e sua respectiva max-tree. Em cada nó, os pixels em azul representam os pixels que não estão presentes nos nós filhos, enquanto que os pixels cinzas são herdados dos filhos.

Para denotar uma max-tree construída sob uma imagem $f$ usando o EE $\mathcal{A}$, usaremos a notação $\mathcal{T}(f, \mathcal{A})$. A letra $\mathcal{N}$ será usada para denotar nós de uma max-tree.

\subsubsection{Informações contidas nos nós de uma max-tree}

A fim de poder reconstruir cada $\mathrm{CC}$ dos conjuntos de níveis da imagem original $f$, de um ponto de vista de estrutura de dados, podemos armazenar o conjunto de pixels dentro do próprio nó. Seja $\mathcal{N}$ um nó da max-tree $\mathcal{T}, C C(\mathcal{N})$ o conjunto de pixels do CC que o nó $\mathcal{N}$ representa e pixels $(\mathcal{N})$ o conjunto de pixels armazenados em $\mathcal{N}$. Então, pixels $(\mathcal{N})$ pode ser formalmente definido como:

$$
\operatorname{pixels}(\mathcal{N})= \begin{cases}C C(\mathcal{N}), & \text { se } \mathcal{N} \text { é nó folha } \\ C C(\mathcal{N}) \backslash\left(\bigcup_{\mathcal{N}_{k} \text { filho de } \mathcal{N}} C C\left(\mathcal{N}_{k}\right)\right) & \text { c.c. }\end{cases}
$$

Como cada pixel é armazenado apenas uma vez, podemos usar os pixels $p \in \mathcal{D}$ para referenciar univocamente os nós de $\mathcal{T}$ :

$$
\mathcal{N}_{\mathcal{T}}(p):=\text { o nó de } \mathcal{T} \text { tal que } p \in \operatorname{pixels}\left(\mathcal{N}_{\mathcal{T}}\right) .
$$

Segue da definição de pixels $(\mathcal{N})$ a seguinte propriedade:

Proposição 2.4.10. Dado um nó $\mathcal{N}$ qualquer de uma max-tree $\mathcal{T}$, para qualquer par de pixels $(p, q)$ em pixels $(\mathcal{N}), f(p)=f(q)$.

Se quisermos reconstruir a imagem original, além da informação dos pixels, é necessário também armazenar um nível de cinza para cada nó. De acordo com a Prop. 2.4.1, é suficiente associar ao nível de cinza do nó $\mathcal{N}, f(\mathcal{N})$, o valor de qualquer $f(p)$ para um $p \in$ pixels $(\mathcal{N})$.

Essa associação de níveis de cinza também permite saber os níveis de cinza onde haviam nós iguais que foram eliminados pela representação compacta:

Proposição 2.4.11. Dados dois nós $\mathcal{N}_{1}$ e $\mathcal{N}_{2}$ de uma max-tree $\mathcal{T}$ com $\mathcal{N}_{1}$ pai de $\mathcal{N}_{2}$, para todo de nivel de cinza $f\left(\mathcal{N}_{2}\right)<k<f\left(\mathcal{N}_{1}\right)$, havia um $C C$ igual a $C C\left(\mathcal{N}_{2}\right)$ eliminado na representação compacta. 
Portanto, é possível recuperar a árvore original usando uma árvore representada na forma compacta e vice-versa.

\subsubsection{Atributos}

Além do nível de cinza é interessante armazenar, para cada nó, outros valores numéricos que permitem extrair características do CC que o nó representa. Chamaremos tais valores numéricos de atributos.

Seja $\mathcal{C}$ um componente conexo. Então, alguns exemplos de atributos extraídos a partir de $\mathcal{C}$ incluem:

- Mínimo $x \kappa_{x \min }(\mathcal{C})$ : a coordenada $x$ do pixel $p=\left(x_{p}, y_{p}\right) \in \mathcal{C}$ com $x_{p}$ mínimo.

Analogamente, pode-se definir os atributos $x \max$, ymin e ymax.

- Altura $\kappa_{\text {altura }}(\mathcal{C})$ : a diferença na coordenada $y$ dentre os pixels com maior e menor coordenadas $y$ dentro de $\mathcal{C}\left(\kappa_{\text {altura }}(\mathcal{C})=\kappa_{\text {xmax }}(\mathcal{C})-\kappa_{\text {xmin }}(\mathcal{C})+1\right)$.

- Posição do centroide $\kappa_{\text {centroide }}(\mathcal{C})$ : as coordenadas $(x, y)$ do centroide de $\mathcal{C}$.

- Área $\kappa_{\text {area }}(\mathcal{C})$ : número de pixels da região $\mathcal{C}$.

Existem inúmeros outros atributos que podem ser extraídos para cada CC, tais como perímetro ou volume. A escolha dos atributos dependem, entre outros fatores, da aplicação em que se está trabalhando e a complexidade necessária para se obtê-los. Para esta dissertação, estes atributos são suficientes para a compreensão das aplicações que serão apresentadas na Seção 5 . 


\section{Capítulo 3}

\section{Computação Incremental de max-trees}

Tal como explicado no Capítulo 1, é interessante definirmos uma sequência de vizinhanças crescentes, de forma que algumas propriedades possam ser aproveitadas na computação das maxtrees. Assim, apresentaremos nesta seção a teoria desenvolvida para se obter as vizinhanças que usaremos, mostrando as boas propriedades que elas satisfazem e como elas podem servir de base para o desenvolvimento de um algoritmo de construção das árvores.

\subsection{Sequências de vizinhanças}

Em princípio, qualquer sequência $\mathbb{A}^{\prime}=\left(\mathcal{A}_{1}^{\prime}, \ldots, \mathcal{A}_{N}^{\prime}\right)$ que satisfaça $\mathcal{A}_{i-1}^{\prime} \subseteq \mathcal{A}_{i}^{\prime}, 2 \leq i \leq N$ serviria para termos a propriedade de que CCs possam ser unidos conforme o índice $i$ aumenta. No entanto, conforme veremos ao longo dessa seção, existe uma classe em particular de vizinhanças que possui algumas propriedades extras, que permitirão uma computação mais eficiente das árvores.

Antes de definir tal sequência, construiremos uma sequência auxiliar de EEs $\mathbb{B}=\left(\mathcal{B}_{1}, \ldots, \mathcal{B}_{N}\right)$, que chamaremos de sequência geradora. Por construção, requiriremos que todos estes $\mathcal{B}_{i}, 1 \leq i \leq$ $N$ contenham a origem.

Definiremos agora uma sequência de EEs $\mathbb{A}=\left(\mathcal{A}_{1}, \ldots, \mathcal{A}_{N}\right)$ que será construída a partir da sequência geradora $\mathbb{B}$. Chamaremos a sequência $\mathbb{A}$ de sequência gerada. A sequência gerada $\mathbb{A}$ será a sequência usada para definir as vizinhanças usadas nas árvores e será definida da seguinte forma:

Definição 3.1.1. Dada uma sequência geradora $\mathbb{B}=\left(\mathcal{B}_{1}, \ldots, \mathcal{B}_{N}\right)$, definimos a sequência gerada $\mathbb{A}=\left(\mathcal{A}_{1}, \ldots, \mathcal{A}_{N}\right)$ como:

1. $\mathcal{A}_{1}=\mathcal{B}_{1} \oplus \mathcal{B}_{1}^{t}$

2. $\mathcal{A}_{i}=\mathcal{A}_{i-1} \oplus \mathcal{B}_{i} \oplus \mathcal{B}_{i}^{t}, 2 \leq i \leq N$.

Como as vizinhanças usadas serão definidas a partir de dilatações, ocorre que algumas vizinhanças não podem ser geradas. (por exemplo, vizinhança 4 não pode ser obtida). Por outro lado, ainda é possível de se gerar um grande conjunto de sequências a partir da definição acima, tais como qualquer vizinhança de formato retangular. A Fig. 3.1 apresenta exemplos de 3 vizinhanças obtidas a partir de alguns elementos estruturantes pré-determinados. Para esta figura, $\mathcal{A}_{i}$ e $\mathcal{B}_{i}$ indicam os EEs considerados para $1 \leq i \leq N$. À esquerda da Fig. 3.1, é mostrada a sequência de EEs quadra$\operatorname{dos}\left(\mathcal{A}_{i}\right.$ tem lado $\left.2 i+1\right)$. No exemplo do meio, é gerada uma sequência de EEs em forma de Disco $\left(\mathcal{A}_{i}\right.$ é a vizinhança na forma de disco com raio $\left.2 i\right)$. A vizinhança mais a direita indica as mesmas vizinhanças usadas na Fig. 1.3.

\subsubsection{Propriedades da sequência gerada $\mathbb{A}$}

Tendo em vista a definição e alguns exemplos de sequências que podem ser geradas, apresentamos em seguida algumas propriedades que a sequência $\mathbb{A}$ definida satisfaz. Primeiramente, mostramos que os elementos da sequência gerada $\mathbb{A}$ satisfazem a propriedade de inclusão: 

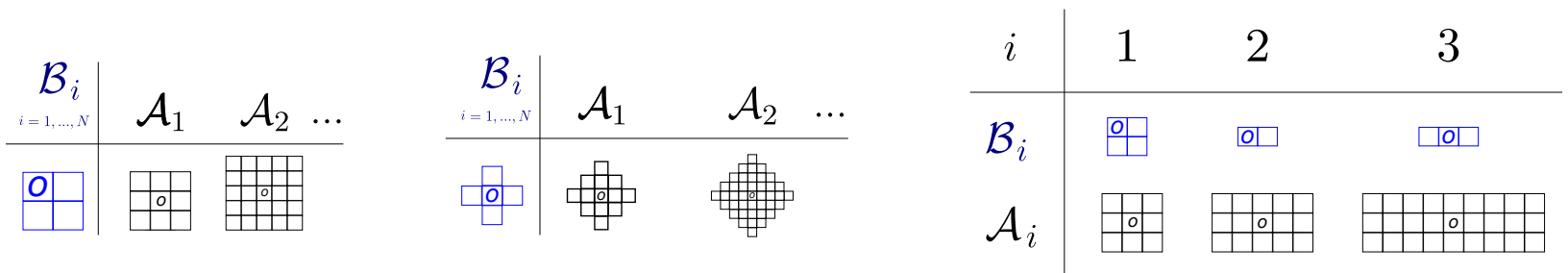

Figura 3.1: Algumas vizinhanças geradas a partir de um conjunto de EEs.

Proposição 3.1.2. Para qualquer sequência gerada $\mathbb{A}=\left(\mathcal{A}_{1}, \ldots, \mathcal{A}_{N}\right)$, vale que $\mathcal{A}_{i-1} \subseteq \mathcal{A}_{i}, 2 \leq$ $i \leq N$.

Demonstração. Para provar esta propriedade, vamos usar outras propriedades vistas na seção 2.1: primeiramente, sabemos que $o \in \mathcal{B}_{i} \oplus \mathcal{B}_{i}^{t}, \forall 1 \leq i \leq N$, pela Prop. 2.1.6. Assim sendo, $\{o\} \subseteq \mathcal{B}_{i} \oplus \mathcal{B}_{i}^{t}$. Usando a propriedade crescente da dilatação, temos que $\mathcal{A}_{i-1} \oplus o \subseteq \mathcal{A}_{i-1} \oplus \mathcal{B}_{i} \oplus \mathcal{B}_{i}^{t}, \forall 2 \leq i \leq N$. Mas $\mathcal{A}_{i-1} \oplus o=\mathcal{A}_{i-1}$ e $\mathcal{A}_{i-1} \oplus \mathcal{B}_{i} \oplus \mathcal{B}_{i}^{t}=\mathcal{A}_{i} \Rightarrow \mathcal{A}_{i-1} \subseteq \mathcal{A}_{i}, \forall 2 \leq i \leq N$, como queríamos.

Além disso, também é possível provar que os elementos da sequência gerada $\mathbb{A}$ obedecem a propriedade simétrica:

Proposição 3.1.3. Para qualquer sequência gerada $\mathbb{A}=\left(\mathcal{A}_{1}, \ldots, \mathcal{A}_{N}\right)$ e qualquer $1 \leq i \leq N, \mathcal{A}_{i}$ é simétrico.

Demonstração. Vamos fazer a prova por indução. $\mathcal{A}_{1}$ é simétrico pela Prop. 2.1 .7 e, para todos os outros casos, vale que $\mathcal{A}_{i}=\mathcal{A}_{i-1} \oplus \mathcal{B}_{i} \oplus \mathcal{B}_{i}^{t}=\mathcal{A}_{i-1} \oplus\left(\mathcal{B}_{i} \oplus \mathcal{B}_{i}^{t}\right)$. Suponha por indução que $\mathcal{A}_{k}$ é simétrico para todo $k<i$ e vamos provar que $\mathcal{A}_{i}$ é simétrico. $\mathcal{B}_{i} \oplus \mathcal{B}_{i}^{t}$ é simétrico pela Prop. 2.1.7. Pela hipótese de indução, temos que $\mathcal{A}_{i-1}$ é simétrico e podemos aplicar a Prop. 2.1.8, o que implica que $\mathcal{A}_{i-1} \oplus\left(\mathcal{B}_{i} \oplus \mathcal{B}_{i}^{t}\right)=\mathcal{A}_{i}$ é simétrico.

Corolário 3.1.4. Dados uma sequência gerada $\mathbb{A}=\left(\mathcal{A}_{1}, \ldots, \mathcal{A}_{N}\right)$ e dois pixels $p \in \mathcal{D}$ e $q \in \mathcal{D}$, $p \in q \oplus \mathcal{A}_{i} \Leftrightarrow q \in p \oplus \mathcal{A}_{i}, \forall 1 \leq i \leq N$.

Para simplificação de notação das proposições seguintes, será conveniente definirmos um símbolo para a soma de EEs consecutivos. Assim, usaremos a notação $\mathcal{B}^{\Sigma}$ para representar a seguinte operação:

$$
\mathcal{B}_{i}^{\Sigma}=\mathcal{B}_{1} \oplus \mathcal{B}_{2} \oplus \ldots \oplus \mathcal{B}_{i}, 1 \leq i \leq N
$$

Fazendo uso da Eq. 3.1, é possível mostrar que qualquer EE $\mathcal{A}_{i}, 1 \leq 1 \leq N$ da sequência gerada $\mathbb{A}$ pode ser definida a partir de $\mathcal{B}_{i}^{\Sigma}$ :

Proposição 3.1.5. Dada uma sequência $\mathbb{A}=\left(\mathcal{A}_{1}, \ldots, \mathcal{A}_{N}\right)$ gerada a partir da sequência geradora $\mathbb{B}=\left(\mathcal{B}_{1}, \ldots, \mathcal{B}_{N}\right), \mathcal{A}_{i}=\mathcal{B}_{i}^{\Sigma} \oplus\left(\mathcal{B}_{i}^{\Sigma}\right)^{t}, \forall 1 \leq i \leq N$.

Demonstração. Vamos fazer a prova por indução.

Para $i=1$ :

$\mathcal{A}_{1}=\mathcal{B}_{1} \oplus \mathcal{B}_{1}^{t}=\mathcal{B}_{1}^{\Sigma} \oplus\left(\mathcal{B}_{1}^{\Sigma}\right)^{t}$. Logo, para o caso base, a proposição é válida.

Suponha agora que a proposição é válida para todos os índices menores que $i$ e vamos provar que ela continua válida para o índice $i$ :

$\mathcal{A}_{i}=\mathcal{A}_{i-1} \oplus \mathcal{B}_{i} \oplus \mathcal{B}_{i}^{t}$

$=\left(\mathcal{B}_{i-1}^{\Sigma} \oplus\left(\mathcal{B}_{i-1}^{\Sigma}\right)^{t}\right) \oplus \mathcal{B}_{i} \oplus \mathcal{B}_{i}^{t}$ (hipótese de indução)

$=\left(\mathcal{B}_{i-1}^{\Sigma} \oplus \mathcal{B}_{i}\right) \oplus\left(\left(\mathcal{B}_{i-1}^{\Sigma}\right)^{t} \oplus \mathcal{B}_{i}^{t}\right)$

$=\left(\mathcal{B}_{1} \oplus \ldots \oplus \mathcal{B}_{i-1} \oplus \mathcal{B}_{i}\right) \oplus\left(\mathcal{B}_{1}^{t} \oplus \ldots \oplus \mathcal{B}_{i-1}^{t} \oplus \mathcal{B}_{i}^{t}\right)$

$=\left(\mathcal{B}_{1} \oplus \ldots \oplus \mathcal{B}_{i-1} \oplus \mathcal{B}_{i}\right) \oplus\left(\mathcal{B}_{1} \oplus \ldots \oplus \mathcal{B}_{i-1} \oplus \mathcal{B}_{i}\right)^{t}$ (Cor. 2.1.3)

$=\mathcal{B}_{i}^{\Sigma} \oplus\left(\mathcal{B}_{i}^{\Sigma}\right)^{t}$. 
Representar os EEs $\mathcal{A}_{i}, 1 \leq i \leq N$ da sequência gerada $\mathbb{A}$ a partir da operação $\mathcal{B}_{i}^{\Sigma}$ é conveniente porque, como veremos na proposição seguinte, existe uma equivalência entre encontrar elementos vizinhos sob $\mathcal{A}_{i}$ e procurar por elementos nas quais suas vizinhanças sob $\mathcal{B}_{i}^{\Sigma}$ se intersectam:

Proposição 3.1.6. Dada uma sequência $\mathbb{A}=\left(\mathcal{A}_{1}, \ldots, \mathcal{A}_{N}\right)$ gerada a partir da sequência geradora $\mathbb{B}=\left(\mathcal{B}_{1}, \ldots, \mathcal{B}_{N}\right)$, dois pixels $p \in \mathcal{D}, q \in \mathcal{D}$ e um inteiro $i$ tal que $1 \leq i \leq N$, vale que $p \oplus \mathcal{B}_{i}^{\Sigma} \cap q \oplus$ $\mathcal{B}_{i}^{\Sigma} \neq \emptyset \Leftrightarrow p \in q \oplus \mathcal{A}_{i}$.

Demonstração. $p \oplus \mathcal{B}_{i}^{\Sigma} \cap q \oplus \mathcal{B}_{i}^{\Sigma} \neq \emptyset \Leftrightarrow$

$\exists r$ tal que $r \in p \oplus \mathcal{B}_{i}^{\Sigma}$ e $r \in q \oplus \mathcal{B}_{i}^{\Sigma} \Leftrightarrow$

$\exists r$ tal que $p \in r \oplus\left(\mathcal{B}_{i}^{\Sigma}\right)^{t}$ e $r \in q \oplus \mathcal{B}_{i}^{\Sigma}$ (Prop. 2.1.1)

$\Leftrightarrow p \in\left(q \oplus \mathcal{B}_{i}^{\Sigma}\right) \oplus\left(\mathcal{B}_{i}^{\Sigma}\right)^{t}$ (Prop. 2.1.4)

$\Leftrightarrow p \in q \oplus\left(\mathcal{B}_{i}^{\Sigma} \oplus\left(\mathcal{B}_{i}^{\Sigma}\right)^{t}\right)$ (associatividade)

$\Leftrightarrow p \in q \oplus \mathcal{A}_{i}$ (Prop. 3.1.5).

Portanto é possível encontrar todos os pares de pixels vizinhos sob qualquer vizinhança $\mathcal{A}_{i}$ de $\mathbb{A}$ nos restringindo aos EEs $\mathcal{B}_{i}^{\Sigma}$. O EE definido por $\mathcal{B}_{i}^{\Sigma}$ possui tamanho menor que $\mathcal{A}_{i}$ e pode ser rapidamente obtido a partir de $\mathcal{B}_{i-1}^{\Sigma}$, o que tornará o algoritmo de construção das árvores mais eficiente.

\subsection{Componentes conexos de um conjunto de nível}

Ao fazermos uso das sequências geradas $\mathbb{A}$ e geradoras $\mathbb{B}$, obtivemos uma propriedade que permite descobrir os elementos vizinhos sob qualquer $\mathcal{A}_{i} \in \mathbb{A}, 1 \leq i \leq N$, ao procurarmos por intersecções de vizinhanças de pares de pixels sob EEs da sequência geradora $\mathbb{B}$ (Prop. 3.1.6). Se tivermos o conjunto de todos os pares de pixels vizinhos de uma imagem, podemos fazer uso desta informação para obtermos os CCs da imagem sob $\mathcal{A}_{i}$, o que poderá ser usado posteriormente na construção das maxtrees. Portanto, a forma de se obter as interseç̧ões das vizinhanças de dois pixels impacta diretamente na complexidade do algoritmo final. Por esta razão, nesta seção, nos concentramos em uma forma eficiente de se obter as intersecções das vizinhanças.

\subsubsection{Rotulação: obtendo os componentes conexos das vizinhanças da sequência gerada $\mathbb{A}$}

Por simplicidade, vamos primeiro analisar como obtermos um algoritmo capaz de encontrar intersecções de vizinhanças em imagens binárias e depois partiremos ao caso geral.

Seja $X$ uma imagem binária. A fim de usar as propriedades de dilatação estabelecidas, faremos uso de uma função adicional, que servirá para rotular cada pixel $q \in \mathcal{D}$ com relação a todos os pixels de objeto $p$ de $X$ em que $q$ é vizinho de $p$ na vizinhança $\mathcal{A}=\mathcal{B} \oplus \mathcal{B}^{t}$. A esta função daremos o nome de $b \operatorname{Rot}(X, \mathcal{B})(b \operatorname{Rot}(X, \mathcal{B}): \mathcal{D} \rightarrow P(X)$, onde $P(X)$ é o conjunto das partes de $X$.) e a definiremos da seguinte maneira:

$$
[b \operatorname{Rot}(X, \mathcal{B})](q)=\{p \in X \mid q \in p \oplus \mathcal{B}\}
$$

Uma interpretação possível para a Equação 3.2 consiste do seguinte procedimento: para todos os pixels $p \in X$, verificamos quem são os vizinhos de $p$ sob $\mathcal{B}$ (lembrando que tais vizinhos são os elementos do conjunto $p \oplus \mathcal{B}$ ) e atribuímos a todos eles o rótulo $p$. Chamaremos esta atribuição de propagação do rótulo de $p$. Note que isso pode fazer com que um único pixel possua vários rótulos e, se for o caso, estes rótulos indicarão não apenas que houve uma intersecção naquele ponto, mas também indicarão quais são os pixels que se tornarão vizinhos considerando a vizinhança $\mathcal{A}=\mathcal{B} \oplus \mathcal{B}^{t}$, de acordo com a Prop. 3.1.6.

Para observar como é possível obter todos os pares de pixels vizinhos usando uma rotulação, considere o exemplo a seguir: 
Exemplo 3.2.1. Considere a imagem binária $X$ unidimensional apresentado na Fig. 3.2. $\mathcal{B}_{1}$ é o elemento estruturante apresentado abaixo, onde 'o' denota a origem. Suponha que queremos computar $\operatorname{RRot}\left(X, \mathcal{B}_{1}^{\Sigma}\right)$ para todos os pixels $a, b, c, d$, e.

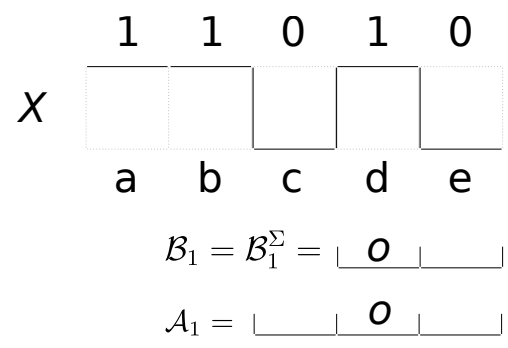

Figura 3.2: Imagem binária para fins de exemplo.

Para obtermos a rotulação de cada pixel, começamos com a função bRot $\left(X, \mathcal{B}_{1}^{\Sigma}\right)$ vazia e damos início à propagação dos rótulos de todos os $p \in X$ ( $a, b$ e d no exemplo).

Primeiramente, obtemos o conjunto de vizinhos de $a, a \oplus \mathcal{B}_{1}^{\Sigma}=\{a, b\}$. Assim, adicionamos o rótulo a a $\left[b \operatorname{Rot}\left(X, \mathcal{B}_{1}^{\Sigma}\right)\right](a)$ e a $\left[b \operatorname{Rot}\left(X, \mathcal{B}_{1}^{\Sigma}\right)\right](b)$.

A propagação dos outros rótulos consiste em continuar o processo para os pixels de objeto $b$ e d. Como $b \oplus \mathcal{B}_{1}^{\Sigma}=\{b, c\}$, o elemento $b$ é adicionado a $\left[b \operatorname{Rot}\left(X, \mathcal{B}_{1}^{\Sigma}\right)\right](b)$ e $\left[b \operatorname{Rot}\left(X, \mathcal{B}_{1}^{\Sigma}\right)\right](c)$. Analogamente, como $d \oplus \mathcal{B}_{1}^{\Sigma}=\{d, e\}$, d é adicionado a $\left[b \operatorname{Rot}\left(X, \mathcal{B}_{1}^{\Sigma}\right)\right](d)$ e $\left[b \operatorname{Rot}\left(X, \mathcal{B}_{1}^{\Sigma}\right)\right](e)$ e, após a propagação do rótulo $d$, obteremos o seguinte resultado.

- $\left[b \operatorname{Rot}\left(X, \mathcal{B}_{1}^{\Sigma}\right)\right](a)=\{a\} ;$

- $\left[b \operatorname{Rot}\left(X, \mathcal{B}_{1}^{\Sigma}\right)\right](b)=\{a, b\}$;

- $\left[b \operatorname{Rot}\left(X, \mathcal{B}_{1}^{\Sigma}\right)\right](c)=\{b\}$;

- $\left[b \operatorname{Rot}\left(X, \mathcal{B}_{1}^{\Sigma}\right)\right](d)=\{d\}$;

- $\left[b \operatorname{Rot}\left(X, \mathcal{B}_{1}^{\Sigma}\right)\right](e)=\{d\}$.

É possivel observar que a e b pertencem a $\left[\right.$ Rot $\left.\left(X, \mathcal{B}_{1}^{\Sigma}\right)\right](b)$, o que implica que a e $b$ serão vizinhos sob $\mathcal{A}=\mathcal{B} \oplus \mathcal{B}^{t}$.

Uma definição alternativa da Prop. 3.2, fazendo uso da Prop. 2.1.1, consiste da seguinte equação:

$$
[b \operatorname{Rot}(X, \mathcal{B})](q)=\left\{p \in X \mid p \in q \oplus \mathcal{B}^{t}\right\}
$$

Embora as duas definições sejam equivalentes, vamos preferir a Equação 3.2 porque será esta a definição que usaremos no algoritmo. Por outro lado, a definição obtida na Equação 3.3 é mais intuitiva.

Uma propriedade útil da função de rotulação é que, como $B_{i+1}^{\Sigma}=B_{i}^{\Sigma} \oplus \mathcal{B}_{i+1}$, a atualização de $b \operatorname{Rot}\left(X, \mathcal{B}_{i+1}^{\Sigma}\right)$ pode ser feita a partir de $b \operatorname{Rot}\left(X, \mathcal{B}_{i}^{\Sigma}\right)$ :

Proposição 3.2.2. Seja $\mathbb{B}=\left(\mathcal{B}_{1}, \ldots, \mathcal{B}_{N}\right)$ uma sequência geradora e $X$ uma imagem binária. Então, $\forall 1 \leq i<N,\left[b \operatorname{Rot}\left(X, \mathcal{B}_{i+1}^{\Sigma}\right)\right](q)=\bigcup_{q \in x \oplus \mathcal{B}_{i+1}}\left[b \operatorname{Rot}\left(X, \mathcal{B}_{i}^{\Sigma}\right)\right](x)=\bigcup_{x \in q \oplus \mathcal{B}_{i+1}^{t}}\left[b \operatorname{Rot}\left(X, \mathcal{B}_{i}^{\Sigma}\right)\right](x)$.

Demonstração. Vamos provar $\left[b \operatorname{Rot}\left(X, \mathcal{B}_{i+1}^{\Sigma}\right)\right](q)=\bigcup_{x \in q \oplus \mathcal{B}_{i+1}^{t}}\left[b \operatorname{Rot}\left(X, \mathcal{B}_{i}^{\Sigma}\right)\right](x)$.

Por definição, temos que

$\left[b \operatorname{Rot}\left(X, \mathcal{B}_{i+1}^{\Sigma}\right)\right](q)=\left\{p \in X \mid p \in q \oplus\left(\mathcal{B}_{i+1}^{\Sigma}\right)^{t}\right\}=\left\{p \in X \mid p \in q \oplus\left(\mathcal{B}_{i}^{\Sigma}\right)^{t} \oplus \mathcal{B}_{i+1}^{t}\right\}$.

Fixe um $p \in X$ qualquer do conjunto. Então, deve valer a seguinte propriedade:

$\exists b_{1} \in\left(\mathcal{B}_{j}^{\Sigma}\right)^{t}$ e $b_{2} \in \mathcal{B}_{i+1}^{t}$ tais que $p=q+b_{1}+b_{2}$

\footnotetext{
${ }^{1} \mathrm{O}$ símbolo $\square$ está sendo usado também para identificar o fim de um exemplo
} 
$\Leftrightarrow \exists b_{1} \in\left(\mathcal{B}_{i}^{\Sigma}\right)^{t}$ e $b_{2} \in \mathcal{B}_{i+1}^{t}$ tais que $p=x+b_{1}\left(x=q+b_{2}\right)$

$\Leftrightarrow p \in x \oplus\left(\mathcal{B}_{i}^{\Sigma}\right)^{t}$

$\Leftrightarrow p \in\left[b \operatorname{Rot}\left(X, \mathcal{B}_{i}^{\Sigma}\right)\right](x)$.

Como $x=q+b_{2}$, vale que $x \in q \oplus \mathcal{B}_{i+1}^{t}$ e, portanto,

$\left[b \operatorname{Rot}\left(X, \mathcal{B}_{i+1}^{\Sigma}\right)\right](q) \subseteq \bigcup_{x \in q \oplus \mathcal{B}_{i+1}^{t}}\left[b \operatorname{Rot}\left(X, \mathcal{B}_{i}^{\Sigma}\right)\right](x)$.

A prova da volta consiste em fazer o caminho inverso do descrito na prova.

Através da Prop. 3.2.2, sabemos que é possível obter $\left[b R o t\left(X, \mathcal{B}_{i+1}^{\Sigma}\right)\right](q)$ propagando o conjunto de rótulos de todos os pixels $x \in q \oplus \mathcal{B}_{i+1}^{t}$. Esta proposição será usada posteriormente na construção do algoritmo.

Uma vez mostrado que os rótulos podem ser obtidos de forma incremental considerando as rotulações dos passos anteriores, resta saber como usar a função para obtermos os CCs da imagem binária $X$ com relação aos EEs da sequência gerada $\mathbb{A}$. Para tanto, algumas propriedades extras precisam ser mostradas. Primeiro, vamos mostrar que dois pixels de objeto $p_{1}$ e $p_{2}$ de $X$ que pertencem a um mesmo $\left[b \operatorname{Rot}\left(X, \mathcal{B}_{i}^{\Sigma}\right)\right](q), q \in \mathcal{D}$, são vizinhos sob $\mathcal{A}_{i}$ :

Proposição 3.2.3. Seja $\mathbb{A}=\left(\mathcal{A}_{1}, \ldots, \mathcal{A}_{N}\right)$ a sequência gerada a partir da sequência geradora $\mathbb{B}=\left(\mathcal{B}_{1}, \ldots, \mathcal{B}_{N}\right)$ e $X$ uma imagem binária. Se $\mid\left[\left(\operatorname{bRot}\left(X, \mathcal{B}_{i}^{\Sigma}\right)\right](q) \mid>1\right.$ para algum $1 \leq i \leq N$ e $q \in \mathcal{D}$, então para todo par de pixels $\left(p_{1}, p_{2}\right) \operatorname{com} p_{1} \in\left[b \operatorname{Rot}\left(X, \mathcal{B}_{i}^{\Sigma}\right)\right](q)$ e $p_{2} \in\left[b \operatorname{Rot}\left(X, \mathcal{B}_{i}^{\Sigma}\right)\right](q)$, vale que $p_{2} \in p_{1} \oplus \mathcal{A}_{i}$ (ou $p_{1} \in p_{2} \oplus \mathcal{A}_{i}$, pois $\mathcal{A}_{i}$ é simétrico).

Demonstração. Como $p_{1} \in\left[b \operatorname{Rot}\left(X, \mathcal{B}_{i}^{\Sigma}\right)\right](q)$ e $p_{2} \in\left[b \operatorname{Rot}\left(X, \mathcal{B}_{i}^{\Sigma}\right)\right](q)$, então vale que $q \in p_{1} \oplus \mathcal{B}_{i}^{\Sigma}$ e $q \in p_{2} \oplus \mathcal{B}_{i}^{\Sigma} \Rightarrow p_{1} \oplus \mathcal{B}_{i}^{\Sigma} \cap p_{2} \oplus \mathcal{B}_{i}^{\Sigma} \neq \emptyset$. Agora, basta aplicar a Prop. 3.1.6.

Provado a Prop. 3.2.3, vamos estender a ideia para um par de pixels $p_{1}, p_{2} \in X$ conexos, primeiro mostrando para o caso particular em que só há um elemento intermediário e, depois, para o caso geral.

Proposição 3.2.4. Seja $\mathbb{A}=\left(\mathcal{A}_{1}, \ldots, \mathcal{A}_{N}\right)$ a sequência gerada a partir da sequência geradora $\mathbb{B}=\left(\mathcal{B}_{1}, \ldots, \mathcal{B}_{N}\right)$ e $X$ uma imagem binária. Dados dois pixels $q_{1} \in \mathcal{D}$ e $q_{2} \in \mathcal{D}$, se existe um pixel $r \in\left[b \operatorname{Rot}\left(X, \mathcal{B}_{i}^{\Sigma}\right)\right]\left(q_{1}\right) \cap\left[b \operatorname{Rot}\left(X, \mathcal{B}_{i}^{\Sigma}\right)\right]\left(q_{2}\right)$, então para todo $p_{1} \in\left[b \operatorname{Rot}\left(X, \mathcal{B}_{i}^{\Sigma}\right)\right]\left(q_{1}\right)$ e todo $p_{2} \in\left[\operatorname{bRot}\left(X, \mathcal{B}_{i}^{\Sigma}\right)\right]\left(q_{2}\right)$, vale que $p_{1}$ e $p_{2}$ pertencem ao mesmo $C C$ sob vizinhança $\mathcal{A}_{i}$.

Demonstração. Tome $p_{1} \in\left[b \operatorname{Rot}\left(X, \mathcal{B}_{i}^{\Sigma}\right)\right]\left(q_{1}\right)$ e $p_{2} \in\left[b \operatorname{Rot}\left(X, \mathcal{B}_{i}^{\Sigma}\right)\right]\left(q_{2}\right)$ quaisquer. Por definição, $p_{1}$, $p_{2}$ e $r$ são todos pixels de objeto. Pela Prop. 3.2.3, $p_{1} \in r \oplus \mathcal{A}_{i}$ e $r \in p_{2} \oplus \mathcal{A}_{i}$. Assim sendo, a sequência $\left(p_{1}, r, p_{2}\right)$ é um caminho em $X$ de $p_{1}$ a $p_{2}$ sob $\mathcal{A}_{i}$.

Proposição 3.2.5. Seja $\mathbb{A}=\left(\mathcal{A}_{1}, \ldots, \mathcal{A}_{N}\right)$ a sequência gerada a partir da sequência geradora $\mathbb{B}=$ $\left(\mathcal{B}_{1}, \ldots, \mathcal{B}_{N}\right)$ e $X$ uma imagem binária. Se existem pixels $\left\{q_{0}, q_{1}, \ldots, q_{N Q}\right\}$ e pixels $\left\{r_{1}, r_{2}, \ldots, r_{N Q}\right\}$ tais que $r_{k} \in\left[b \operatorname{Rot}\left(X, \mathcal{B}_{i}^{\Sigma}\right)\right]\left(q_{k-1}\right) \cap\left[b \operatorname{Rot}\left(X, \mathcal{B}_{i}^{\Sigma}\right)\right]\left(q_{k}\right), \forall 1 \leq k \leq N Q$, então para todo $p_{u} \in$ $\left[b \operatorname{Rot}\left(X, \mathcal{B}_{i}^{\Sigma}\right)\right]\left(q_{u}\right)$ e todo $p_{v} \in\left[b \operatorname{Rot}\left(X, \mathcal{B}_{i}^{\Sigma}\right)\right]\left(q_{v}\right), 0 \leq u<v \leq N Q$, $p_{u}$ e p p pertencem ao mesmo $C C$ sob vizinhança $\mathcal{A}_{i}$.

Demonstração. Tome $u$ e $v$ quaisquer satisfazendo $0 \leq u<v \leq N Q$ e tome $p_{u}$ e $p_{v}$ quaisquer satisfazendo $p_{u} \in\left[b \operatorname{Rot}\left(X, \mathcal{B}_{i}^{\Sigma}\right)\right]\left(q_{u}\right)$ e $p_{v} \in\left[b \operatorname{Rot}\left(X, \mathcal{B}_{i}^{\Sigma}\right)\right]\left(q_{v}\right) \cdot p_{u}$ e $p_{v}$ são pixels de objeto, e o mesmo vale para todos os $r_{w} \operatorname{com} u<w \leq v$.

Além disso, valem as seguintes 3 propriedades:

1. $r_{u+1} \in p_{u} \oplus B_{i}$, pois tanto $p_{u}$ quanto $r_{u+1}$ são elementos de $\left[b \operatorname{Rot}\left(X, \mathcal{B}_{i}^{\Sigma}\right)\right]\left(q_{u}\right)$ e vale a Prop. 3.2.3.

2. $r_{w+1} \in r_{w} \oplus B_{i}$ para todo $u<w<v$, pois tanto $r_{w+1}$ quanto $r_{w}$ são elementos de $\left[b \operatorname{Rot}\left(X, \mathcal{B}_{i}^{\Sigma}\right)\right]\left(q_{w}\right)$ e vale a Prop. 3.2.3.

3. $p_{v} \in r_{v} \oplus B_{i}$, pois tanto $p_{v}$ quanto $r_{v}$ são elementos de $\left[b \operatorname{Rot}\left(X, \mathcal{B}_{i}^{\Sigma}\right)\right]\left(q_{v}\right)$ e vale a Prop. 3.2.3.

Logo, a sequência $\left(p_{u}, r_{u+1}, \ldots, r_{v-1}, r_{v}, p_{v}\right)$ é um caminho de $p_{u}$ a $p_{v}$ em $X \operatorname{sob} \mathcal{A}_{i}$. 


\subsubsection{Grafo de rotulações}

Uma vez que dispomos de uma forma de se obter pixels vizinhos e conexos sob uma vizinhança $\mathcal{A}_{i} \in \mathbb{A}, 1 \leq i \leq N$ usando as funções de rotulação, é possível a extração dos CCs de qualquer imagem binária $X$. A fim de ilustrar o processo de rotulação e os CCs obtidos, vamos definir o grafo de rotulações, de $X$ sob a vizinhança $\mathcal{A}_{i}, G_{R o t}=\left(V_{R o t}, E_{R o t}\right)$ da seguinte forma:

Definição 3.2.6. O grafo de rotulações $G_{R o t}$ é definido como o par $G_{R o t}=\left(V_{R o t}, E_{R o t}\right)$ tal que:

1. $V_{\text {Rot }}=X$

2. Dados $p_{1} \in V_{R o t}$ e $p_{2} \in V_{R o t},\left\{p_{1}, p_{2}\right\} \in E_{R o t}$ se existe $q \in \mathcal{D}$ tal que $p_{1} \in\left[b \operatorname{Rot}\left(X, \mathcal{B}_{i}^{\Sigma}\right)\right](q)$ e $p_{2} \in\left[b \operatorname{Rot}\left(X, \mathcal{B}_{i}^{\Sigma}\right)\right](q)$.

Esta definição será usada apenas para ilustrar que a teoria desenvolvida nos permite obter, de fato, os CCs dos conjuntos de níveis que darão origem às árvores. Porém, em termos de implementação, o grafo de rotulação não será implementado.

Dito isso, vamos agora provar que os CCs o grafo de rotulações obtido a partir da função de rotulação $b \operatorname{Rot}\left(X, \mathcal{B}_{i}^{\Sigma}\right.$ consistem dos CCs de $X$ sob a vizinhança $\mathcal{A}_{i}$ :

Proposição 3.2.7. Dadas uma sequência gerada $\mathbb{A}$ e uma imagem binária $X$, os CCs do grafo de rotulações $G_{R o t}$ gerado a partir da Def. 3.2.6 consistem dos CCs de $X$ sob vizinhança $\mathcal{A}_{i}$.

Demonstração. Fixe um CC $\mathcal{C}_{R o t}$ qualquer de $G_{R o t}$. Primeiramente, vamos provar que existe um $\operatorname{CC~} \mathcal{C}_{\left(X, \mathcal{A}_{i}\right)}$ de $X$ sob vizinhança $\mathcal{A}_{i}$ satisfazendo $\mathcal{C}_{R o t} \subseteq \mathcal{C}_{\left(X, \mathcal{A}_{i}\right)}$.

Tome um par qualquer $\left(p_{1}, p_{2}\right)$ tal que $p_{1} \in \mathcal{C}_{R o t}$ e $p_{2} \in \mathcal{C}_{R o t}$. Temos que provar que tanto $p_{1}$ quanto $p_{2}$ pertencem a $\mathcal{C}_{\left(X, \mathcal{A}_{i}\right)}$ e existe caminho entre eles dentro do componente.

Como $p_{1} \in \mathcal{C}_{R o t}$ e $p_{2} \in \mathcal{C}_{R o t}$, existe um caminho de $p$ a $q, \pi\left(p_{1}, p_{2}\right)=\left(p_{1}, r_{1}, \ldots, r_{N R-1}, r_{N R}, p_{2}\right)$ no grafo $G_{R o t}$ com $r_{1}$ vizinho de $p_{1}, r_{k}$ vizinho de $r_{k-1}$ para todo $1<k \leq N R$ e $p_{2}$ vizinho de $r_{N R} . r_{k}$ é vizinho de $r_{k-1} \Rightarrow\left\{r_{k}, r_{k-1}\right\} \in E_{R o t}$. Assim, por definição, existe um conjunto $\left\{q_{0}, q_{1}, \ldots, q_{N R}\right\}$ tal que $r_{1}$ e $p_{1}$ estão em $\left[b \operatorname{Rot}\left(X, \mathcal{B}_{i}\right)\right]\left(q_{0}\right), p_{2}$ e $r_{N R}$ estão em $\left[b \operatorname{Rot}\left(X, \mathcal{B}_{i}\right)\right]\left(q_{N R}\right), r_{k}$ e $r_{k-1}$ estão em $\left[b \operatorname{Rot}\left(X, \mathcal{B}_{i}\right)\right]\left(q_{k-1}\right)$ para todo $1<k \leq N R$. Isso implica que $r_{k} \in\left[b \operatorname{Rot}\left(X, \mathcal{B}_{i}\right)\right]\left(q_{k}\right)$ e $r_{k} \in\left[b \operatorname{Rot}\left(X, \mathcal{B}_{i}\right)\right]\left(q_{k-1}\right)$ para $1 \leq k \leq N R$ e podemos aplicar a Prop. 3.2.5. Portanto, existe caminho de $p_{1}$ a $p_{2}$ sob vizinhança $\mathcal{A}_{i}$ e, consequentemente, $p_{1}$ e $p_{2}$ estão em um mesmo CC $\mathcal{C}_{\left(X, \mathcal{A}_{i}\right)}$ em $X$.

Isso prova que $\mathcal{C}_{\text {Rot }} \subseteq \mathcal{C}_{\left(X, \mathcal{A}_{i}\right)}$. A prova do caso $\mathcal{C}_{R o t} \supseteq \mathcal{C}_{\left(X, \mathcal{A}_{i}\right)}$ trata exatamente do caminho oposto feito nesta prova.

Exemplo 3.2.8. Partindo do exemplo da Fig. 3.2, o respectivo grafo de rotulações consiste do grafo mostrado na Fig. 3.3.

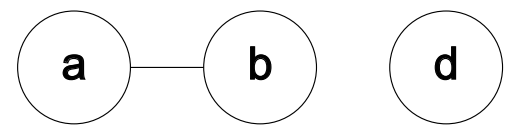

Figura 3.3: Grafo de rotulações $G_{R o t}$ de $X$ do exemplo anterior.

Isso implica que os $C C$ s de $X$ sob $\mathcal{A}_{1}$ são $\{a, b\}$ (pois a e b estão em $\left[b \operatorname{Rot}\left(X, \mathcal{B}_{1}^{\Sigma}\right)\right](b)$ ) e $\{d\}$.

\subsubsection{Rotulação em níveis de cinza}

O algoritmo de rotulação usado no caso binário não é tão eficiente quando aplicado diretamente em imagens em níveis de cinza: um mesmo pixel pode pertencer a vários CCs e teríamos que fazer a rotulação nível a nível.

Porém, as relações de inclusão dos CCs dos conjuntos de níveis nos permite inferir em quais níveis um determinado pixel é objeto ou fundo: é suficiente sabermos o nível de cinza dele. Afinal, dado um pixel $p$ de nível $f(p)$, para todo nível menor ou igual a $f(p), p$ é pixel de objeto. 
Assim sendo, para a rotulação em níveis de cinza, definiremos a função de rotulação Rot como:

$$
\left[\operatorname{Rot}\left(f, \mathcal{B}_{i}^{\Sigma}\right)\right](q)=\left\{(p, f(p)) \mid p \in \mathcal{D}, q \in p \oplus \mathcal{B}_{i}^{\Sigma}\right\}
$$

Podemos criar o grafo de rotulação de $f$ da mesma forma que fazíamos no grafo para as rotulações binárias. Neste caso, cada par $(p, f(p))$ se torna um vértice de $G_{R_{\text {ot }}(f)}$, onde $f(p)$ pode ser visto como o peso do vértice $p$. Se existe $q$ tal que $\left(p_{1}, f\left(p_{1}\right)\right)$ e $\left(p_{2}, f\left(p_{2}\right)\right)$ estão em $\left[\operatorname{Rot}\left(f, \mathcal{B}_{i}\right)\right](q)$, incluímos uma aresta entre seus respectivos vértices. A mesma ideia de usar um único grafo para todos os $i$ continua válida, uma vez que nenhuma aresta é removida devido à inclusão dos EEs da sequência $\mathbb{A}$. Para tanto, é suficiente usarmos pesos nas arestas representando o menor $i$ para o qual tal aresta foi adicionada, o que permite reconstruir os CCs não apenas para qualquer conjunto de nível $X_{\lambda}(f)$ como também para qualquer relação $\mathcal{A}_{i}$, bastando remover as arestas do grafo com peso maiores que $i$.

Exemplo 3.2.9. Considere a imagem $f$ unidimensional apresentada na Fig. 3.4. Queremos construir o grafo de rotulações de $f$ sob $\mathcal{A}_{1}$. Para tanto, repetimos o mesmo processo do exemplo anterior, com a diferença de que cada vértice do grafo agora possui também a informação do nível de cinza.

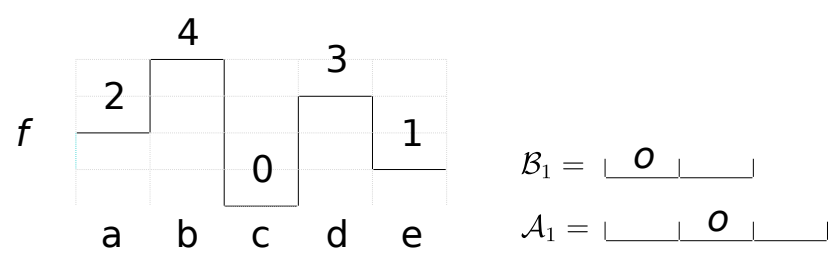

Figura 3.4: Imagem $f$ em níveis de cinza e uma vizinhança $\mathcal{A}_{1}$.

O resultado da rotulação é apresentada no lado esquerdo da Fig. 3.5, onde são mostrados os conjuntos de pixels pertencentes a cada $\left[\operatorname{Rot}\left(f, \mathcal{B}_{1}^{\Sigma}\right)\right](p), p \in\{a, b, c, d, e\}$. No lado direito é mostrado o grafo de rotulações gerado a partir da função de rotulação. Por exemplo, o resultado de $\left[\operatorname{Rot}\left(f, \mathcal{B}_{1}^{\Sigma}\right)\right](b)=\{(a, 2),(b, 4)\}$ (simplificado na figura apenas como $\{a, b\}$ dentro do retângulo laranja). Isso implica que há uma aresta entre $(a, 2)$ e $(b, 4)$ no grafo de rotulações (a aresta laranja no lado direito da figura). Ao final do processo de rotulação, teremos o grafo apresentado na direita, com cada cor representado uma intersecção de vizinhanças de pixels. O número 1 usado em cada aresta se refere ao indice $i$ em que a aresta foi adicionada.

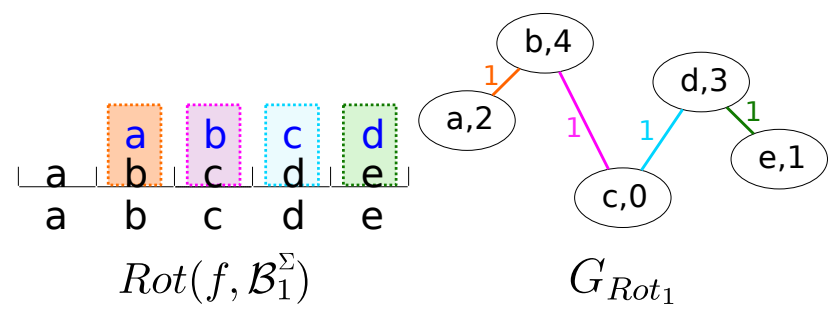

Figura 3.5: Grafo de rotulações $G_{\text {Rot }}$ da imagem $f$ mostrada na Fig. 3.4.

Note que a definição nos permite reconstruir as rotulações de cada conjunto de nível da seguinte forma:

$$
\left.\left[b \operatorname{Rot}\left(X_{\lambda}, \mathcal{B}_{i}\right)\right](q)=\left\{p \mid(p, f(p)) \in \operatorname{Rot}\left(f, \mathcal{B}_{i}\right)\right](q) \text { e } f(p) \geq \lambda\right\}
$$

A implicação da propriedade anterior é que podemos reconstruir os CCs de qualquer conjunto de nível $i$ usando o grafo de rotulações da imagem em níveis de cinza: basta gerar um subgrafo que consiste do grafo original sem os vértices com nível de cinza menor que $i$ (e sem as arestas que incidiam neles). 
Exemplo 3.2.10. Suponha que queremos reconstruir o conjunto de nível $X_{2}$ (isto é, a imagem binária que contém apenas pixels de nível de cinza maior ou igual a 2 como objetos) de $f$. Para tanto, basta remover os vértices que têm nivel de cinza menor que 2 e todas as arestas que incidem neles. O resultado pode ser visto na Fig. 3.6. Note que o resultado é igual aos CCs obtidos no exemplo binário, já que $X_{2}(f)$ é exatamente igual ao $X$ no exemplo binário, e a vizinhança $\mathcal{A}_{1}$ usada é a mesma.
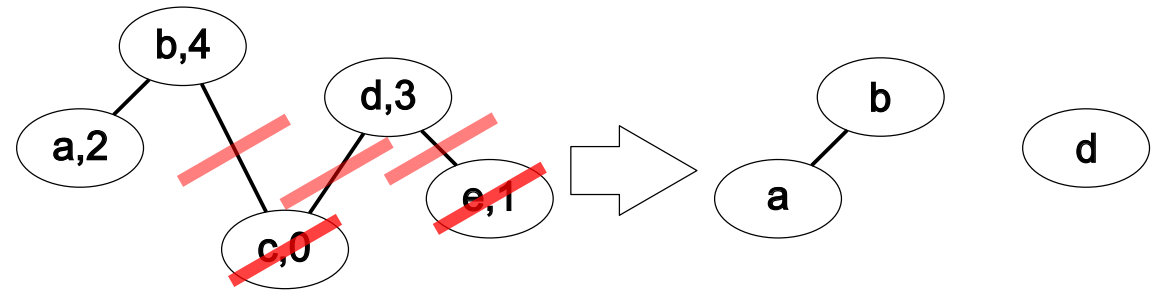

Figura 3.6: Extraindo CCs a partir do grafo de rotulações em niveis de cinza.

\subsubsection{Alteração de vizinhança}

As definições anteriores são feitas de forma a tornar eficiente a troca de vizinhança, isto é, obter os CCs de uma imagem $f$ sob $\mathcal{A}_{i+1}$ tendo os CCs de $f$ sob $\mathcal{A}_{i}$.

Suponha que temos os CCs de $f$ sob $\mathcal{A}_{i}$ e queremos os CCs de $f$ sob $\mathcal{A}_{i+1}$. Para encontrarmos os CCs sob $\mathcal{A}_{i+1}$, precisamos montar o grafo de rotulações e, para isso, precisamos rotular os pixels de acordo com as definições apresentadas.

Graças à definição da função Rot, podemos reaproveitar a computação de funções de índices $i$ anteriores para a computação de $\operatorname{Rot}_{i+1}$, através da seguinte definição:

Proposição 3.2.11. Dadas uma imagem em níveis de cinza $f$ e uma sequência geradora $\mathbb{B}=$ $\left(\mathcal{B}_{1}, \ldots, \mathcal{B}_{N}\right)$, vale que, para qualquer $1 \leq i<N$,

$$
\left[\operatorname{Rot}\left(f, \mathcal{B}_{i+1}^{\Sigma}\right)\right](q)=\bigcup_{q \in x \oplus \mathcal{B}_{i+1}^{\Sigma}}\left[\operatorname{Rot}\left(f, \mathcal{B}_{i}^{\Sigma}\right)\right](x)=\bigcup_{x \in q \oplus \mathcal{B}_{i+1}^{t}}\left[\operatorname{Rot}\left(f, \mathcal{B}_{i}^{\Sigma}\right)\right](x) .
$$

A definição é análoga à Prop. 3.2.2, apenas modificada para níveis de cinza. A prova da definição é estritamente semelhante à prova da Prop. 3.2.2.

Então, se encontrarmos os CCs de cada vizinhança de forma consecutiva, podemos aproveitar a função Rot calculada para o índice anterior. Obviamente, a função de rotulação não está calculada para o primeiro índice $(i=1)$ mas Rot para $i=1$ pode ser facilmente obtida usando a definição.

Além disso, graças à relação de inclusão das vizinhanças de $\mathbb{A}$, qualquer par de pixels $(p, q)$ que já estavam conectados por um caminho em $f$ sob $\mathcal{A}_{i}$ continuarão conectados pelo mesmo caminho sob $\mathcal{A}_{i+1}$, ou seja, uma aresta nunca será removida do grafo $G_{R o t}$ na mudança de índice de $i$ para $i+1$.

Em particular, vale a seguinte propriedade com relação aos CCs sob $\mathcal{A}_{i}$ e $\mathcal{A}_{i+1}$ :

Proposição 3.2.12. Para qualquer sequência gerada $\mathbb{A}=\left(\mathcal{A}_{1}, \ldots, \mathcal{A}_{N}\right)$ e qualquer imagem em níveis de cinza $f$, todo $C C$ de $f$ sob $\mathcal{A}_{i+1}$ é uma união de um ou mais $C C s$ de $f$ sob $\mathcal{A}_{i}, 1 \leq i<N$.

Demonstração. Como arestas podem ser apenas adicionadas ao grafo $G_{R o t}$, qualquer aresta adicionada ao conjunto ou liga dois vértices que pertencem ao mesmo componente, o que não altera o conjunto de CCs, ou liga dois vértices de dois componentes $\mathcal{C}_{1}$ e $\mathcal{C}_{2}$ distintos, o que faz com que exista caminho entre qualquer par de pixels $(p, q) \operatorname{com} p$ e $q$ em $\mathcal{C}_{1} \cup \mathcal{C}_{2}$, ou seja, $\mathcal{C}_{1} \cup \mathcal{C}_{2}$ se torna um único componente do grafo.

Tal fato vale para qualquer aresta adicionada e, como a ordem das adições de arestas é irrelevante (pois a união é associativa), cada $\mathrm{CC}$ do passo $i$, ao final da rotulação sob $\mathcal{B}_{i+1}^{\Sigma}$ pode ou ser igual a um CC do grafo no passo anterior $i$ (se nenhuma aresta incidindo a um vértice do CC foi adicionada) 
ou, se houve alguma alteração, consistir de um conjunto que é uma união de CCs do grafo criado a partir de $\mathcal{B}_{i}^{\Sigma}$.

Como nunca ocorre uma remoção de arestas, saber o primeiro índice $i$ no qual a aresta é adicionada é suficiente para determinar o menor $\mathcal{A}_{i}$ para o qual os respectivos vértices (pixels) se tornam vizinhos. Assim, podemos representar $G_{\text {Rot }}$ de forma geral para todos os $i$ usando um único grafo bastando adicionarmos pesos nas arestas. No caso, o peso usado consiste do menor $i$ no qual a aresta é adicionada e, se quisermos encontrar os CCs de uma certa vizinhança $\mathcal{A}_{i}$, basta remover todas as arestas de peso maior que $i$.

Exemplo 3.2.13. Vamos usar o mesmo $f$ do exemplo anterior, desta vez construindo os CCs também para a vizinhança $\mathcal{A}_{2}$ mostrada na Fig. 3.7.

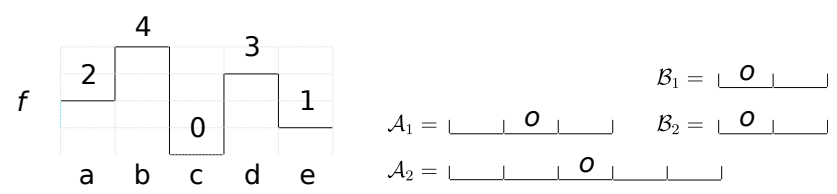

Figura 3.7: Imagem $f$ e algumas vizinhanças de $\mathbb{A}$.

Como sabemos, podemos usar a computação dos rótulos do passo 1 para computar os rótulos do passo 2. Afinal, $\left[\operatorname{Rot}\left(f, \mathcal{B}_{2}^{\Sigma}\right)\right](q)=\bigcup_{x \in q \oplus \mathcal{B}_{2}^{t}}\left[\operatorname{Rot}\left(f, \mathcal{B}_{1}^{\Sigma}\right)\right](x)$.

Por exemplo, $c \oplus \mathcal{B}_{2}^{t}=\{b, c\}$. Logo, $\left[\operatorname{Rot}\left(f, \mathcal{B}_{2}^{\Sigma}\right)\right](c)=\left[\operatorname{Rot}\left(f, \mathcal{B}_{1}^{\Sigma}\right)\right](b) \cup\left[\operatorname{Rot}\left(f, \mathcal{B}_{1}^{\Sigma}\right)\right](c)=$ $\{(a, 2),(b, 4)\} \cup\{(b, 4),(c, 0)\}=\{(a, 2),(b, 4),(c, 0)\}$, como mostrado na Fig. 3.8, simplificado como apenas $\{a, b, c\}$. Usando a ideia de propagação, esta atualização ocorreria ao propagarmos o rótulo de $b$ e $c$ e, a partir de então, $\left[\operatorname{Rot}\left(f, \mathcal{B}_{2}^{\Sigma}\right)\right](c)$ estaria atualizado.

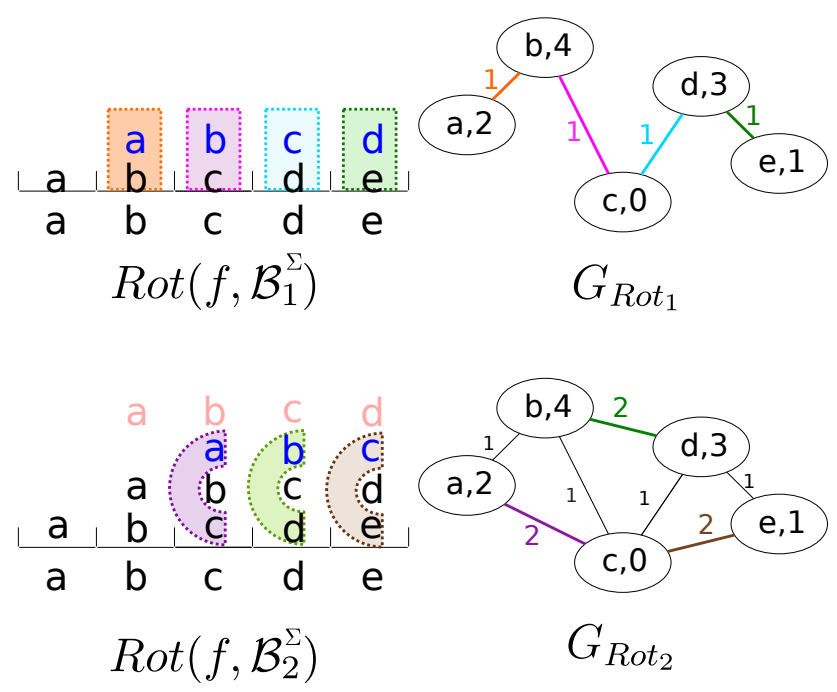

Figura 3.8: Rotulação de $f$ para as vizinhanças da imagem 3.7.

Ao lado esquerdo da figura, rótulos na cor preta indicam rótulos $p$ que já faziam parte de $\left[\operatorname{Rot}\left(f, \mathcal{B}_{1}^{\Sigma}\right)\right](q)$. Se um rótulo $p$ só foi adicionado no passo $i=2$, o rótulo está marcado em azul. Qualquer rótulo repetido na imagem está na cor vermelha. Cada nova aresta adicionada está representada em uma cor, onde os valores das arestas mostram em qual valor $i$ a aresta foi adicionada.

Assim como antes, é possivel reconstruir qualquer conjunto de nivel para qualquer passo $i$ já calculado. A Fig. 3.9 mostra exemplos para os CCs de $X_{2}$ sob $\mathcal{A}_{1}$ e $X_{1}$ sob $\mathcal{A}_{2}$. Novamente, $X_{2}$ sob $\mathcal{A}_{1}$ são os mesmos $C C s$ do exemplo binário, enquanto que $X_{1}$ sob $\mathcal{A}_{2}$ mostra o impacto de usarmos a vizinhança $\mathcal{A}_{2}$. Se não fosse por isso, não haveria aresta entre b e d e teríamos dois CCs ao invés de um único. Portanto, este $C C$ sob $\mathcal{A}_{2}$ consiste da união de dois CCs sob $\mathcal{A}_{1}:\{a, b\}$ com $\{d, e\}$. 


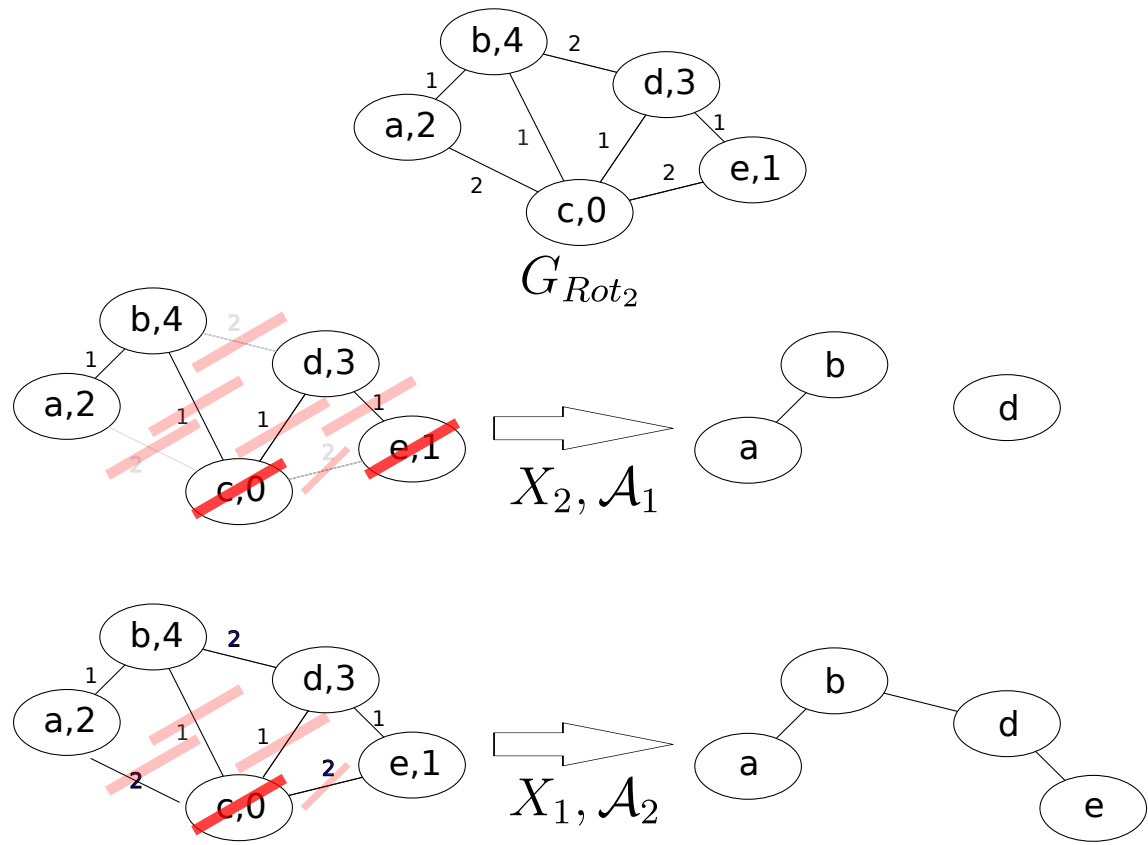

Figura 3.9: Obtendo CCs a partir do grafo de rotulações. 


\section{Capítulo 4}

\section{Implementação}

Neste capítulo, faremos uma revisão dos algoritmos já existentes para a construção da max-tree. A seguir, veremos como adaptar este algoritmo para o nosso problema, mostrando como a função de rotulação apresentada no Capítulo 3 pode ser eficientemente implementada para gerar a sequência de árvores restritas às vizinhanças da sequência gerada $\mathbb{A}$.

\subsection{Algoritmo de construção de max-trees}

Antes de descrevermos os algoritmo, é necessário descrevermos a estrutura de dados utilizada para o armazenamento da árvore. É conhecido [NC06, $\left.\mathrm{BGL}^{+} 07, \mathrm{WGH}^{+} 08\right]$ que se pode armazenar uma max-tree eficientemente usando uma estrutura de conjuntos disjuntos (union-find) [Tar75].

\subsubsection{Estrutura de conjuntos disjuntos}

Na estrutura de conjuntos disjuntos, cada elemento consiste em um identificador e um apontador para outro elemento do mesmo conjunto. Todos os elementos de um mesmo conjunto apontam em direção a um mesmo elemento, que será chamado de representante ou elemento canônico. O representante de um conjunto aponta para si mesmo.

A Fig. 4.1 apresenta um exemplo de uma estrutura de conjuntos disjuntos. Na figura, as letras são os identificadores e as setam indicam os apontadores. Elementos que apontam para si mesmo são os representantes $(a, d, e, i)$. Elementos que pertencem ao mesmo conjunto estão com a mesma cor.

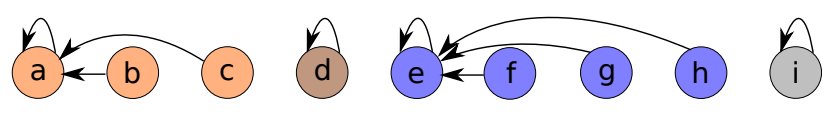

Figura 4.1: Representação gráfica da estrutura de conjuntos disjuntos.

Esta estrutura tem duas características úteis para o nosso problema: primeiro, ela permite saber quando dois elementos pertencem ou não a um mesmo conjunto (operação find) simplesmente procurando saber se os dois elementos têm o mesmo representante. Além disso, ela também permite realizar união de conjuntos de forma eficiente: para unir dois conjuntos disjuntos $C_{r}$ e $C_{s}$, cujos representantes são, respectivamente, $r$ e $s$, pode-se fazer a união destes conjuntos simplesmente adicionando um apontador de um representante ao outro (por exemplo, fazendo com que $r$ aponte para $s$ na estrutura).

Para uma melhor eficiência na implementação, geralmente faz-se com que o representante do conjunto com menor número de elementos aponte para o representante do conjunto maior.

Uma implementação da estrutura de conjuntos disjuntos é apresentada nos Algoritmos 1, 2 e 3. 

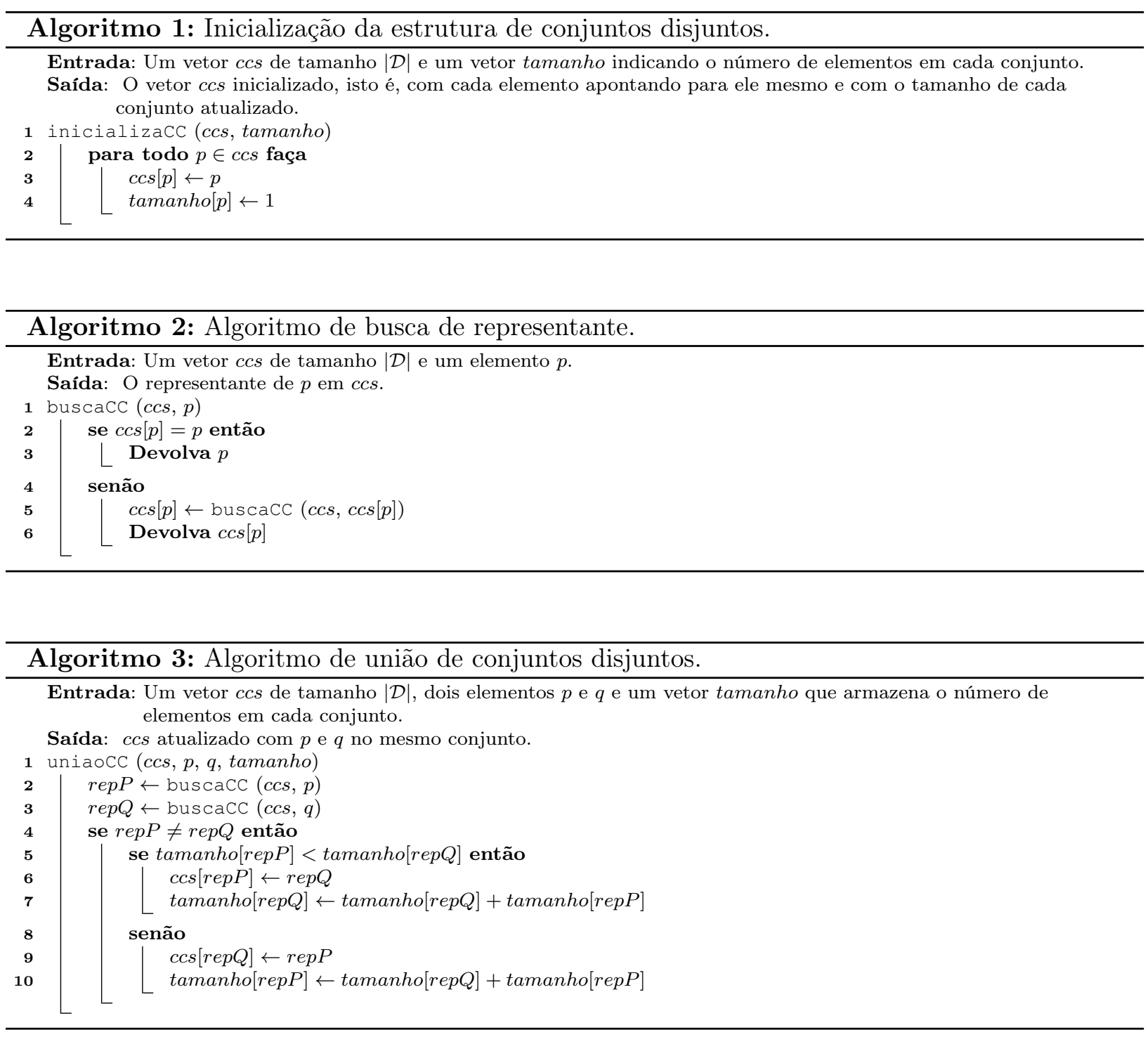

Para adaptar esta estrutura ao nosso problema, é necessário fazer uma pequena alteração, pois queremos usá-la para armazenar a max-tree. Assim, adicionaremos apontadores entre os representantes a fim de representar também relações de inclusão entre os conjuntos.

A estrutura modificada então satisfará a seguinte definição:

Definição 4.1.1. Considere que estamos usando a estrutura de union-find para representar a maxtree de um imagem $f$ sob uma vizinhança $\mathcal{A}$. Suponha que dois elementos $p, q \in \mathcal{D}$ são tais que $p$ aponta para $q$ na estrutura. Então, uma das afirmativas é verdadeira:

- $p$ e $q$ pertencem ao mesmo nó da max-tree e, neste caso, vale necessariamente que $f(p)=f(q)$;

- q representa o nó que é pai do nó que contém $p$ e, neste caso, vale necessariamente que $f(p)>f(q)$.

Usando a Definição 4.1.1, é possível obtermos todos os nós da árvore e as relações de parentesco entre eles apenas tendo esta estrutura e comparando os níveis de cinza de cada pixel. Assim, a condição da operação de busca de representante deve ser alterada, a fim de também levar em conta o nível de cinza. O algoritmo modificado é apresentado no Algoritmo 4.

É importante notar que a atribuição da linha 5 nos Algoritmo 4 não é necessária, sendo que podíamos simplesmente devolver o valor de representante (ccs, ccs $[p], f)$. Esta atribuição consiste de uma otimização conhecida como compressão de caminhos, que faz com que as operações posteriores de busca sejam mais rápidas. 


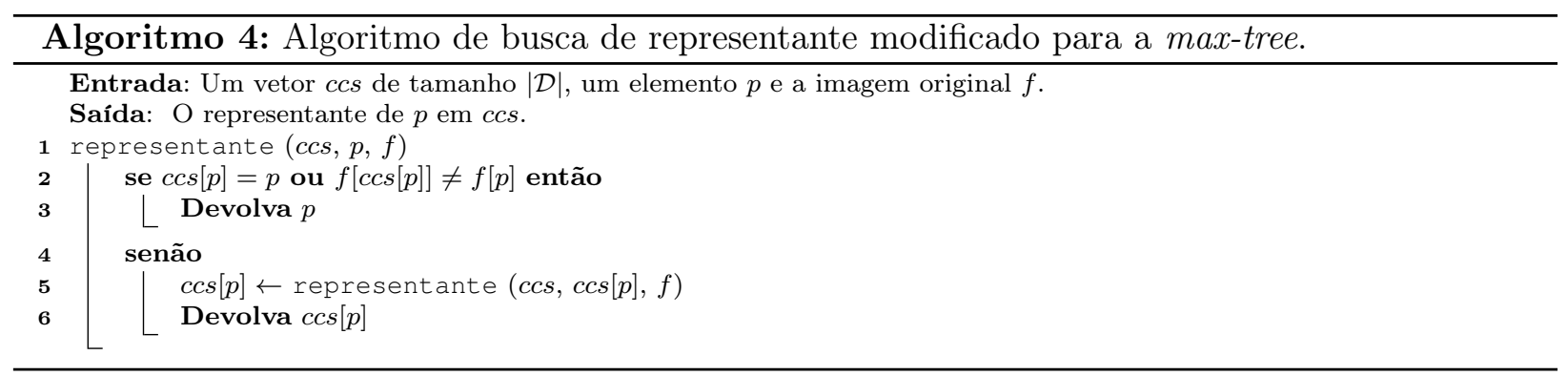

Podemos armazenar a estrutura em memória na forma de um vetor. Neste trabalho, usaremos um vetor de inteiros denominado pais (cada pixel $p$ é identificado univocamente por um número inteiro).

O único porém do uso deste vetor consiste do fato de que os nós apontam para os nós pai, sendo que um pós processamento é necessário se quisermos uma estrutura em forma de árvore que parta do nó raiz para os nós folha.

As relações de inclusão entre os conjuntos serão adicionados em algoritmo à parte, que consiste do algoritmo principal da construção da árvore. No Algoritmo 5 é mostrada a implementação do algoritmo de construção de Berger et al. [BGL $\left.{ }^{+} 07\right]$.

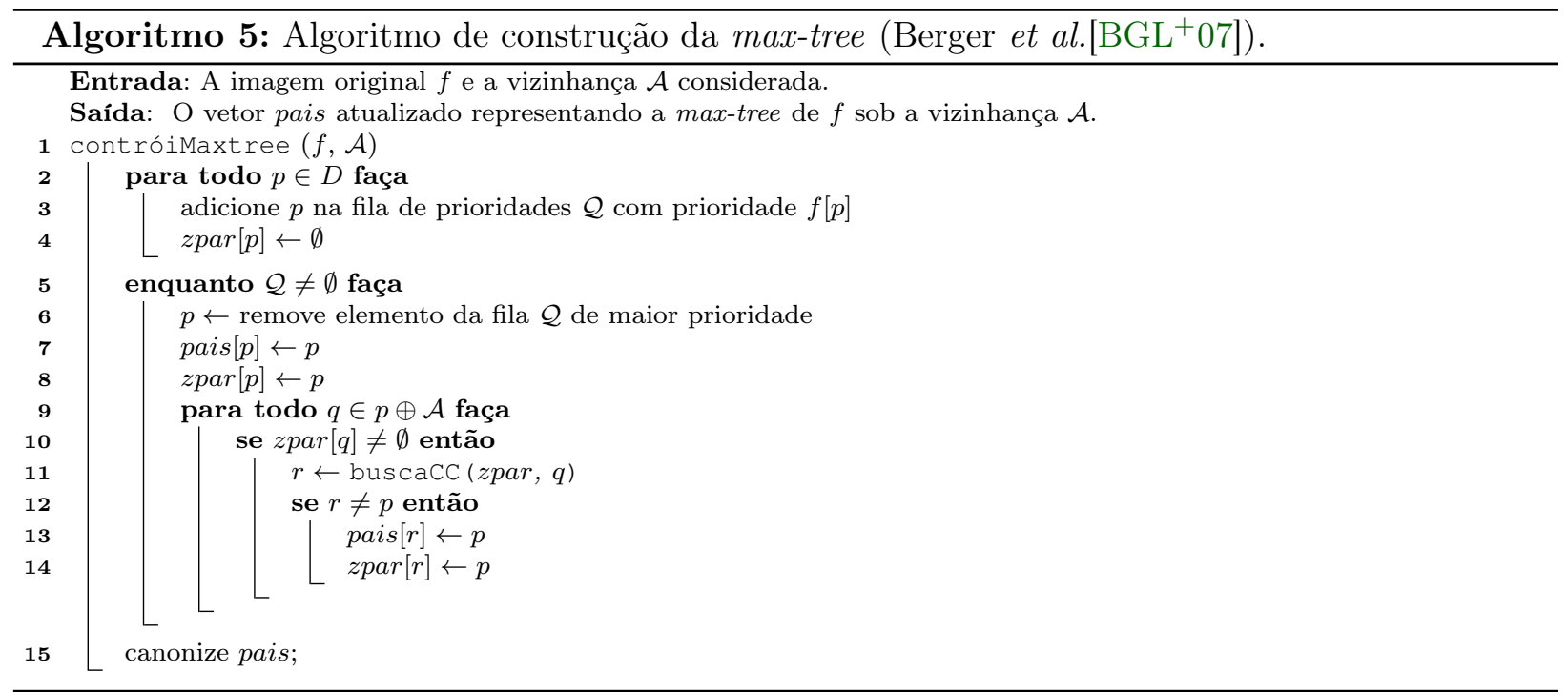

A operação de canonização consiste em comprimir o caminho de todos os elementos ao seu representante. Este assunto será retomado posteriormente na Seção 4.4.7.

\subsection{Computação eficiente dos rótulos (caso binário)}

O Algoritmo 5 servirá de base para o desenvolvimento do nosso algoritmo. Porém, antes de desenvolvermos um algoritmo eficiente para o caso em níveis de cinza, nos restringiremos ao caso binário para fins didáticos, visto que é um caso bem mais simples de ser analisado e muitas das ideias aqui apresentadas podem ser aproveitadas no caso em níveis de cinza.

A principal diferença no nosso algoritmo consiste na forma de encontrar os elementos vizinhos do pixel sendo processado no momento. Ao invés de fazermos isso diretamente, como era feito na linha 9 do Algoritmo 5, usaremos a função de rotulação.

Toda a teoria vista até agora nos induz a um algoritmo: pela Prop. 3.2.2, dado um pixel $q$ e fixado um conjunto de nível $X$, é possível atualizar $\left[b \operatorname{Rot}\left(X, \mathcal{B}_{i}^{\Sigma}\right)\right](q)$ usando $\left[b \operatorname{Rot}\left(X, \mathcal{B}_{i-1}^{\Sigma}\right)\right](q)$. Assim, é possível construir os CCs de forma incremental: construímos primeiro o conjunto de CCs 
sob $\mathcal{A}_{1}$ usando a definição de $b R$ ot para $i=1$, depois construímos o conjunto de CCs sob $\mathcal{A}_{2}$ usando o fato que $b \operatorname{Rot}\left(X, \mathcal{B}_{2}^{\Sigma}\right)$ pode ser facilmente obtido usando $b \operatorname{Rot}\left(X, \mathcal{B}_{1}^{\Sigma}\right)$ e assim por diante.

Porém, o algoritmo obtido diretamente da teoria vista pode ser melhorado em alguns pontos, resultando em um melhor desempenho com relação tanto a consumo de tempo quanto a consumo de memória, como veremos nas seções a seguir.

\subsubsection{Armazenamento eficiente da função de rotulação}

Um problema da função de rotulação é que o número de elementos para cada $\left[b \operatorname{Rot}\left(X, \mathcal{B}_{i}^{\Sigma}\right)\right](q)$ pode aumentar de acordo com o valor de $i$. Assim sendo, o uso desta função sem alterações pode acarretar em consumo excessivo de memória.

A função $b \operatorname{Rot}\left(X, \mathcal{B}_{i}^{\Sigma}\right)$ foi definida de forma a encontrar pixels de objeto vizinhos sob $\mathcal{A}_{i}$, o que é refletido na forma de arestas em $G_{\text {Rot }}$. Porém, como estamos interessados apenas em CCs, não é necessário saber se dois pixels são vizinhos, apenas se estão ou não conectados por algum caminho dentro de $X \operatorname{sob} \mathcal{A}_{i}$.

Em particular sabemos que, uma vez adicionada uma aresta, ela nunca mais será removida devido à inclusão das vizinhanças. Portanto, uma vez que dois vértices, digamos $p_{1}$ e $p_{2}$, são ligados por uma aresta, estes dois vértices pertencerão ao mesmo CC para qualquer passo $i$ posterior. Qualquer novo vértice $p_{3}$ que se una a um desses dois vértices estará automaticamente conectado por um caminho ao outro vértice, gerando um $\mathrm{CC}$ contendo $p_{1}, p_{2}$ e $p_{3}$. Analogamente, quando outros pixels se tornarem vizinhos de algum destes elementos do CC, a qualquer um dos elementos que estes novos pixels se conectem, eles estarão automaticamente ligado por um caminho aos outros elementos do componente.

Em outras palavras, podemos usar a estrutura de conjuntos disjuntos para representar os CCs. Dessa forma, ao invés de mantermos todos os pixels dentro de $\left[b \operatorname{Rot}\left(X, \mathcal{B}_{i}^{\Sigma}\right)\right](q)$, basta mantermos apenas o representante do conjunto. Assim, em termos de estrutura de dados, podemos usar uma função otimizada $b R t: \mathcal{D} \rightarrow \mathcal{D}$ que armazena apenas um pixel para cada posição, definido da seguinte forma:

Definição 4.2.1. Seja $\mathbb{B}=\left(\mathcal{B}_{1}, \ldots, \mathcal{B}_{N}\right)$ uma sequência geradora e $X$ uma imagem binária. Fixe $q \in \mathcal{D}$ e $1 \leq i \leq N$ e seja $r$ um vértice de $G_{R o t}$ que representa o CC em que os elementos de $\left[b \operatorname{Rot}\left(X, \mathcal{B}_{i}^{\Sigma}\right)\right](q)$ estão contidos ( $r$ pode ser qualquer elemento dentro do CC, desde que este CC sempre esteja representado por este mesmo $r)$. Então, a $\left[b R t\left(X, \mathcal{B}_{i}^{\Sigma}\right)\right](q)$ é simplesmente atribuído o elemento $r$, ou seja, $\left[b R t\left(X, \mathcal{B}_{i}^{\Sigma}\right)\right](q) \leftarrow r$.

\subsubsection{Detecção de rótulos inalterados}

A partir da teoria vista na seção anterior, elementos dentro da função de rotulação binária otimizada bRt agora representam um rótulo que indicam em qual CC tal rótulo está contido. Além disso, usar $b R t$ mantém os mesmos CCs da função $b R o t$, fazendo com que possamos fazer a propagação dos rótulos usando $b R t$ ao invés de $b R o t$.

Isso faz com que a seguinte propriedade seja válida:

Proposição 4.2.2. Tome $i$ e $j$ satisfazendo $1 \leq i<j \leq N$. Suponha que $\mathcal{B}_{i} \supseteq \mathcal{B}_{j}$ e tome um $q \in \mathcal{D}$ qualquer. Se $\left[b \operatorname{Rt}\left(X, \mathcal{B}_{i-1}^{\Sigma}\right)\right](q)$ for não-vazio, então a propagação do rótulo de $q$ no passo $j$ não adicionará nenhum vértice ao CC em que q está contido.

Demonstração. Como $\left[b R t\left(X, \mathcal{B}_{i-1}^{\Sigma}\right)\right](q) \neq \emptyset$, ao propagar o rótulo de $q$ no passo $i$, todos os elementos dentro de $q \oplus \mathcal{B}_{i}$ se tornam conexos ao CC em que $q$ pertence.

Quando propagarmos o rótulo de $q$ no passo $j$, faremos com que os vizinhos de $q$ sob $\mathcal{B}_{j}$ se conectem ao CC de $q$. Mas como $\mathcal{B}_{i} \supseteq \mathcal{B}_{j}$, tais vizinhos estavam todos contidos em $q \oplus \mathcal{B}_{i}$, ou seja, eles já estão foram conectados ao CC que contém $q$ no passo $i$.

A Prop. 4.2.2 pode ser vista em outros algoritmos da literatura: o que estamos essencialmente fazendo é uma dilatação em $X$ restrita aos EEs da sequência geradora $\mathbb{B}$. Se assumirmos que todos 
os EEs de $\mathbb{B}$ são iguais e tivermos, por exemplo, computado $X \oplus \mathcal{B}_{1}$, não é necessário dilatar todos os pixels da imagem para obter $X \oplus \mathcal{B}_{2}$, pois sabemos que os únicos pixels que podem modificar a imagem são aqueles que estão nas bordas ou, em outras palavras, os pixels presentes em $\left(X \oplus \mathcal{B}_{1}\right) \backslash X$, o que nos permite computar a dilatação de forma mais eficiente.

Esta propriedade permite evitar a propagação de rótulos desnecessários e ajuda a diminuir o consumo de tempo, mas pode ser custoso manter a informação de quais elementos estruturante foram usados para propagar cada pixel.

Porém, dependendo da escolha dos elementos estruturantes, é fácil prever se um elemento estruturante é repetido ou não. Um caso particular consiste em quando $\mathbb{B}$ é cíclico, isto é, existe um inteiro $c>0$ tal que $\mathcal{B}_{i}=\mathcal{B}_{i+c}$ para qualquer $1 \leq i \leq N-c$. Neste caso, basta propagar o rótulo de cada pixel por um ciclo. Saber quando um ciclo se encerrou para cada pixel também é simples: basta manter um contador para cada um até atingirmos o valor $c$. Por exemplo, no caso em que todos os elementos de $\mathbb{B}$ são iguais, o ciclo tem tamanho 1 , pois $\mathcal{B}_{i}=\mathcal{B}_{i+1}, 1 \leq i \leq N-1$. Além disso, a suposição de ciclo em $\mathbb{B}$ pode fazer sentido em muitas aplicações: como visto na Fig. 3.1, é possível gerar vizinhanças quadradas ou em linha usando uma sequência geradora $\mathbb{B}$ com ciclo 1 .

Se valer a propriedade cíclica da sequência $\mathbb{B}$, o algoritmo se torna ainda mais eficiente, pois podemos parar de propagar alguns rótulos quando eles atingem um ciclo completo.

No caso cíclico, uma outra vantagem consiste em saber quando o algoritmo converge: se após um ciclo completo nenhuma união ocorre na estrutura de conjuntos disjuntos e vale que $b R t\left(X, \mathcal{B}_{i}^{\Sigma}\right)=$ $b R t\left(X, \mathcal{B}_{i-1}^{\Sigma}\right)$, então sabemos que não há mais nenhuma união a ser feita e podemos parar de executar o algoritmo, pois continuar a execução não pode mais alterar os CCs.

\subsubsection{Algoritmo}

Com base em tudo isso, é possível elaborar uma forma eficiente de computar os CCs de cada EE em $\mathbb{A}$ usando os rótulos de cada pixel de objeto para um conjunto de nível. O algoritmo se baseará nas seguintes estruturas de dados e é apresentado abaixo (Algoritmo 6):

- int rotulo[] com tamanho $|\mathcal{D}|$. É a função $b R t$ apresentada anteriormente.

- Unionfind ccs com $|X|$ elementos. É uma estrutura que armazenará as uniões de pixels de $X$ quando eles se tornam vizinhos;

- Fila $\mathcal{Q}$ para organizar em ordem os pixels que devem ser processados;

- boolean adicionaFila[] com tamanho $|\mathcal{D}|$. Este vetor indica se o pixel $p$ deve ser adicionado à fila para o próximo $i$. Ele é usado para evitar adicionarmos um mesmo pixel duas vezes à fila.

- int iParada[] com tamanho $|\mathcal{D}|$. Este vetor só é usado se $\mathbb{B}$ for cíclico e indica para qual valor de $i$ o pixel $p$ não precisa mais ser dilatado.

- Lista unioes Armazena triplas da forma $(i, p, q)$, que indicam quais elementos $p$ e $q$ se uniram e sob qual passo $i$. É usado apenas se quisermos reconstruir os CCs para um $i$ qualquer. 

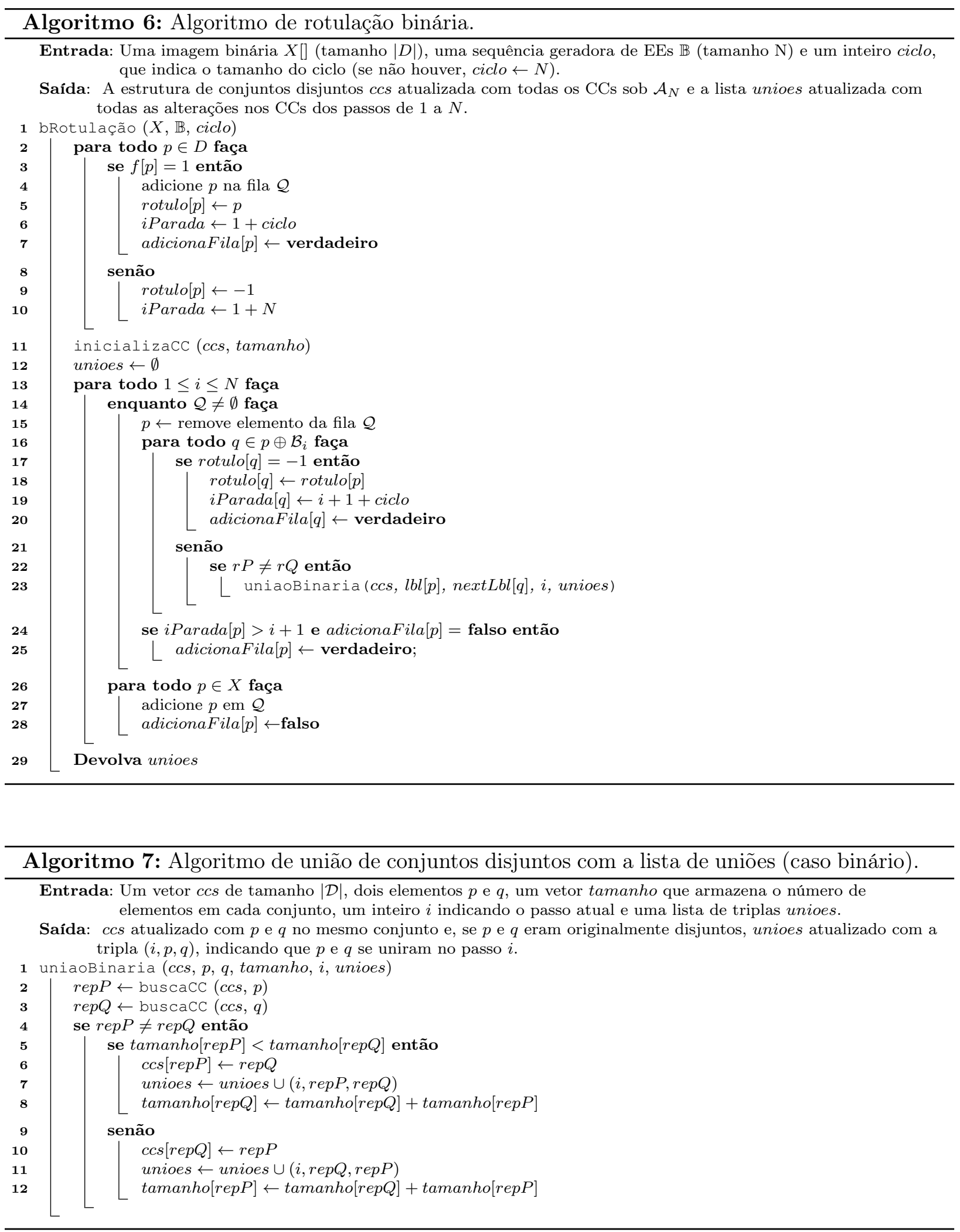

O Algoritmo 6 processa todos os pixels de objeto de $X$ para um dado $i$, a fim de computar os CCs de $X$ sob $\mathcal{A}_{i}$ antes de passar para $i+1$. A fila organiza o $p$ que está sendo processado e o laço a partir da linha 16 é responsável por propagar o rótulo armazenado em $p$ para os vizinhos de $p$ sob $\mathcal{B}_{i}$ (encontrando os novos vizinhos $q$ de $p$ sob $\mathcal{B}_{i}$ ). As linhas 17 e 21 verificam se $p \oplus \mathcal{B}_{i}^{\Sigma} \cap q \oplus \mathcal{B}_{i}^{\Sigma} \neq \emptyset$ e, se for o caso, sabemos que eles são vizinhos sob $\mathcal{A}_{i}$ e podemos unir os respectivos elementos na estrutura de conjuntos disjuntos CCS (linha 24). Como ao final de um passo $i$ sabemos que a fila está vazia, podemos usar a própria fila para computar o passo $i+1$, pois sabemos que os elementos 
que devem entrar na fila para o próximo passo estão dentro da estrutura adicionaFila. A função uniaoBinaria consiste do algoritmo uniaoCC modificado para adicionar a tripla $(i, p, q)$ à lista unioes sempre que $(p, q)$ se tornam vizinhos sob $\mathcal{A}_{i}$.

\subsection{Computação eficiente dos rótulos (caso geral)}

Toda a teoria vista até agora é válida para o caso binário e poderia ser aplicada em imagens em níveis de cinza ao decompormos uma imagem em conjuntos de níveis. Porém, como há uma relação de inclusão entre os conjuntos de níveis, muitas informações obtidas em alguns conjuntos podem ser usadas para obter informações nos outros níveis, o que permite armazenar tanto a árvore quanto os rótulos dos pixels de forma mais eficiente.

Como agora estamos tratando o caso em níveis de cinza, vamos usar a estrutura pais definida na Seção 4.1.1 para representar a árvore.

\subsubsection{União de CCs na representação compacta}

Sabemos que só podem ocorrer alterações na árvore se houverem alterações nos CCs. Porém, como a árvore engloba vários conjuntos de níveis, uma alteração em um CC de um conjunto de nível pode acarretar em alterações nos conjuntos de níveis anteriores. Então, nesta seção analisaremos como realizar estas uniões de forma a atualizar corretamente as relações de parentesco da árvore.

Assim, sejam $p$ e $q$ dois pixels desconexos sob $\mathcal{A}_{i}$ mas que se tornarão vizinhos sob $\mathcal{A}_{i+1}$. Suponha, sem perda de generalidade, que $f(p) \geq f(q)$. O primeiro fato que devemos observar é que, se $f(p)>f(q)$, então para todo conjunto de nível com índice maior que $f(q)$, $q$ se torna pixel de fundo e, portanto, não há união a ser feita.

Assim sendo, podemos começar o processo de união a partir do nível $f(q)$. Além disso, como estamos usando o passo $i$ para construir a árvore de $i+1$, é possível que, para algum nível de cinza $j(0 \leq j \leq f(q)), p$ e $q$ já estejam unidos em um mesmo CC e, neste caso, podemos parar de processar as uniões, pois todos os CCs que contém $p$ nos conjuntos de níveis com índice entre 0 e $j$ também contém $q$. Portanto, podemos restringir as uniões aos níveis de cinza entre $j$ e $f(q)$.

Suponha que temos os nós de $\mathcal{T}\left(f, \mathcal{A}_{i}\right)$ e queremos encontrar os nós de $\mathcal{T}\left(f, \mathcal{A}_{i+1}\right)$. Sejam $\mathcal{N}_{\mathcal{A}_{i}}(p)$ o nó de $\mathcal{T}\left(f, \mathcal{A}_{i}\right)$ que contém $p, \mathcal{N}_{\mathcal{A}_{i}}(q)$ o nó que contém $q$ e $\mathcal{N}_{\mathcal{A}_{i}}\left(p^{\prime}\right)$ o nó que é pai de $\mathcal{N}_{\mathcal{A}_{i}}(p)$. Pela Prop. 2.4.11, sabemos que, para todos os níveis de cinza entre $f(p)$ e $f\left(p^{\prime}\right)$, os CCs que estão implícitos na max-tree são todos iguais a $C C\left(\mathcal{N}_{\mathcal{A}_{i}}(p)\right)$.

Como vimos, não há união a ser feita nos níveis de cinza maiores que $f(q)$. Com relação ao nível de cinza $f\left(p^{\prime}\right)$, há somente duas possibilidades:

1. $f\left(p^{\prime}\right)>f(q)$ : neste caso, não haverá nenhuma união pois ainda estamos em um nível de cinza maior que $f(q)$.

2. $f\left(p^{\prime}\right) \leq f(q)$ : neste caso, há pelo menos uma união que ocorre no nível $f(q)$. Se $f\left(p^{\prime}\right)=f(q)$, então temos que unir os nós $\mathcal{N}_{\mathcal{A}_{i}}(q)$ e $\mathcal{N}_{\mathcal{A}_{i}}\left(p^{\prime}\right)$ em um único nó. Senão, no nível de cinza $f\left(p^{\prime}\right)$, $q$ é pixel de objeto e $C C\left(\mathcal{N}_{\mathcal{A}_{i}}(q)\right)$ será subconjunto de $C C\left(\mathcal{N}_{\mathcal{A}_{i+1}}\left(p^{\prime}\right)\right)$. Na nossa representação da max-tree, tal operação será feita fazendo com que $\mathcal{N}_{\mathcal{A}_{i+1}}(q)$ seja descendente de $\mathcal{N}_{\mathcal{A}_{i+1}}\left(p^{\prime}\right)$.

As duas possibilidades acima são suficientes para atualizar todas as relações de parentesco de nós que continham $p$ e $q$ em $\mathcal{T}\left(f, \mathcal{A}_{i}\right)$ de forma recursiva: basta repetirmos a operação até encontrarmos o primeiro nó que já continha $p$ e $q$ unido em um único CC (no pior caso, tal nó é o nó raiz), sempre fazendo valer a relação $f(p) \geq f(q)$. No primeiro caso, isto é feito atribuindo $p=p^{\prime}, p^{\prime}=$ pai de $p^{\prime}$ e mantendo $q$ intacto. No segundo caso, isto é feito atribuindo $p=q, p^{\prime}=$ pai de $q$ e $q=p^{\prime}$.

O algoritmo de união de CCs disjuntos é apresentado no Algoritmo 8. O algoritmo não apenas realiza as uniões quando necessário, mas também detecta quando os CCs já satisfazem um relação de inclusão entre eles e, neste segundo caso, a árvore não é alterada. Este algoritmo é semelhante ao algoritmo connect apresentado no trabalho de Wilkinson et.al. [ $\left.\mathrm{WGH}^{+} 08\right]$, que é usado para 
unir duas max-trees computadas concorrentemente em diferentes partes de uma imagem a fim de obter a max-tree que corresponde a união das partes.

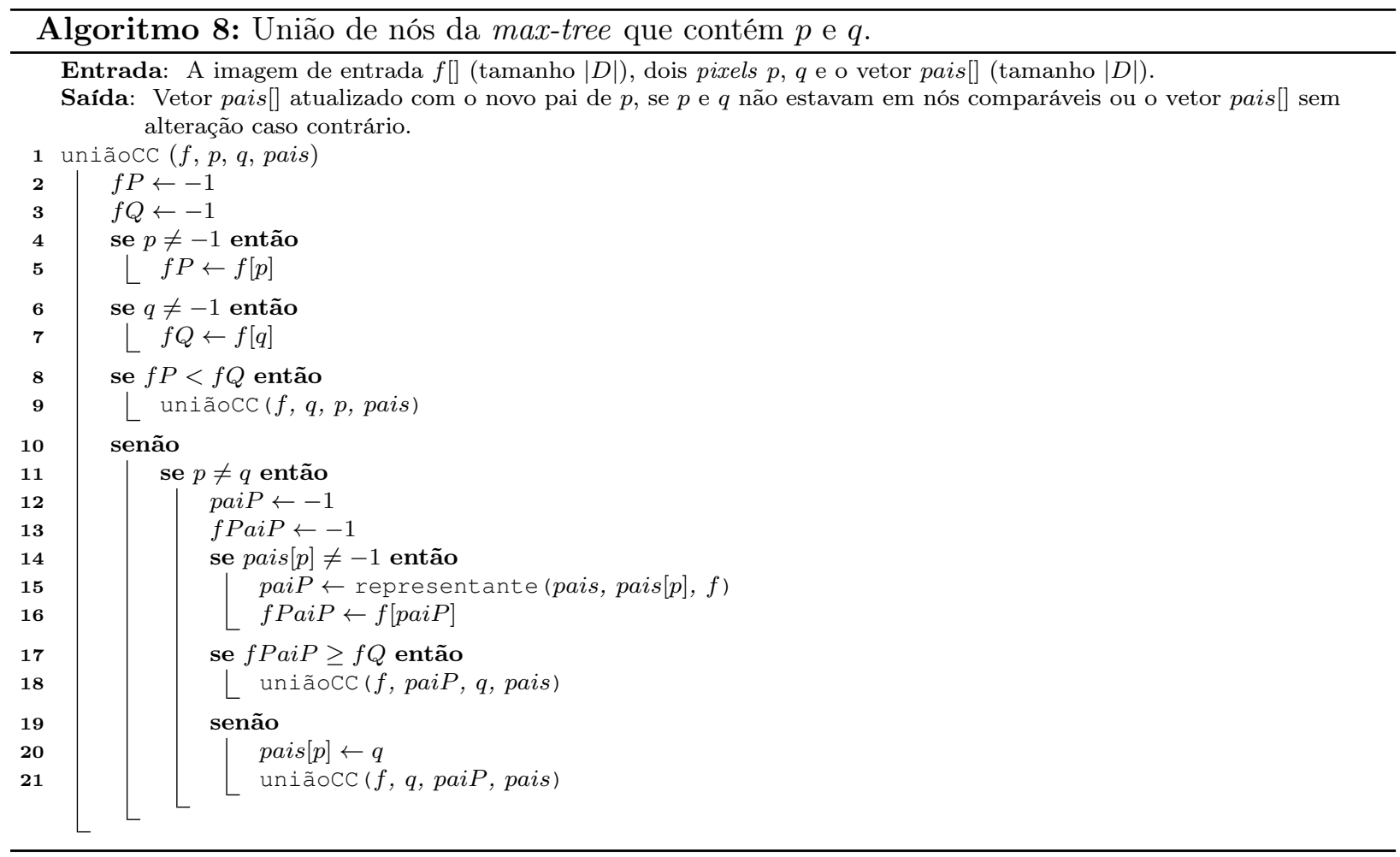


Exemplo 4.3.1. Este exemplo ilustra a simulação do Algoritmo 8 para a imagem em níveis de cinza $f$ unidimensional vista nos exemplos anteriores e repetida na Fig. 4.2. Abaixo da imagem é mostrada a estrutura pais atualizada para a vizinhança $\mathcal{A}_{1}$, que corresponde a uma vizinhança $3 \times 1$. $\dot{A}$ direita é exibida a max-tree de $f$ sob $\mathcal{A}_{1}$ (lembrando que a única informação que armazenamos é o vetor pais. Esta representação em forma de árvore só está ilustrada para fins didáticos). Os pixels na cor preta em cada nó indicam que aquele é o menor nó que os contém. Pixels na cor cinza indicam que eles foram herdados dos nós descendentes e não serão explicitamente armazenados sob uma representação compacta da árvore quando a construirmos.
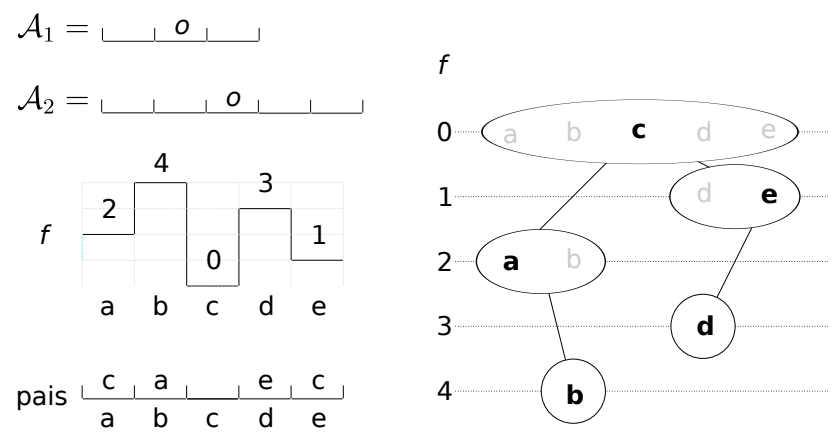

Figura 4.2: Max-tree da imagem $f$.

Tendo tal árvore, suponha que queremos atualizá-la para obtermos a max-tree de $f$ sob a vizinhança $\mathcal{A}_{2}$, ou seja, a vizinhança $5 \times 1$. Note que, sob $\mathcal{A}_{2}, b$ e d passam a ser vizinhos. Assim, é necessário alterar a árvore de forma a refletir tal fato.

Como $f(b)>f(d)$, fazemos as atribuições $p \leftarrow b$ e $q \leftarrow d$. O pai de b, a quem chamamos de paiP, é o nó representado por a.

Pelas propriedades vistas, sabemos que existem CCs redundantes que seriam unidos entre os niveis de cinza menores ou iguais a $f(d)$ e maiores que $f(a)$. No exemplo, o único nivel de cinza que pertence a tal intervalo é 3 . Assim, deve ocorrer uma união entre os CCs que contém $b$ e d no nivel 3.

Devido à representação compacta, o nó que contém b no nivel 3 não é representado. Assim, a união é feita alterando a relação de parentesco, fazendo com que d seja o novo pai de b, pois o CC que conterá b e d (no nível 3) é superconjunto de b (do nível 4). Tal operação é feita no algoritmo na linha 15 e mostrada na Fig. 4.3, na primeira ilustração da esquerda para a direita.

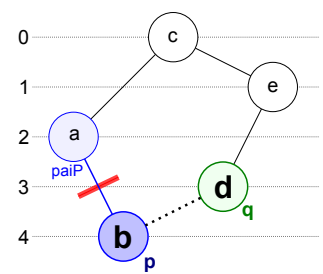

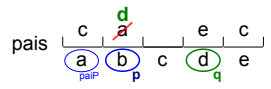

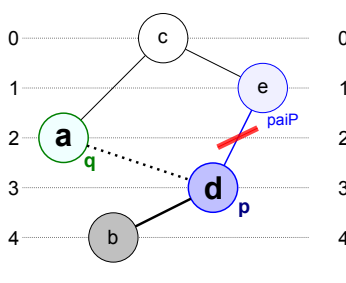

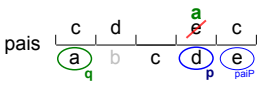

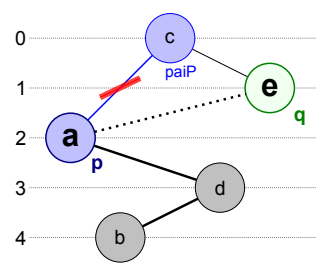

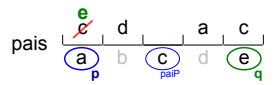

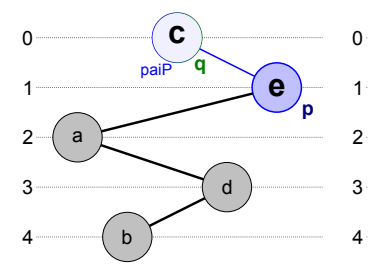

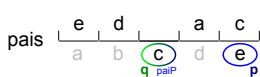

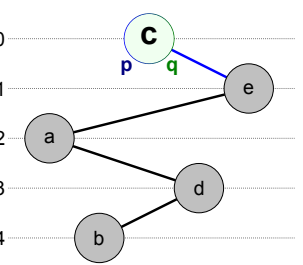

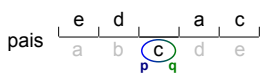

Figura 4.3: Simulação do algoritmo de união.

Com todas as uniões atualizadas para os niveis de cinza maiores ou iguais a 3, o algoritmo continua de forma recursiva atribuindo $p \leftarrow q, q \leftarrow$ paiP e paiP recebe o nó que é pai do $p$ atual, isto é, temos agora $p=d, q=a$ e paiP $=e$.

O mesmo processo se repete até que p e q sejam o mesmo nó (linha 11 do algoritmo). A simulação do algoritmo e os efeitos nas alterações das relações de parentesco podem ser vistas nas sequência de imagens da Fig. 4.3. Ao final da sequência de execuções, temos que $p=q=c$ e o algoritmo se encerra, tendo atualizado todos os CCs que contém os pixels $b$ e d.

O algoritmo de união deve ser processado para todo par de pixels que se tornaram vizinhos sob a nova vizinhança. No exemplo apresentado, não há mais alterações a ser feitas, de forma que a 
árvore obtida no final das uniões da Fig. 4.3 já representa a max-tree sob vizinhança $\mathcal{A}_{2}$. De fato, sabemos que não há mais nenhuma união a ser feita pois todos os nós da árvore são comparáveis entre si.

$O$ resultado do algoritmo comparando as árvores de $f$ sob as vizinhanças $\mathcal{A}_{1}$ e $\mathcal{A}_{2}$ podem ser vistas na Fig. 4.4. As arestas azuis mostram onde houve alteração nas relações de vizinhança $e$ estão indicadas também em azul na estrutura pais.
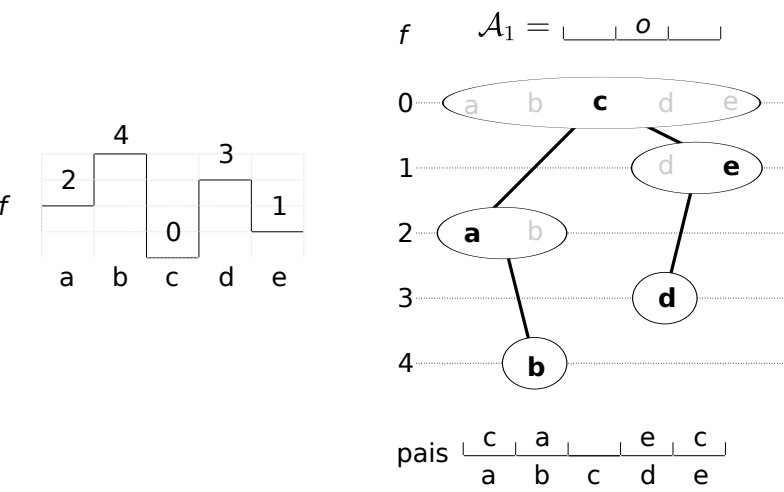

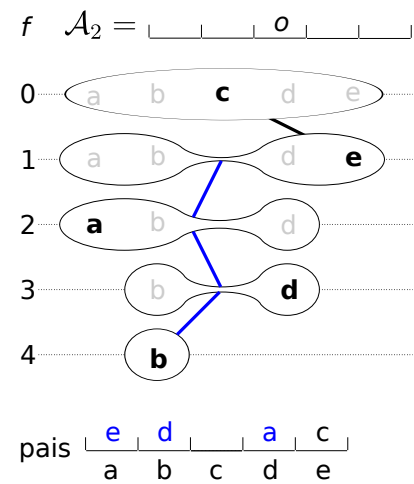

Figura 4.4: Max-tree da imagem $f$ para as vizinhanças $\mathcal{A}_{1}$ e $\mathcal{A}_{2}$.

\subsection{Algoritmo de rotulação}

\subsubsection{Visão geral}

A teoria vista na Seção 3.2 induz a um algoritmo de rotulação que permite encontrar os CCs de uma imagem em níveis de cinza $f$ para todas as vizinhanças de uma sequência gerada $\mathbb{A}$ :

1. Inicialmente, temos de gerar o caso base. Antes do algoritmo começar, não há nenhum vizinhança a ser considerada, então vamos assumir que cada pixel é um CC por si só.

2. Após inicializado, podemos fazer a atualização da função de rotulação para a vizinhança $\mathcal{A}_{1}$. Para tanto, atualizamos $\left[\operatorname{Rot}\left(f, \mathcal{B}_{1}^{\Sigma}\right)\right](p)$ para todo $p$ usando a Prop. 3.2.11. Processando todos os pixels do domínio, teremos a max-tree atualizada para $\mathcal{A}_{1}$, com a árvore representando os CCs de $f$ sob $\mathcal{A}_{1}$.

3. Com o passo $i=1$ pronto, basta repetir o mesmo processo para $\mathcal{A}_{2}$ e assim por diante, até a última vizinhança de $\mathbb{A}$.

O algoritmo acima pode ser desenvolvido a partir de propriedades vistas nos capítulos anteriores. Porém, assim como no caso binário, podemos aplicar algumas técnicas para melhorar o consumo de tempo e memória do algoritmo.

\subsubsection{Armazenamento eficiente da função de rotulação}

No caso binário, era possível reduzir o consumo de memória usando apenas um representante para cada $\left[\operatorname{Rot}\left(f, \mathcal{B}_{i}^{\Sigma}\right)\right](q)$. Tal representante era um elemento de cada conjunto disjunto, que era responsável por identificar a qual $\mathrm{CC}$ tal pixel $q$ pertence.

Como um único pixel pode pertencer a vários CCs para imagens em níveis de cinza, tal opção não é mais viável. Porém, dado um par de pixels $p_{1}$ e $p_{2}$, vimos na Seção 4.3 .1 um algoritmo que consegue unir os CCs em que $p_{1}$ e $p_{2}$ pertencem para todos os níveis de cinza relevantes. Em outras palavras, se soubermos o maior nível de cinza em que ocorre uma união entre os pixels $p_{1}$ e $p_{2}$, todas as outras uniões envolvendo este mesmo par de pixels podem ser inferidas através das relações de inclusão de CCs. Portanto, é suficiente armazenar, para cada $\left[\operatorname{Rot}\left(f, \mathcal{B}_{i}^{\Sigma}\right)\right](q)$, apenas um elemento 
dentro do conjunto $q \oplus\left(\mathcal{B}_{i}^{\Sigma}\right)^{t}$ de maior nível de cinza. Se a estrutura pais estiver sempre atualizada, podemos usá-la para detectar todos os outros nós a qual cada pixel pertence. Tal propriedade nos permite novamente utilizar apenas um elemento para cada representar a rotulação de cada pixel, reduzindo consideravelmente o consumo de memória.

Seja $r t$ esta estrutura que armazena apenas um rótulo por pixel. Podemos definir $r t$ para uma imagem em níveis de cinza como $\left[r t\left(f, \mathcal{B}_{i}^{\Sigma}\right)\right](q)=p$, onde $p$ é um elemento de $q \oplus\left(\mathcal{B}_{i}^{\Sigma}\right)^{t}$ de maior nível de cinza.

\subsubsection{Deteç̧ão de rótulos inalterados}

Assim como no caso binário, se soubermos que a sequência de elementos estruturantes possui alguma relação de inclusão de elementos estruturantes, podemos reduzir a complexidade do algoritmo evitando propagar rótulos de alguns pixels que sabemos que são desnecessários.

Nos restringiremos, tal como antes, ao caso em que a sequência gerada $\mathbb{B}$ é cíclica. No caso binário, cada pixel só precisava ser propagado $c$ vezes, onde $c$ é o tamanho do ciclo. Uma vez que um pixel propagava $c$ vezes, sabíamos que ele nunca mais poderia alterar os rótulos dos seus vizinhos.

Portanto, um rótulo só pode ser alterado quando um vizinho de nível de cinza maior é encontrado. Na prática, era exatamente a mesma coisa que ocorria no caso binário: quando um vizinho de nível maior que 0 era encontrado, o rótulo se alterava. Como existiam apenas dois níveis possíveis, após um ciclo completo, os rótulos não se alteravam mais simplesmente porque não havia nenhum pixel da imagem com nível maior que 1.

O que tal propriedade nos indica é o seguinte: no caso binário, um pixel que foi propagado por um ciclo completo nunca mais precisava ser propagado novamente. No caso em níveis de cinza, como existem vários níveis, um pixel que foi propagado por um ciclo completo não precisa ser mais propagado até que seu rótulo se altere novamente. Para todos os passos em que não há alteração de rótulos, podemos simplesmente ignorar os rótulos de tais pixels, reduzindo a complexidade geral do algoritmo.

\subsubsection{Processamento ordenado dos níveis de cinza}

Nos algoritmos usuais de construção das árvores, o processamento ordenado dos pixels de acordo com o nível de cinza, adicionando ligações à árvore apenas quando visitávamos um nó já processado, nos permitia manter a seguinte propriedade: qualquer relação de parentesco adicionada a max-tree nunca mais será removida posteriormente, sendo que novas alterações na árvore só podem adicionar novas arestas.

Embora esta propriedade só seja válida para a construção da max-tree com uma única vizinhança, ainda nos será útil manter esta propriedade pelo menos para o passo $i=1$, a fim de a construir esta primeira árvore da forma mais eficiente possível.

Para os passos seguintes, a execução em ordem de níveis de cinza ainda tem uma utilidade: a estrutura $r t$ que estamos usando armazena o elemento dentro da vizinhança de maior nível de cinza. Executando o algoritmo na ordem, garantimos que o primeiro pixel que visitar um vizinho $q$ com certeza será o elemento de maior nível de cinza e, neste instante, podemos atualizar $\left[r t\left(f, \mathcal{B}_{i+1}^{\Sigma}\right)\right](q)$ se assim for necessário, tendo a certeza de que ele não poderá mais ser alterado. 


\subsubsection{Algoritmo}

Baseado nas ideias vistas ao longa desta seção, podemos construir um algoritmo eficiente que constrói a max-tree de uma imagem em níveis de cinza para qualquer vizinhança dentro de $\mathbb{A}$. $\mathrm{O}$ algoritmo utilizará o seguinte conjunto de estrutura de dados:

- int $r t[]$ com tamanho $|\mathcal{D}|$. É a função $r t$ apresentada anteriormente.

- int rtProx [] com tamanho $|\mathcal{D}|$. Consiste da função $r t$ que será usada no passo $i+1$.

- int pais[] com tamanho $|\mathcal{D}|$. É uma estrutura que será usada para armazenar as relações de parentesco e de pixels no mesmo nó.

- Fila $\mathcal{Q}$ com prioridade maior dada a pixels de níveis de cinza mais altos. É usada para garantir a execução ordenada por níveis de cinza.

- boolean adicionaFila[] com tamanho $|\mathcal{D}|$. Este vetor indica se o pixel $p$ deve ser adicionado à fila para o próximo $i$. Ele é usado para evitar adicionarmos um mesmo pixel duas vezes à fila.

- int iParada[] com tamanho $|\mathcal{D}|$. Este vetor só é usado se $\mathbb{B}$ for cíclico e indica para qual valor de $i$ o pixel $p$ não precisa mais ser dilatado.

- Lista unioes. Armazena triplas da forma $(i, p, q)$, que indica quais elementos $p$ e $q$ se uniram e sob qual passo $i$. É usado apenas se quisermos reconstruir os CCs para um $i$ qualquer.

- int $f r t[]$ com tamanho $|\mathcal{D}|$. Inicialmente, consiste da imagem original $f$. frt se altera conforme a execução do algoritmo de forma a armazenar em $f r t[p]$ o valor de $f\left(\left[r t\left(f, \mathcal{B}_{i+1}^{\Sigma}\right)\right](p)\right)$. 


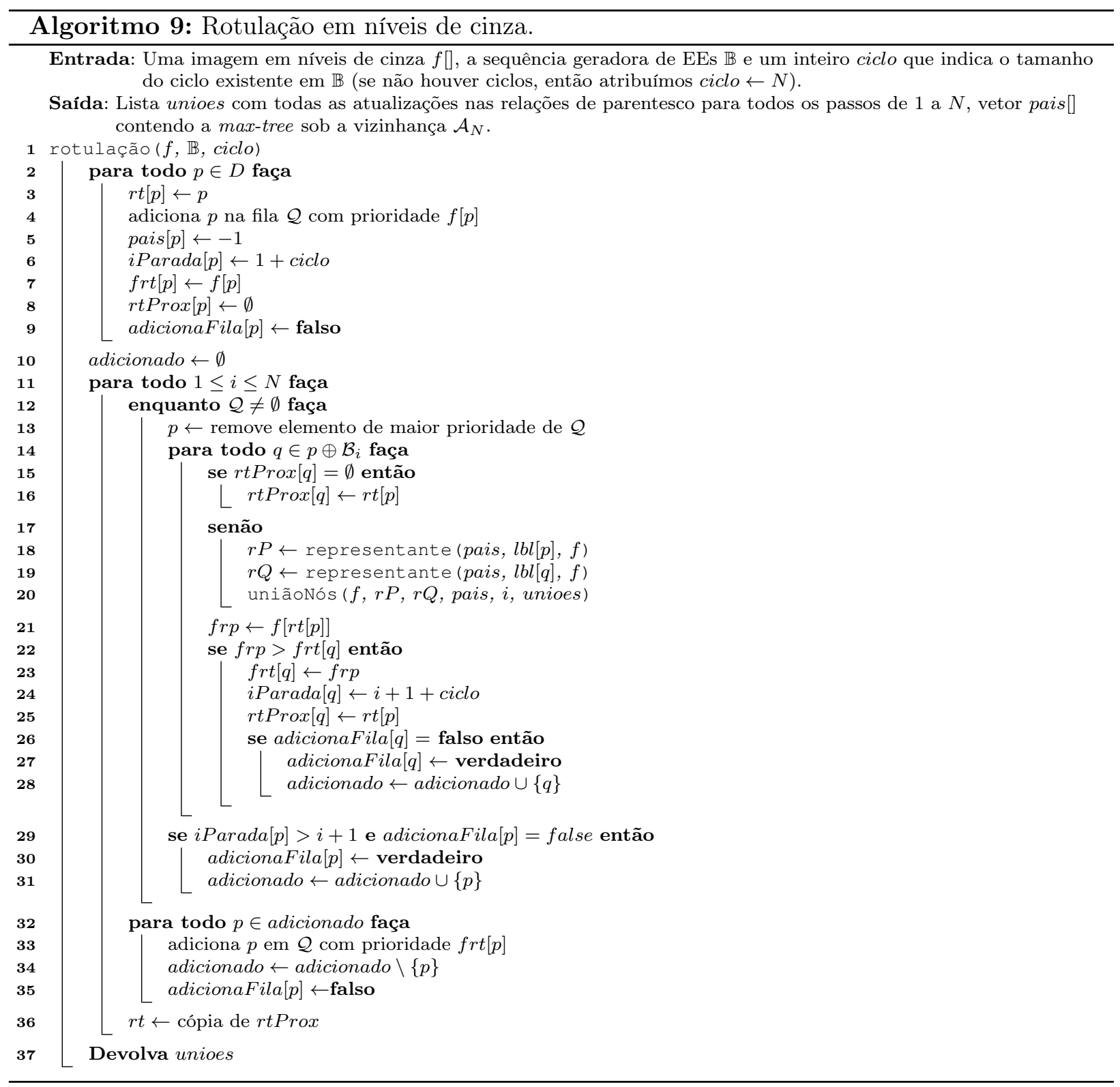

O Algoritmo 9 apresentado é bem parecido com o algoritmo para o caso binário. A grande diferença consiste na chamada da função uniãoNós, que será usada para juntar nós originalmente disjuntos em um mesmo nó. Por completude, esta função uni ãoNós é apresentada no Algoritmo 10, mas ele simplesmente consiste do algoritmo uniãoCC modificado para incluir as triplas indicando todas as alterações nas relações de parentesco.

O algoritmo funcionaria normalmente se inicializássemos rtProx como uma cópia de $r t$, mas atribuímos $\emptyset$ (linha 8) para todas as posições do vetor para garantir a ordem de execução para $i=1$. Tal medida faz com que um par de pixels vizinhos $(p, q)$ só seja considerado quando um dos elementos entre $p$ e $q$ já saiu da fila e o outro é o elemento sendo propagado no momento, garantindo que vale o invariante de que nenhuma aresta da max-tree se modifique depois de ser adicionada (no passo $i=1$ ). De fato, a linha 15 do algoritmo só é processada para $i=1$.

Como dito anteriormente, a função uni ãoNós é responsável também por verificar se é necessário unir nós ou não, de forma que podemos chamar a função sem nos preocuparmos em fazer tal verificação de antemão.

Por fim, a comparação da linha 26 consiste do caso em que há uma mudança de rotulação para um dado pixel. Neste caso, é necessário re-adicionar o elemento à fila, mesmo que ele já tenha terminado um ciclo, o que nunca acontecia no caso binário. A linha 29 garante que um elemento que não processou todo um ciclo sempre vai estar presente na fila. 


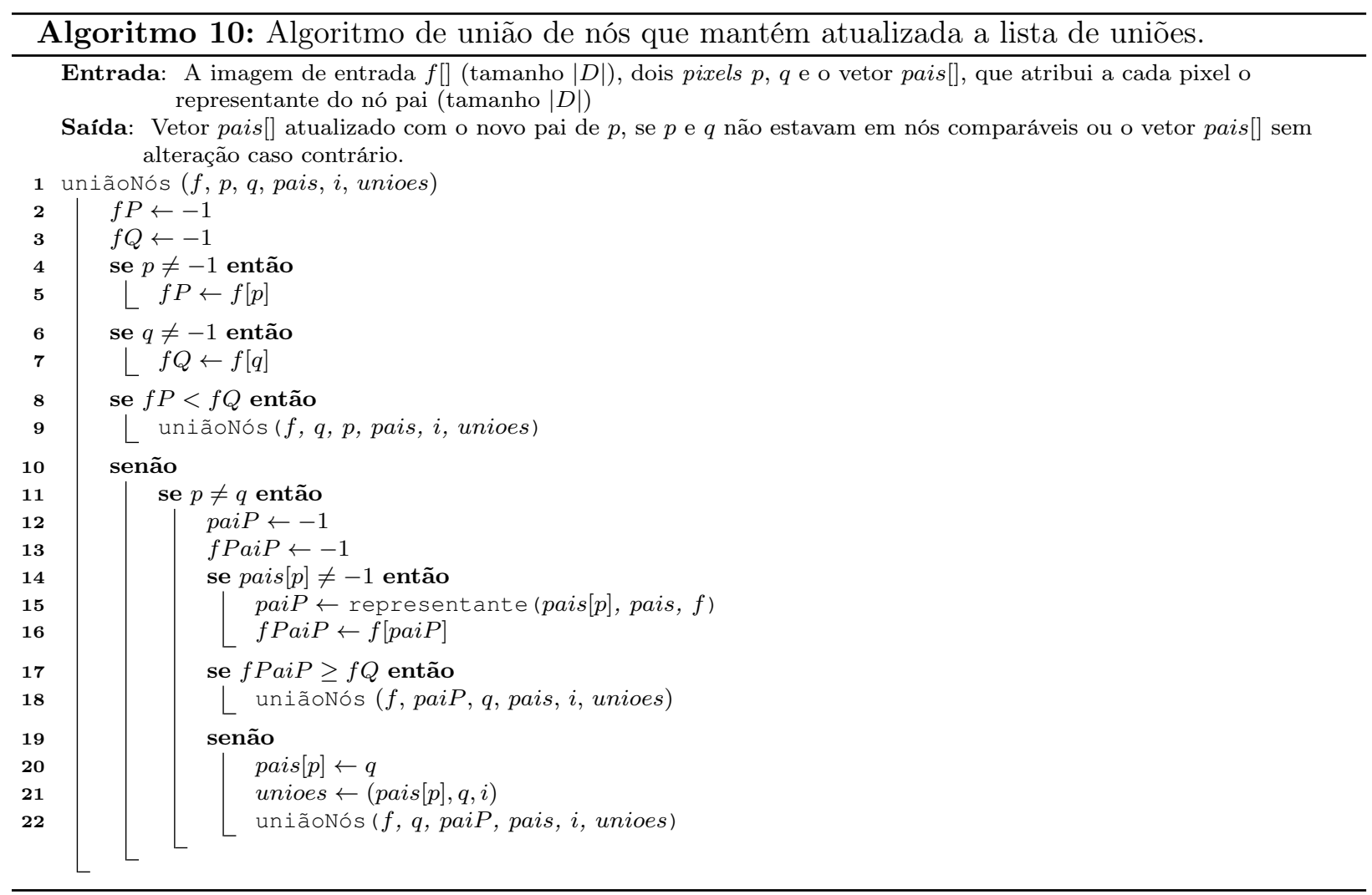

A fim de exemplificar o funcionamento do algoritmo, segue abaixo a simulação do algoritmo 9 para a imagem $f$ da Fig. 4.5:
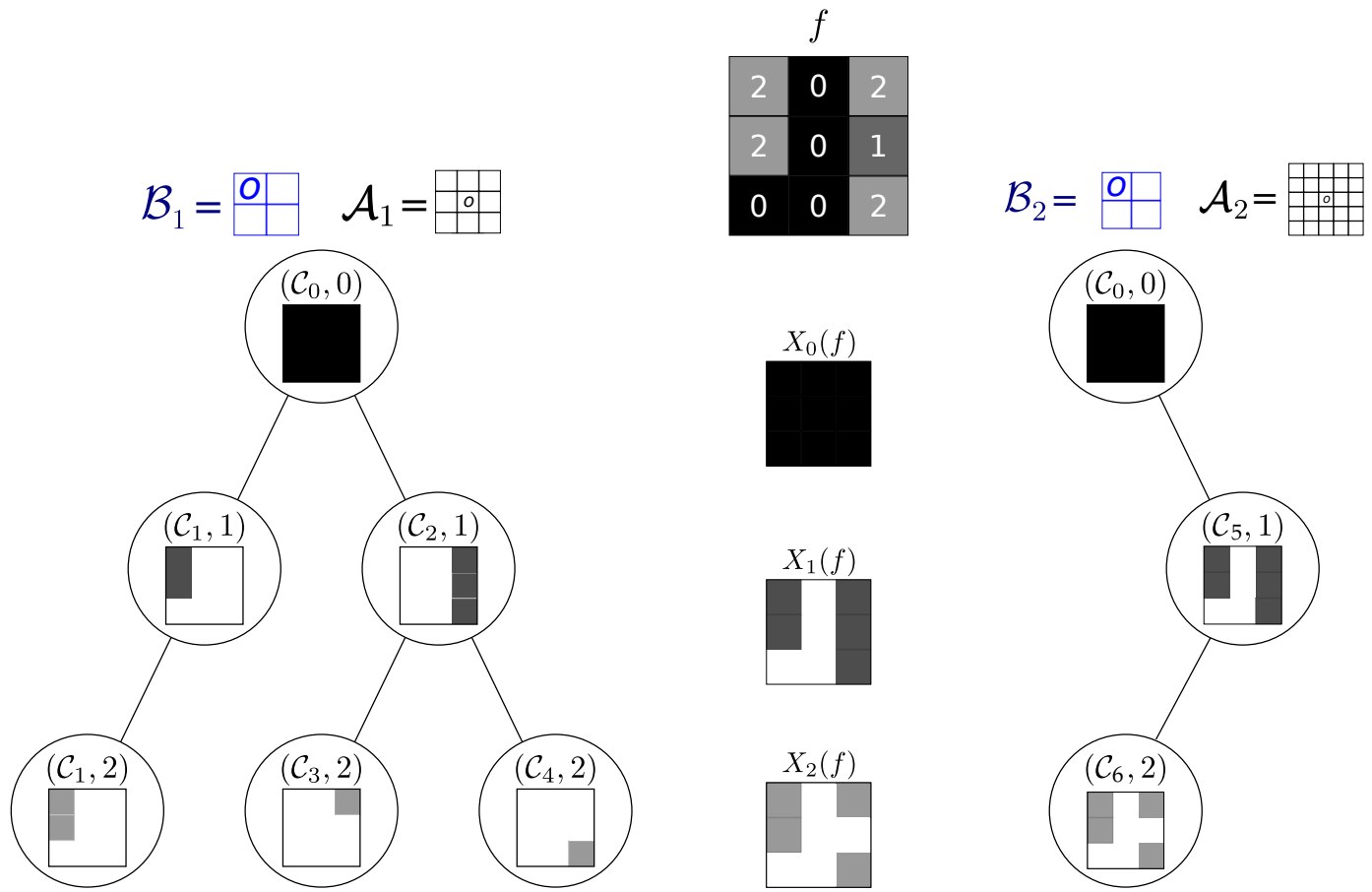

Figura 4.5: Árvores de componentes para a imagem $f$ e as vizinhanças $\mathcal{A}_{1}$ e $\mathcal{A}_{2}$ apresentadas. 
Exemplo 4.4.1. Neste exemplo, queremos construir, usando o algoritmo proposto, as max-trees que representam as árvores de componentes sob as vizinhanças $\mathcal{A}_{1}$ e $\mathcal{A}_{2}$ da Fig. 4.5. Para tanto, será necessário representar as estruturas de dados que representam as rotulações e a max-tree. A representação gráfica destas estruturas é apresentada na Fig. 4.6.

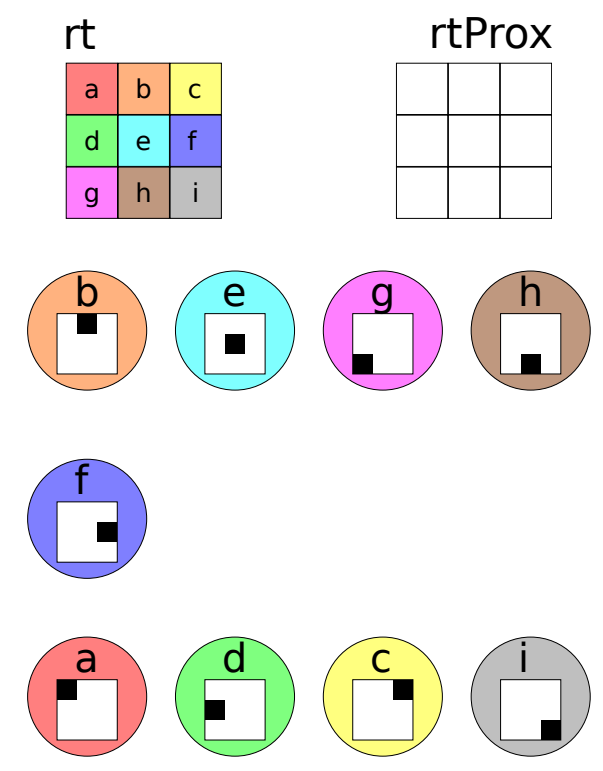

Figura 4.6: Estruturas de dados representando rt, rtProx e a max-tree.

Ao topo da Fig. 4.6 são mostradas as estruturas rt e rtProx, que indicarão, respecticvamente, o valor de $\operatorname{Rot}\left(f, \mathcal{B}_{i-1}^{\Sigma}\right)$ e $\operatorname{Rot}\left(f, \mathcal{B}_{i}^{\Sigma}\right)$ para cada pixel da imagem. Inicialmente consideraremos que cada pixel é um CC por si só, e rt[p] será inicializado como o próprio p. Queremos computar rtProx, e para o primeiro passo, a estrutura é inicializada vazia. Por fins didáticos, a cada pixel é atribuída uma cor diferente, a fim de se facilitar a visualização de intersecção entre vizinhanças de pixels.

Ainda na Fig. 4.6, é apresentada na parte inferior a árvore na forma de conjuntos disjuntos. Cada conjunto consiste de seu identificador e um apontador, que é inicializado vazio, indicando que cada pixel é, inicialmente, um CC por si só. Os quadrados dentro de cada conjunto disjunto indicam os pixels que pertencem a cada um deles. Note que as cores atribuídas a cada conjunto disjunto representam a cor do elemento canônico. Os conjuntos estão divididos em linhas, cada uma representando um nivel de cinza diferente. Assim sendo, apontadores na horizontal indicarão dois pixels pertencentes ao mesmo nó, enquanto que apontadores na vertical indicarão uma relação de inclusão.

A fim de simular o algoritmo, é necessário adicionar os pixels em uma fila de prioridades de acordo com os seus respectiveis niveis de cinza. Neste exemplo, consideraremos uma ordem da esquerda para direita e de cima para baixo (raster) para ordenar os pixels, de forma que a maior prioridade será dada ao pixel vermelho (a), seguida do pixel amarelo (c), verde (d) e assim por diante.

Assim sendo, suponha que extraímos o primeiro elemento da fila de maior prioridade (a). De acordo com a teoria e o algoritmo construído, é necessário propagar o rótulo de a para todos os vizinhos de a, $a \oplus \mathcal{B}_{1}$, a fim de indicar que todos eles são vizinhos de a sob $\mathcal{B}_{1}$. Isto é feito a partir da linha 14 do algoritmo 9 e, em particular, a propagação dos rótulos é feita na linha 16. Esta propagação do rótulo de a é ilustrada na Figura 4.7

$\mathrm{Na}$ Fig. 4.7 é mostrado o estado anterior de rtProx e o elemento de maior prioridade no momento (à esquerda), o conjunto de vizinhos de a sob $\mathcal{B}_{1}$ (no centro) e o resultado da rotulação (à direita), com a cor vermelha indicando todos os pixels que receberam o rótulo de a (isto é, para os pixels $q$ marcados em vermelho, temos agora rtProx $[q]=a$. 

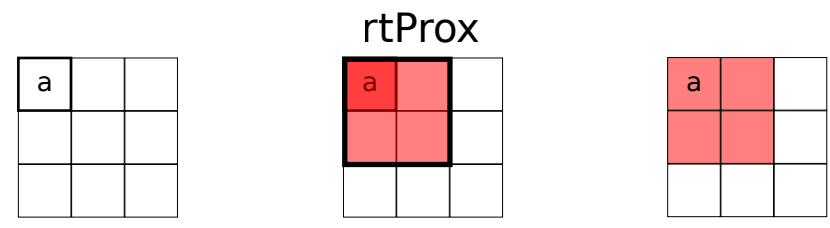

Figura 4.7: Propagação do rótulo de a para seus vizinhos.

Como ainda não houve nenhuma intersecção, o algoritmo continua para o próximo elemento na fila de maior prioridade (c) e o mesmo processo se repete. A propagação do rótulo de c é mostrada na Fig. 4.8.
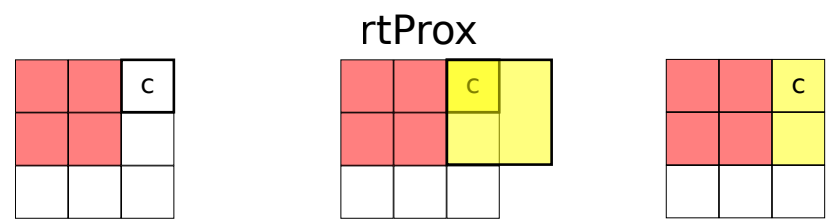

Figura 4.8: Propagação do rótulo de c para seus vizinhos.

Novamente, não há nenhuma intersecção e o algoritmo continua para o próximo elemento da fila (d). A propagação de d é mostrada na Fig. 4.9.
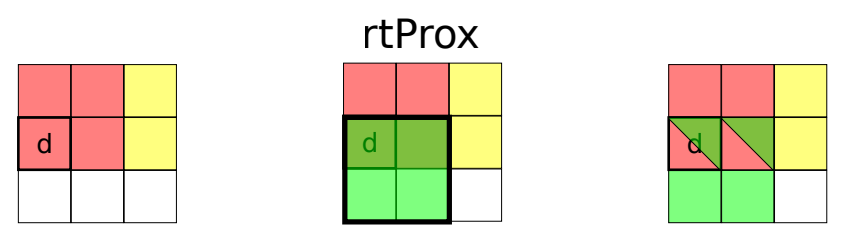

Figura 4.9: Propagação do rótulo de d para seus vizinhos.

Quando propagamos o rótulo de $d$, são encontrados vizinhos já rotulados. De acordo com a teoria, isso indica que os rótulos que se intersectaram são, na realidade, vizinhos sob $\mathcal{A}_{1}$ e podemos atualizar a árvore de acordo. No algoritmo, isto é feito nas linhas de 17 a 20, onde é chamada a função de união de nós da árvore. Neste caso em particular, sabemos que os pixels vermelho (a) e verde (d), são vizinhos sob $\mathcal{A}_{1}$ e serão unidos em um único conjunto disjunto. O resultado da operação de união é apresentado na figura 4.10. 


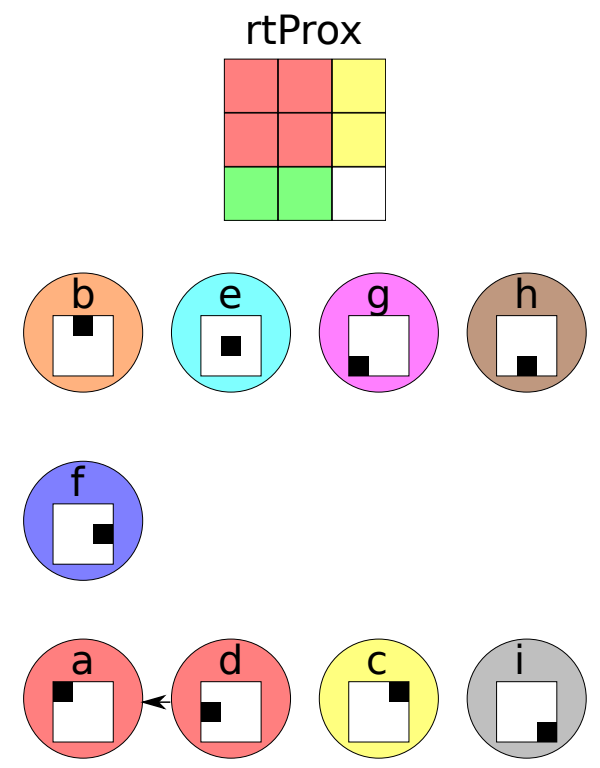

Figura 4.10: Resultado da propagação do rótulo de d.

O algoritmo de união fará com que d aponte para a na estrutura de conjuntos disjuntos, indicando que eles pertencem ao mesmo nó. Com esta informação atualizada na árvore, podemos manter como rótulo dos pixels em que ocorreram a intersecção apenas o elemento de maior nivel cinza, tal como explicado na Seção 4.4.2. Devido à execução ordenada em niveis de cinza, como justificado na Seção 4.4.3, não é necessário alterar o rótulo, pois o primeiro vizinho a propagar o rótulo sempre é o vizinho de maior nível. Portanto, para os pixels $d$ e e, onde ocorreram as intersecções, não há alteração nos rótulos (Fig. 4.10).

O algoritmo continua para os demais pixels na fila de prioridades. Sempre que dois rótulos diferentes se intersectam, a função de união é chamada e a árvore é atualizada. Ao final do processamento para $i=1$, teremos o resultado apresentado na Fig. 4.11. É fácil verificar que a árvore resultante, de fato, representa a árvore de componentes mostrada à esquerda da Fig. 4.5.
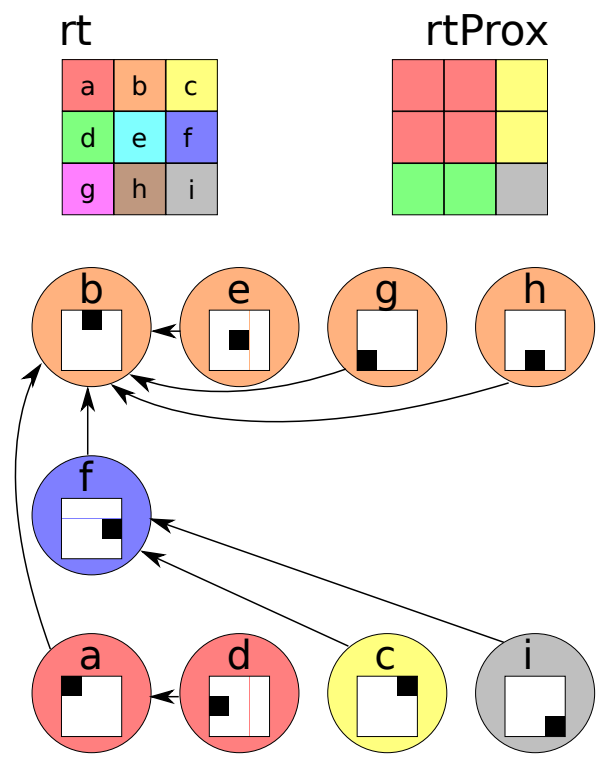

Figura 4.11: Estruturas de dados representando rt, rtProx e a max-tree ao final do passo $i=1$.

Quando a fila estiver vazia, todos os pixels que tiveram seu rótulo alterado (linhas 26 a 28) ou 
que não completaram um ciclo completo (linhas 29 a 31) serão readicionados à fila. No caso desta simulação, como o tamanho do ciclo é 1 (pois $\mathcal{B}_{1}=\mathcal{B}_{2}$ ), apenas os pixels $p$ que tiveram seus rótulos alterados são readicionados à fila, com prioridade frt $[p]$ (linhas 32 a 35). Por fim, trocamos as variáveis para termos rt representando $\operatorname{Rot}\left(f, \mathcal{B}_{1}^{\Sigma}\right)$.

Repetindo o processo para $i=2$, o pixel a não é adicionado à fila porque não teve seu rótulo alterado (devido ao que foi explicado na Seção 4.4.3: mesmo que propágassemos o rótulo de a para os vizinhos em $a \oplus \mathcal{B}_{2}$, os rótulos não se alterariam porque $a \oplus \mathcal{B}_{2}=a \oplus \mathcal{B}_{1}$ e os vizinhos de a já receberam o rótulo de a no passo anterior). Assim o elemento de maior prioridade na fila é $b$. A propagação do rótulo de b para os vizinhos é apresentada na Fig. 4.12.
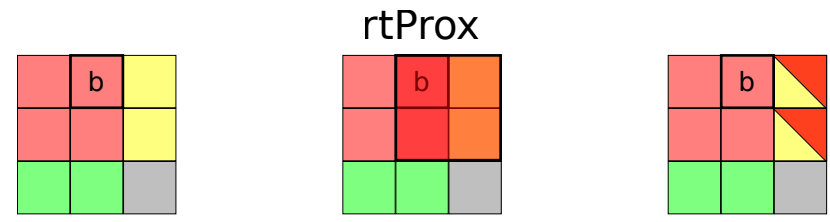

Figura 4.12: Propagação do rótulo de b para seus vizinhos no passo $i=2$.

Como se pode ver, há uma intersecção entre os pixels vermelho (a) e amarelo (c), indicando que a e c são vizinhos sob $\mathcal{A}_{2}$. A função de união é chamada para unir os nós que contém a e c em um único nó. Tal como antes, como a e c estarão no mesmo nó a partir de agora na árvore, o rótulo se mantém mesmo após a intersecção (temos que manter o rótulo de maior nível de cinza, mas tanto a quanto c possuem o mesmo nível). O mesmo fato ocorrerá quando e sair da fila: quando seu rótulo for propagado, haverá uma intersecção com o rótulo de $i$, e descobriremos que a e $i$ são vizinhos sob $\mathcal{A}_{2}$. Ao final do processo para $i=2$, teremos a max-tree apresentada na Fig. 4.13. Novamente, é simples observar que a max-tree apresentada representa a árvore de componentes desenhada à direita na Fig. 4.5.

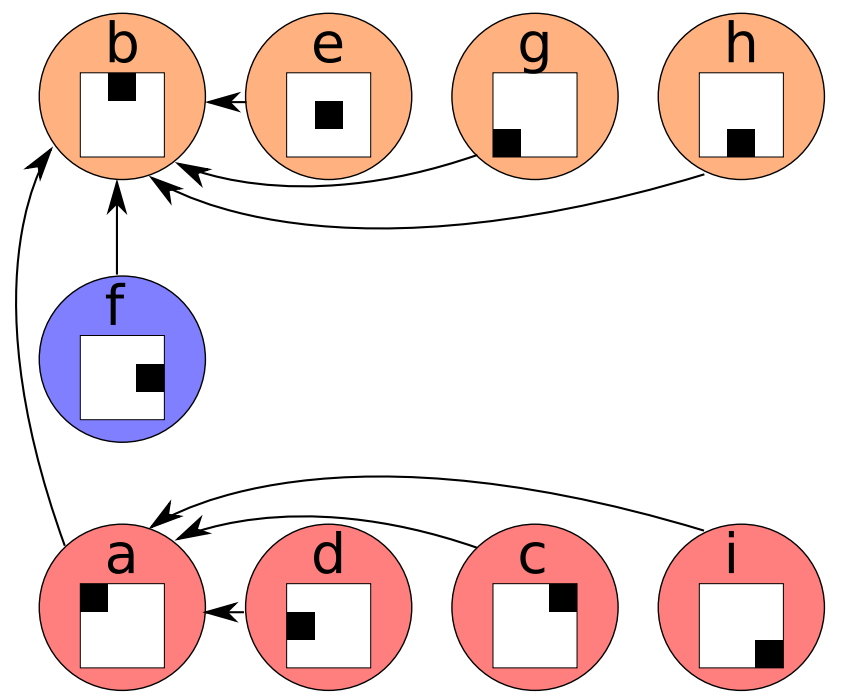

Figura 4.13: Max-tree para o passo $i=2$. 


\subsubsection{Reconstrução de max-trees intermediárias}

Ao final da construção da árvore final de vizinhança $\mathcal{A}_{N}$, também é armazenado na lista unioes o conjunto de todas as alterações nas relações de parentesco ao longo de todos os passos $i$ entre 1 e $N$. Fazendo uso desta lista, é possível reconstruir qualquer max-tree intermediária sob uma vizinhança qualquer $\mathcal{A}_{i}$. Para tanto, é suficiente fazermos uso de um vetor paiI que é inicializado vazio e é alterado de acordo com a lista unioes. Como sabemos que a lista está ordenada de acordo com o valor $i$ e os elementos dela são triplas da forma $(i, p, q)$ (onde cada tripla indica qual o novo pai de $p$ (no caso, $q$ ) no passo $i$ ), basta fazer a atribuição paiI $[p]=q$ para todas triplas de 1 até o índice $i$ que queremos reconstruir.

Ao final da construção da árvore, porém, como a função representante não é chamada, não é efetuada nenhuma compressão de caminhos e o custo de encontrar o representante de um nó pode ser demasiadamente caro porque o caminho que liga o pixel ao representante no vetor é muito longo.

\subsubsection{Canonização}

Ao final do processo de construção do algoritmo ou da criação de um vetor que exibe uma árvore de um passo intermediário restrito à uma vizinhança $\mathcal{A}_{i}$, é possível que o vetor pais não esteja canonizado, isto é, não satisfaça as propriedades vistas na Definição 4.1.1. Ao canonizarmos o vetor, garantimos que o custo da chamada da função representante é sempre constante.

Para que o vetor esteja canonizado, é necessário fazer com que todos as posições do vetor apontem para elementos canônicos. Isso pode ser feito de forma eficiente através do seguinte algoritmo:

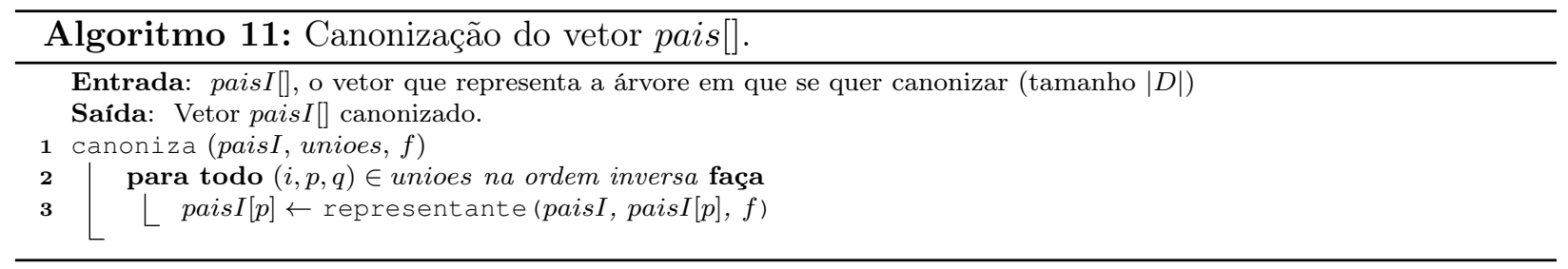

O algoritmo é semelhante ao apresentado por Berger et al. [BGL $\left.{ }^{+} 07\right]$. O processamento da lista em ordem inversa é importante porque o custo da função representante para os últimos elementos adicionados à lista é menor, pois eles têm maior chance de já estarem apontando para um elemento canônico. Com o vetor canonizado, é possível encontrar rapidamente a qual nó cada pixel pertence e é possível fazer computações na árvore.

Exemplo 4.4.2. A Figura 4.14 mostra um exemplo do efeito de se canonizar o vetor pais que contém as relações de parentesco: na figura, é dada uma imagem em níveis de cinza de largura 3 e altura 2, sendo que a árvore contém 3 níveis de cinza distintos. A vizinhança considerada no exemplo é a vizinhança $3 x 3$.

À esquerda, abaixo da figura, é dado um possivel resultado da reconstrução da árvore sob vizinhança 3x3, sem a canonização. As representações na forma de grafo são uma visualização do vetor pais apresentado imediadamente abaixo delas. Os pixels em negrito denotam os elementos canônicos de cada nó, enquanto que os vértices menores indicam os outros elementos. A disposição de vértices na vertical indica as relações de parentescos e vértices dispostos na horizontal indicam que eles pertencem a um mesmo nó, representado pelo elemento canônico.

É possivel notar que os pixels $s$ e u apontam para elementos que não são canônicos. Em particular, o custo de encontrar o elemento canônico do nó que contém u é mais alto por ser necessário percorrer todos os outros elementos do nó antes de encontrarmos o elemento canônico. Este custo é reduzido quando o vetor é canonizado, como podemos ver ao lado direito da Figura 4.14: o custo para encontrar o elemento canônico de cada pixel do dominio da imagem passa a ser constante a partir de então. Como a canonização é feita na ordem inversa da atualização das relações de parentesco, é possivel garantir que esta etapa precisa de no máximo duas chamadas recursivas para encontrar o elemento canônico para cada pixel: uma chamada é usada para encontrar o nó pai (que 
já foi canonizado anteriormente pela ordem das canonizações) e outro para encontrar o elemento do nó pai, que certamente é um elemento canônico.

\begin{tabular}{c|c|c}
\multicolumn{3}{l|}{ Imagem } \\
\hline $\mathrm{p}$ & $\mathrm{q}$ & $\mathrm{r}$ \\
$\mathrm{s}$ & $\mathrm{t}$ & $\mathrm{u}$
\end{tabular}

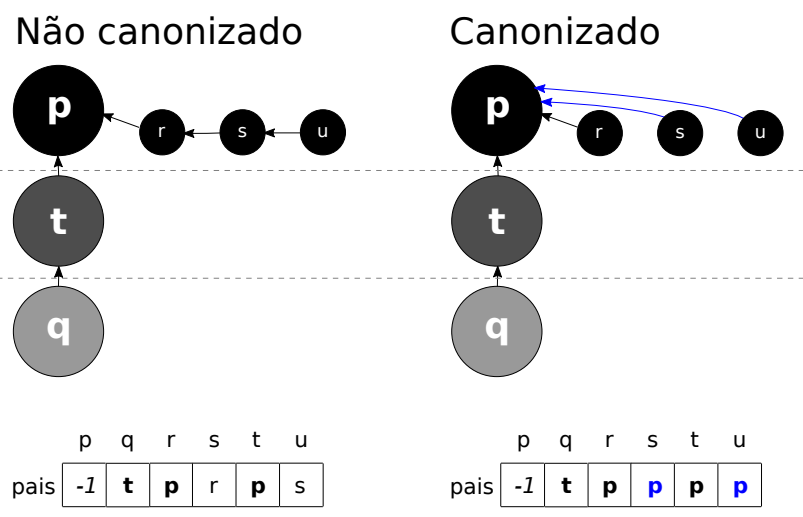

Figura 4.14: Exemplo do efeito do algoritmo de canonização em uma imagem em niveis de cinza.

\subsection{Cálculo de atributos na max-tree}

\subsubsection{Inversão das ligações da max-tree}

Com o algoritmo visto na Seção 4.4.5, temos a estrutura pais completamente atualizada para a vizinhança $\mathcal{A}_{i}$ dada. Embora tal estrutura já represente a árvore, os nós apontam em sentido aos nós pai. Para podermos fazer buscas na árvore, é importante que tenhamos também as ligações na ordem inversa.

Dessa forma, iremos reconstruir a max-tree na forma de um grafo, alocando estruturas para representar cada um dos nós existentes na estrutura pais e ligando os nós em ambas as direções quando há uma relação de parentesco entre um par de nós. Desta forma, com cada nó representado de forma explícita, é possível armazenar os atributos dentro do respectivo nó.

Assim sendo, um nó será um estrutura de dados com as seguintes informações:

- int pai: apontador para o nó pai;

- Lista filhos: lista com apontadores para cada um dos nós filhos;

- int nivelDeCinza: o nível de cinza do CC que o nó representa;

- Lista pixels: o conjunto de pixels cujo menor CC em que estão contidos é o CC que o nó representa;

- int representante: o representante do nó na estrutura pais;

- int atributos[]: vetor com valores de atributos;

- Lista CCsOriginais: lista com apontadores para os nós de árvores anteriores contidos neste nó (Seção 4.5.2). 
A partir do vetor pais, é possível criar esta estrutura tendo os apontadores para os pais e os filhos atualizados, além de ser podermos detectar o representante e os pixels que pertencem ao nó analisando quem representa cada pixel. O nível de cinza pode ser facilmente obtido uma vez que a lista de pixels esteja atualizada: basta analisarmos o nível de cinza dos pixels na imagem original.

Além de cada nó, alocaremos a estrutura mapaNos, que mapeará cada pixel da imagem no respectivo nó que está contido na árvore.

Após a alocação e a atualização dessas informações, os atributos de cada nó podem ser calculados através de uma busca em profundidade. Dependendo do atributo, é possível usar informações dos nós filhos para atualizar os valores de atributo. Em outros casos, devem-se reconstruir toda a CC para se poder calcular o atributo, o que é bem mais custoso computacionalmente.

\subsubsection{Análise de atributos de nós de outras árvores}

Uma vantagem de utilizarmos max-trees com diferentes vizinhanças é que podemos inferir relações de inclusão entre nós destas árvores. Além disso, sabemos que o nó de uma árvore com vizinhança maior é uma fusão de nós disjuntos da árvore gerada a partir da vizinhança menor. Se tivermos os atributos dos nós calculados, podemos calcular atributos com relação a variação destes atributos e obter um novo valor que nos diz relaciona de alguma forma os nós que se fundiram.

O exemplo abaixo mostra para um caso simples o cálculo de variância de um atributo:

Exemplo 4.5.1. Para este exemplo, usaremos a imagem $f$ e as vizinhanças mostradas na Fig. 4.15. As max-trees de $f$ para cada vizinhança também são explicitadas na mesma figura.

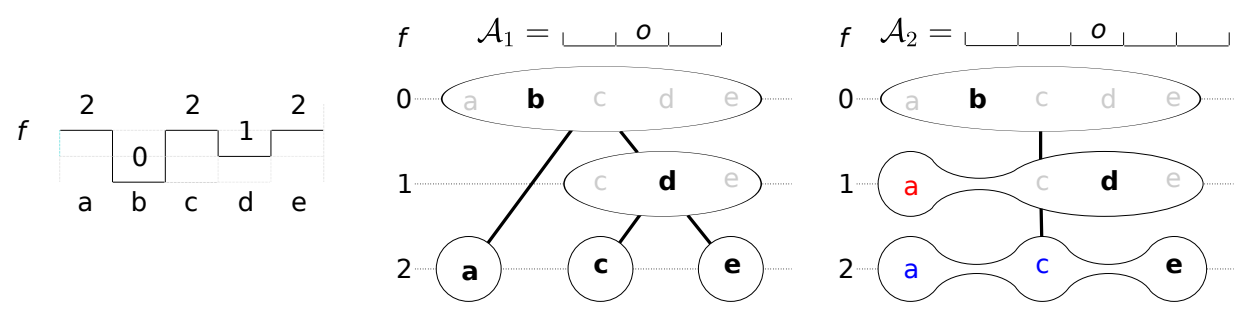

Figura 4.15: Imagem $f$ e a max-tree da imagem para as vizinhanças $\mathcal{A}_{1}$ e $\mathcal{A}_{2}$.

Suponha que queremos saber se nós que se fundiram na mudança da vizinhança de $\mathcal{A}_{1}$ para $\mathcal{A}_{2}$ têm área parecida. Seja $\mathcal{T}_{1}$ a max-tree gerada a partir da vizinhança $\mathcal{A}_{1}$ e $\mathcal{T}_{2}$ a max-tree gerada a partir de $\mathcal{A}_{2}$. Assim, vamos calcular a variância da área para os nós de $\mathcal{T}_{2}$ com relação aos nós de $\mathcal{T}_{1}$.

No exemplo, para a vizinhança $\mathcal{A}_{1}$, temos 5 nós, os quais chamaremos de $\mathcal{N}_{1}(a), \mathcal{N}_{1}(b), \mathcal{N}_{1}(c)$, $\mathcal{N}_{1}(d)$ e $\mathcal{N}_{1}(e)$, onde $\mathcal{N}_{1}(a)$ é nó que representa o menor $C C$ que contém a, isto é, vale que a $\in$ pixels $\left(\mathcal{N}_{1}(a)\right)$.

Assim sendo, os nós representam os seguintes CCs sob $\mathcal{A}_{1}$ :

- $C C\left(\mathcal{N}_{1}(a)\right)=\{a\} . \operatorname{Area}\left(\mathcal{N}_{1}(a)\right)=1$;

- $C C\left(\mathcal{N}_{1}(b)\right)=\{a, b, c, d, e\}$. Área $\left(\mathcal{N}_{1}(b)\right)=5$;

- $C C\left(\mathcal{N}_{1}(c)\right)=\{c\}$. Área $\left(\mathcal{N}_{1}(c)\right)=1$;

- $C C\left(\mathcal{N}_{1}(d)\right)=\{c, d, e\}$. Área $\left(\mathcal{N}_{1}(d)\right)=3$;

- $C C\left(\mathcal{N}_{1}(e)\right)=\{e\} . \operatorname{Area}\left(\mathcal{N}_{1}(e)\right)=1$;

Sob $\mathcal{A}_{2}$, temos os seguintes nós:

- $C C\left(\mathcal{N}_{2}(b)\right)=\{a, b, c, d, e\}$;

- $C C\left(\mathcal{N}_{2}(d)\right)=\{a, c, d, e\}$; 
- $C C\left(\mathcal{N}_{2}(e)\right)=\{a, c, e\}$;

Como $C C\left(\mathcal{N}_{2}(a)\right)=C C\left(\mathcal{N}_{2}(c)\right)=C C\left(\mathcal{N}_{2}(e)\right)$, precisamos de apenas um nó para representar o componente. Neste exemplo, estamos tomando o pixel e como representante do nó.

De acordo com a teoria, os CCs sob $\mathcal{A}_{2}$ são uniões de CCs sob $\mathcal{A}_{1}$. Assim, também podemos descrever os CCs sob $\mathcal{A}_{2}$ da seguinte maneira:

- $C C\left(\mathcal{N}_{2}(b)\right)=C C\left(\mathcal{N}_{1}(b)\right)$;

- $C C\left(\mathcal{N}_{2}(d)\right)=C C\left(\mathcal{N}_{1}(a)\right) \cup C C\left(\mathcal{N}_{1}(d)\right)$;

- $C C\left(\mathcal{N}_{2}(e)\right)=C C\left(\mathcal{N}_{1}(a)\right) \cup C C\left(\mathcal{N}_{1}(c)\right) \cup C C\left(\mathcal{N}_{1}(e)\right)$;

Calcular a variância da área com relação aos nós que de $\mathcal{T}_{1}$ que reconstroem $C C_{2}\left(\mathcal{N}_{1}(e)\right)$ corresponde a calcular a variância da área dos nós $\mathcal{N}_{1}(a), \mathcal{N}_{1}(c)$ e $\mathcal{N}_{1}(e)$. Neste caso, como todos os nós têm o mesmo valor, a variância é 0 .

Por sua vez, o cálculo da variância da área dos nós de $\mathcal{T}_{1}$ que recriam $C C\left(\mathcal{N}_{2}(d)\right)$ consiste em calcular a variância da área dos nós $\mathcal{N}_{1}(a)$ e $\mathcal{N}_{1}(c)$, o que resulta em 1.

Este valor que é calculado a partir dos atributos de nós de uma árvore de um passo anterior poderia ser facilmente obtido se não estivéssemos usando a representação compacta. Quando há uma união de nós originalmente disjuntos, tal união pode ser representada de duas formas: fundindo dois nós em um único (quando o nível de cinza dos nós é igual) ou alterando a relação de parentesco (conforme visto no exemplo da Seção 4.3.1).

O primeiro caso é simples. Na hora de calcular a variância, basta observarmos quais foram os nós que se fundiram para recriarmos o novo nó. Os nós que se fundiram devem ser adicionados à estrutura CCsOriginais do nó que os contém.

No segundo caso, é necessário combinar as informações das duas árvores para diferenciar quais nós são descendentes por causa da mudança de vizinhança e quais já eram descendentes mesmo na vizinhança anterior. Tal informação é importante porque, se um nó já era descendente na vizinhança anterior, o nó apenas estava incluso dentro de um nó maior e não havia nenhuma disjunção com relação ao nó ancestral.

Esta observação está diretamente relacionada com o algoritmo de atualização das relações de parentesco: se dois nós já eram comparáveis, então não havia necessidade de alterar nenhuma relação de parentesco. No caso atual, se os nós já eram comparáveis, então eles não eram disjuntos e há apenas o atributo do maior nó a ser levado em conta. Da mesma forma, quando os nós não eram comparáveis, era necessário alterar as relações de parentesco até atingirmos o pai em comum. Agora, se dois nós, digamos $\mathcal{N}_{1}$ e $\mathcal{N}_{2}$ não eram comparáveis, para todos os nós do caminho de $\mathcal{N}_{2}$ ao nó pai de $\mathcal{N}_{1}$, sabemos que todos eles são disjuntos com relação a $\mathcal{N}_{1}\left(\right.$ sob $\mathcal{A}_{1}$ ) e precisamos obter o valor dos atributos de todos estes nós.

Exemplo 4.5.2. É possivel observar este acontecimento na imagem do exemplo anterior: os pixels a, c e e possuíam todos o mesmo nivel. Sob $\mathcal{A}_{2}$, eles todos se tornavam conexos entre si e a variância podia ser facilmente calculada se armazenarmos no nó $\mathcal{N}_{2}(e)$ apontadores para os nós $\mathcal{N}_{1}(a), \mathcal{N}_{1}(c)$ e $\mathcal{N}_{1}(e)$.

Por outro lado, a área de $\mathcal{N}_{1}($ a) também precisa ser levada em conta no cálculo da variância da área para o nó $\mathcal{N}_{2}(d)$, mas as áreas de $\mathcal{N}_{1}(c)$ e $\mathcal{N}_{1}(e)$ não, apesar de todos os pixels destes nós estarem presentes em nós descendentes de $\mathcal{N}_{2}(d)$. Isso ocorre porque e e c já eram conexos com $d$ sob vizinhança $\mathcal{A}_{1}$, ao contrário do que ocorria com o pixel a. De fato, se observarmos quais nós eram disjuntos com relação a $\mathcal{N}_{1}(a)$ em $\mathcal{T}_{1}$, veremos que são todos os nós do caminhos de $\mathcal{N}_{1}(c)$ à raiz e de $\mathcal{N}_{1}(e)$ à raiz, que é o nó pai de $\mathcal{N}_{1}($ a). Neste caso, armazenamos na lista CCsOriginais do nó $\mathcal{N}_{2}(d)$ apontadores para os nós $\mathcal{N}_{1}(a)$ e $\mathcal{N}_{1}(d)$. 


\subsubsection{Atualização da lista CCsOriginais dos nós}

Tal como explicado anteriormente, a lista CCsOriginais de cada nó poderia ser atualizada durante as chamadas à função uniãoNós, pois precisamos detectar a mesma informação: quais CCs disjuntos passaram agora a serem conexos. Porém, naquele momento, ainda não possuíamos a estrutura da árvore com cada nó alocado. Além disso, atualizar CCsOriginais através de um pós-processamento tem a vantagem de podermos relacionar nós de quaisquer duas árvores entre si, não necessariamente consecutivas.

Assim, considere que são dadas duas árvores $\mathcal{T}\left(\mathcal{A}_{i}\right)$ e $\mathcal{T}\left(\mathcal{A}_{j}\right)$ com $\mathcal{A}_{i} \subset \mathcal{A}_{j}$ (ou seja, $i<j$ ). Chamaremos a árvore gerada a partir de $\mathcal{A}_{i}$ da árvore "menor", enquanto que a árvore de $\mathcal{A}_{j}$ será denominada da árvore "maior". Assim, o que queremos é encontrar os nós da árvore menor que, fundidos, geram os nós da árvore maior, para todos os nós da maior árvore.

O algoritmo que atualiza CCsOriginais com os apontadores para os nós da menor árvore é descrito abaixo.

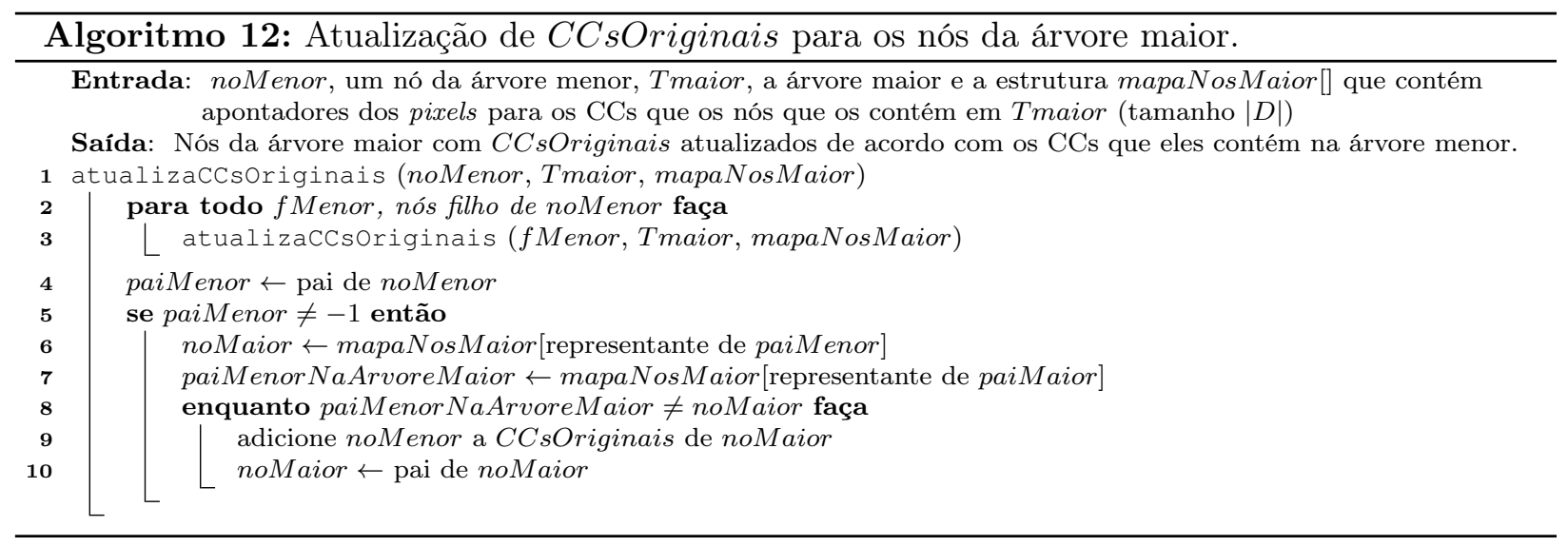

O algoritmo acima atualiza CCsOriginais para todos os nós da subárvore enraizada no nó que for usado para chamar a função. Para atualizar toda a árvore maior, basta chamar a função atualizaCCsOriginais passando o nó raiz da árvore menor como primeiro parâmetro.

Tendo CCsOriginais atualizado para todos os nós, basta usar os apontadores para obter os nós que estão contidos nele e seus atributos. Com base nisso, podemos calcular atributos em cima dos atributos dos nós de árvores anteriores, tal como foi feito no exemplo usando variância. 


\section{Capítulo 5}

\section{Resultados Experimentais}

Ao longo da última seção, foram apresentados o algoritmo desenvolvido para a construção da sequência de max-trees, além de outros algoritmos auxiliares, tais como o de cálculo de atributos dos nós das árvores. Neste capítulo, faremos uma análise dos algoritmos apresentados, mostrando ideias de aplicações onde ele pode ser utilizado e verificando o consumo de tempo esperado. Para embasar os resultados obtidos, uma análise teórica com relação à complexidade computacional do algoritmo é apresentada, mostrando que os tempos obtidos na prática estão de acordo com os resultados teóricos desenvolvidos.

\subsection{Comparação da variação de atributos}

Em muitos algoritmos de localização de textos em imagens, é importante comparar características de regiões candidatas próximas. Estas comparações parte do pressuposto que letras dentro de uma mesma palavra ou frase compartilham características em comum, que auxiliarão a diferenciar regiões textuais de ruídos.

Assumindo que caracteres são compostos por apenas um CC (geralmente assume-se vizinhança 8-conexa), comparar as características deles consiste em comparar atributos de nós próximos. Se assumirmos, por exemplo, que caracteres de uma mesma fonte têm alturas parecidas, podemos encontrar ruídos na imagem calculando a variância de nós da árvore que consistem da união de dois ou mais nós candidatos a letras:

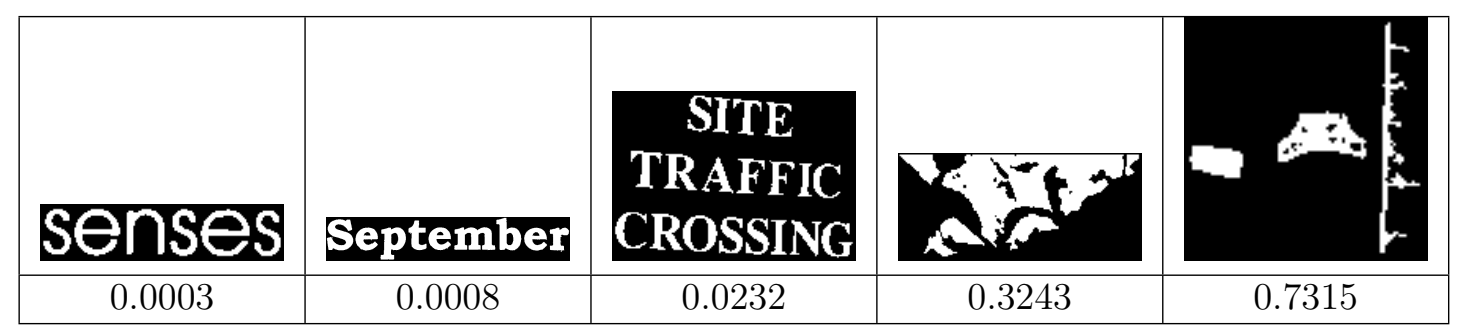

Tabela 5.1: Cálculo da variância da altura para alguns componentes conexos.

Como se pode ver nas imagens, em um dos componentes, várias linhas de texto se juntaram em uma só. No caso, como as alturas dos componentes ao longo das linhas ainda é parecido, a variância dos componentes não foi afetada. Para os resultados apresentados, um limiar seria suficiente para separar os ruídos das regiões de texto.

Atributos que combinam informações de dois nós originalmente disjuntos também podem ser úteis em localização de textos, além de filtragem de ruídos. Por exemplo, tendo as caixas envolventes (bounding boxes) de cada região candidata, é possível calcular o espaçamento, entre duas regiões, isto é, a distância horizontal as suas caixas envolventes. Assumindo que letras de uma mesma palavra têm espaçamento aproximadamente constante, podemos eliminar ruídos ou separar componentes que consistem apenas de palavras de componentes que contém toda uma frase calculando a variância 
dos espaçamentos das regiões candidatas. Neste caso, suponha que queremos extrair as palavras da imagem apresentada na Fig. 5.1.

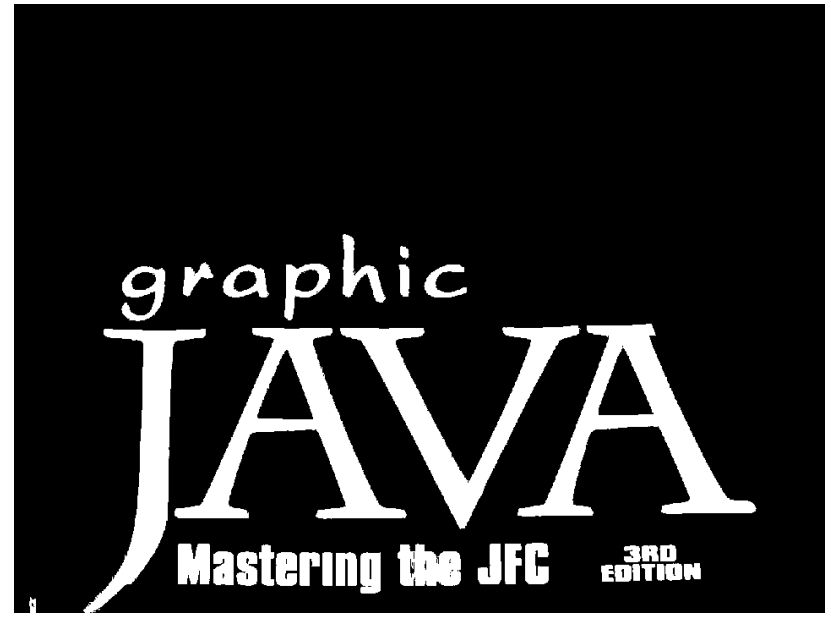

Figura 5.1: Imagem binária a fins de exemplo.

Na figura seguinte (Fig. 5.2), são mostradas as imagens das max-trees desta imagem para as vizinhanças 8x3, 16x3 e 32x3. Estas vizinhanças foram escolhidas de forma a termos, de forma aproximada, árvores cujas larguras dobram quando navegamos entre elas.

Os valores mostrados ao lado de cada nó indica a variância dos espaçamentos de cada um deles.

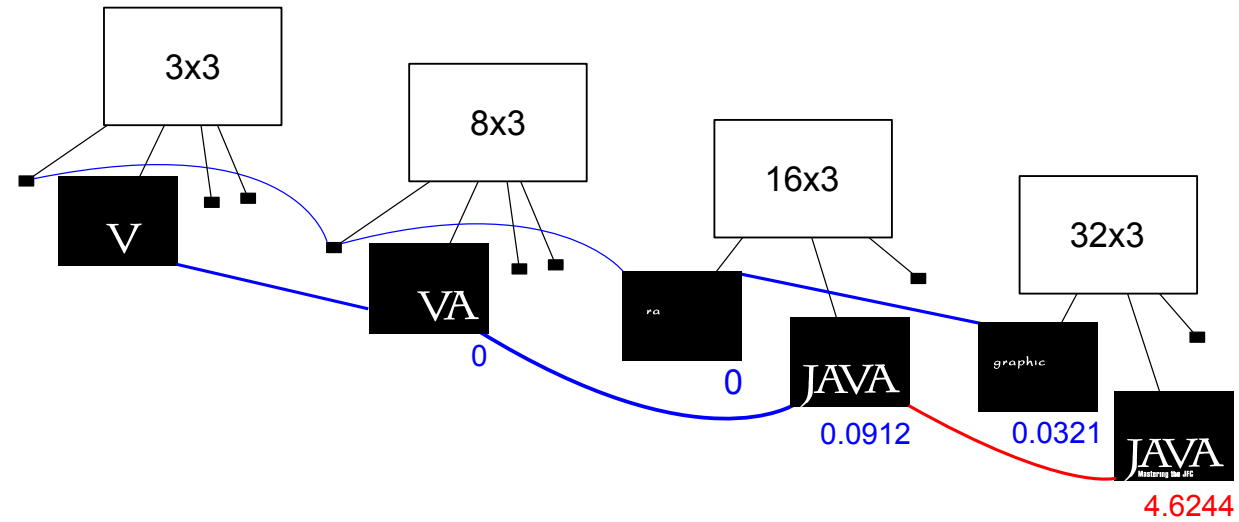

Figura 5.2: Alguns componentes conexos de diferentes árvores para a imagem anterior.

Os nós pequenos são usados apenas para indicar que existem mais nós na árvores que não estão sendo explicitamente mostrados. Para este exemplo em particular, não faremos uso deles.

Suponha que temos marcadas as letras da imagem. por exemplo, suponha que sabemos qual o componente da árvore 3x3 que contém a letra "v" da palavra "Java". Então, uma forma de encontrar a maior palavra que contém esta letra é navegar ao longo dos nós das árvores que contém a letra "v" até encontrarmos um nó com variância muito alta, que indica que ruídos ou um espaçamento muito diferente foi encontrado.

No exemplo, ao passarmos para a árvore com vizinhança 8x3, encontramos o nó que contém as letras "va". Depois, na árvore sob vizinhança 16x3, encontramos um CC que contém toda a palavra "Java". Assumindo que não sabemos de antemão que a palavra toda já foi encontrada, devemos continuar o processo navegando para a próxima árvore, comparando as variâncias até encontrarmos algum nó que ultrapassa algum limiar ou se não houver mais nós para navegarmos. No caso, por exemplo, se assumirmos que o limiar máximo aceito para espaçamentos dentro de uma mesma palavra é 0,1 , não avançaríamos para a árvore sob vizinhança 32x3 e teríamos a palavra "Java" encontrada corretamente.

Como este processo independe do valor de $i$ ou do tamanho da vizinhança, é possível encontrar 
palavrar com diferentes espaçamentos: por exemplo, a palavra "graphic" só se junta em um único componente na árvore sob vizinhança 32x3, mas apesar dos espaçamentos serem maiores, a variação entre eles é pequena. Considerando o mesmo limiar 0,1 anterior, o nó que contém a palavra também seria corretamente selecionado se tivermos alguma das letras da palavra definida como uma letra na primeira árvore.

\subsection{Consumo de tempo}

Esta seção apresenta alguns resultados obtidos através da teoria e dos algoritmos vistos ao longo do texto, com relação principalmente ao consumo de tempo do algoritmo. A fim de analisar o quão eficiente é o algoritmo neste quesito, foi também implementado o algoritmo visto de Berger et al. [BGL $\left.{ }^{+} 07\right]$. A razão pelo qual escolhemos a implementação deste algoritmo para comparação é que usamos a mesma estrutura de dados do trabalho deles para representar as relações de parentesco.

Os algoritmos foram implementados em Java e testados em um notebook com processador i5 $2.5 \mathrm{GHz}$ e $4 \mathrm{~GB}$ de RAM.

\subsubsection{Atualização das relações de parentesco}

De acordo com os autores, o algoritmo deles tem um comportamento quasi-linear, devido principalmente ao uso da estrutura que eles chamam de zpar. Tal estrutura age da mesma forma que o algoritmo de compressão de caminhos dos conjuntos disjuntos, porém com a utilidade de achar rapidamente o último ancestral de um nó durante a execução do algoritmo.

Para poder ser utilizada, esta estrutura requer que as relações de parentescos possam ser atualizadas de forma ordenada. Sabemos que o nosso algoritmo não satisfaz tal condição para os passos $i>1$. Portanto, o custo da atribuição de qual nó é pai do pixel atual é mais lenta comparada ao algoritmo deles.

Por outro lado, a suposição de complexidade quasi-linear só vale se supormos que a vizinhança passada tem tamanho constante. No nosso caso, como estamos interessados em construir árvores com vizinhanças cada vez maiores, espera-se que a complexidade do algoritmo de Berger et.al. aumente proporcionalmente de acordo com o tamanho da vizinhança passada.

A fim de comparar as duas abordagens, vamos inicialmente analisar o número de verificações feitas, isto é, quando pares de pixels precisam ser verificados para se atualizar as relações de parentesco da árvore. Esta análise será feita tomando duas imagens do banco de dados da conferência ICDAR[LPS ${ }^{+}$03], sob duas sequências de vizinhanças diferentes. A primeira imagem e a primeira sequência de vizinhanças escolhidas podem ser vistas na Fig. 5.3. A escolha das vizinhanças é feita para que a sequência gerada $\mathbb{A}$ cresça na horizontal, ou seja, a vizinhança $\mathcal{A}_{i}$ consiste em uma janela largura de $2 i+1$ e altura 3 com origem no centro.
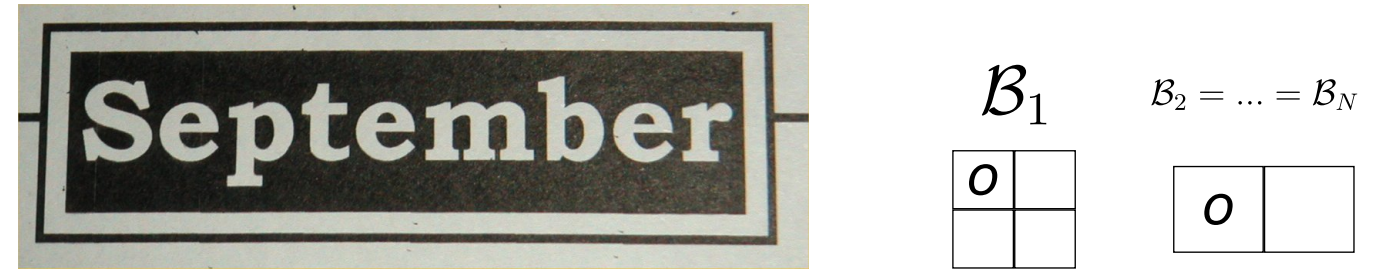

Figura 5.3: Imagem e vizinhança usadas para o primeiro teste de consumo de tempo.

Na Fig. 5.4, é exibido o gráfico mostrando o número de verificações que é feita por cada algoritmo, variando o valor de $i$. Nosso algoritmo é referido com a legenda "proposto", enquanto que o algoritmo de Berger et al. é denotado com a legenda "Berger".

Devido à forma com que o algoritmo de Berger et al. é construído, o número de verificações independe da topografia da imagem e depende apenas dos tamanhos da imagem e da vizinhança. Assim sendo, como o tamanho da vizinhança cresce linearmente (devido à escolha de $\mathcal{A}_{i}$ para $i \geq 2$ ), o número de verificações também cresce linearmente. 


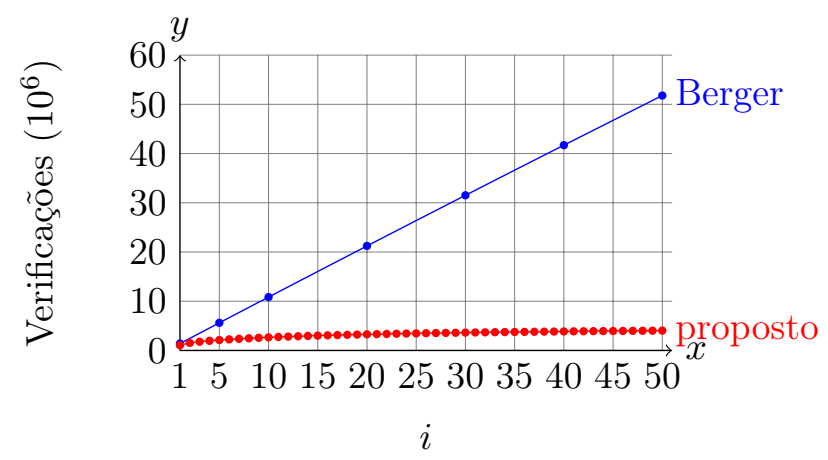

Figura 5.4: Número de verificações de cada algoritmo para a imagem da Fig. 5.3.

Como se pode ver no gráfico, o número de verificações feitas no nosso algoritmo é, de fato, bem menor, devido principalmente ao comportamento cíclico das vizinhanças, o que limita o número de vezes que cada pixel pode ser propagado. Além disso, como um pixel só pode ser propagado novamente quando um vizinho de nível maior o visita, o número de verificações diminui conforme $i$ aumenta, fazendo com o que a curva do número de verificações para o nosso algoritmo tenha um comportamento sublinear.

Como a etapa mais custosa de ambos os algoritmos é a verificação de alteração nas relações de parentesco, o tempo de execução é diretamente proporcional ao número de verificações, como se pode ser no gráfico a seguir.

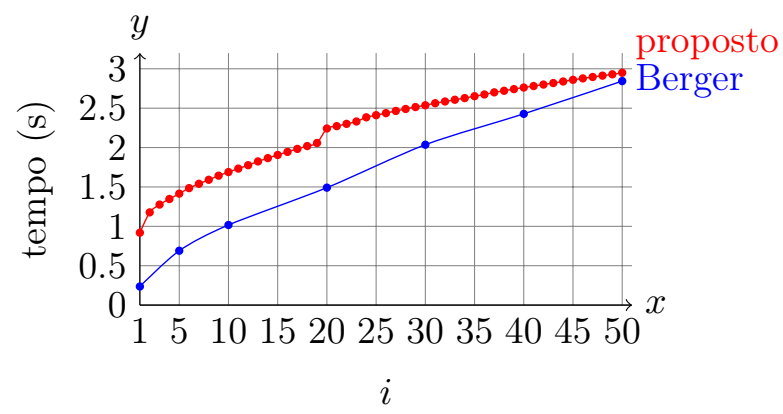

Figura 5.5: Consumo de tempo de cada algoritmo para a imagem da Fig. 5.3.

O gráfico anterior mostra que, apesar do número de comparações feitas pelo nosso algoritmo ser bem menor, para os valores de $i$ daquele intervalo, o algoritmo ainda consome mais tempo, em razão de não podermos comprimir os caminhos na busca dos nós ancestrais na atualização das relações de parentesco. Por outro lado, para valores de $i$ altos, o nosso algoritmo se torna mais rápido que o algoritmo de Berger et al.. Nesta imagem e sequência de vizinhanças $\mathbb{A}$ em particular, nosso algoritmo se torna mais eficiente por volta do passo $i=140$.

Por depender principalmente da topografia da imagem, o algoritmo aqui apresentado se torna particularmente mais eficiente nos casos em que o tamanho da vizinhança cresce de forma mais rápida que uma função linear. Assim sendo, consideraremos no segundo teste a imagem e sequência $\mathbb{A}$ exibidas na Fig. 5.6. Para este segundo teste, exibiremos mais uma curva em cada gráfico, a fim de comparar o desempenho do algoritmo proposto com e sem a suposição de que sabemos que a sequência de vizinhanças passada possui ciclos. Para tanto, simulamos o algoritmo com tamanho de ciclo 1 e $N$. No primeiro caso, é possível que alguns pixels não sejam re-adicionados à fila de prioridades e o número de verificações terá um comportamento sublinear com relação a $N$. No segundo caso, todos os pixels da imagem são adicionados à fila de prioridades durante todos os passo $i$, fazendo que o número de verificações tenha um comportamento linear em $N$.

Os resultados do algoritmo com a suposição cíclica são apresentadas nos gráficos através da curva de cor vermelha, com a legenda "com ciclo", enquanto que os resultados do algoritmo sem a suposição cíclica são apresentadas através da curva de cor cinza, com a legenda "sem ciclo". Uma 
análise mais detalhada com relação à complexidade esperada do algoritmo é apresentada na Seção 5.3 .

Conforme podemos ver no gráfico da Fig. 5.7, o nosso algoritmo já se torna mais eficiente que o algoritmo de Berger et.al. mesmo para valores de $i$ não tão grandes. Além disso, também se é possível observar como a suposição cíclica impacta em uma redução no número de verificações necessárias para se atualizar as relações de parentesco.

No gráfico da Fig. 5.8, que trata de valores $i$ maiores, é possível ver as curvas de desempenho de cada algoritmo com relação ao número de verificações e o tempo de execução.
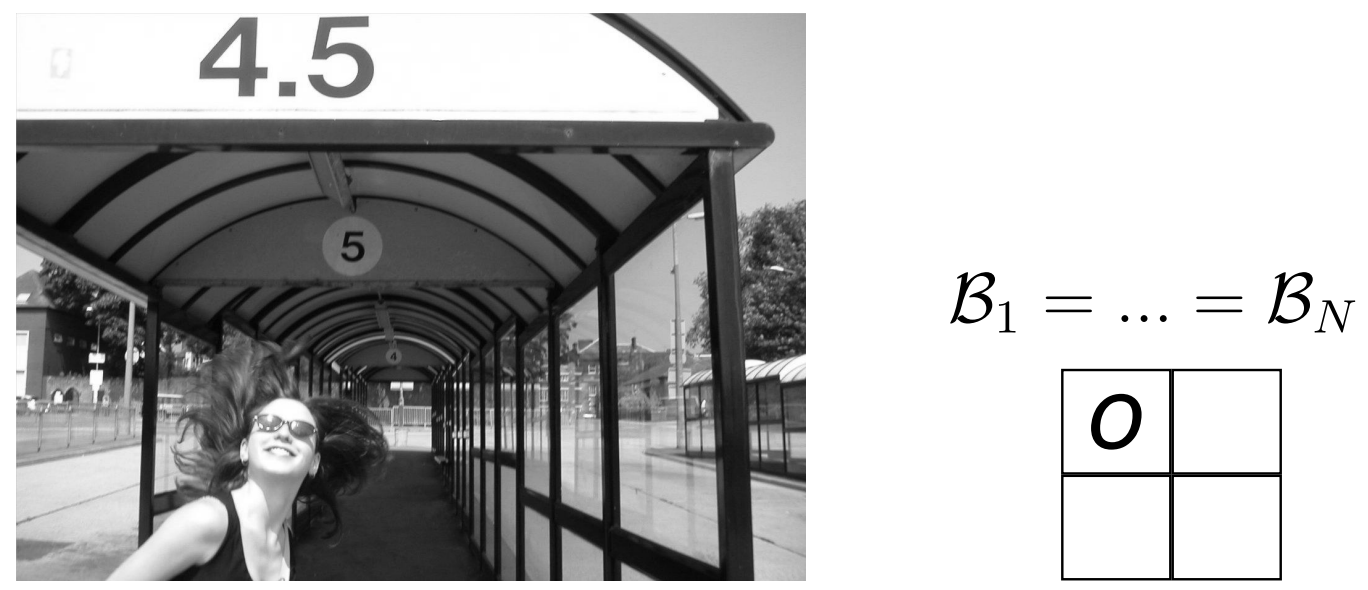

Figura 5.6: Imagem e vizinhança usadas para o segundo teste de consumo de tempo.
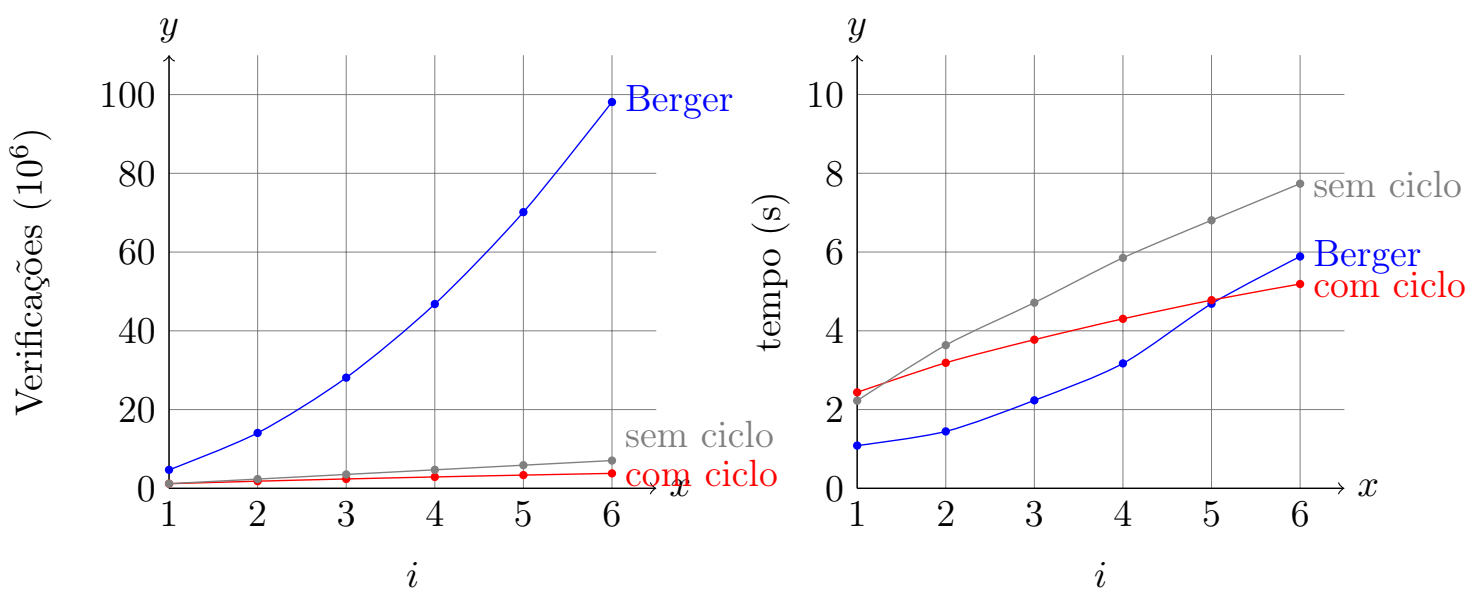

Figura 5.7: Número de verificações e consumo de tempo para a Fig. 5.3.

É importante lembrar que, além de atualizar as relações de parentesco, nosso algoritmo armazena todas as relações modificadas em uma lista, o que não ocorre no algoritmo de Berger et al.. Isso permite atualizar a árvore para qualquer $i$ menor que o máximo passado no início do programa. Por exemplo, se rodarmos o algoritmo para o índice $N=100$, poderemos reconstruir qualquer árvore com índice $i$ entre 0 e 100, bastando percorrer a lista e atualizar as relações de parentesco para os pares de pixels que se uniram até o passo $i$.

\subsubsection{Algoritmos posteriores}

Uma vez que tenhamos a estrutura com todas as atualizações de parentesco construída, podemos construir a max-tree de qualquer vizinhança com índice menor ou igual ao passado na etapa anterior.

Usando as mesmas imagens e vizinhanças das Figs 5.3 e 5.6, foram aplicados os seguintes algoritmos às imagens, em sequência: 

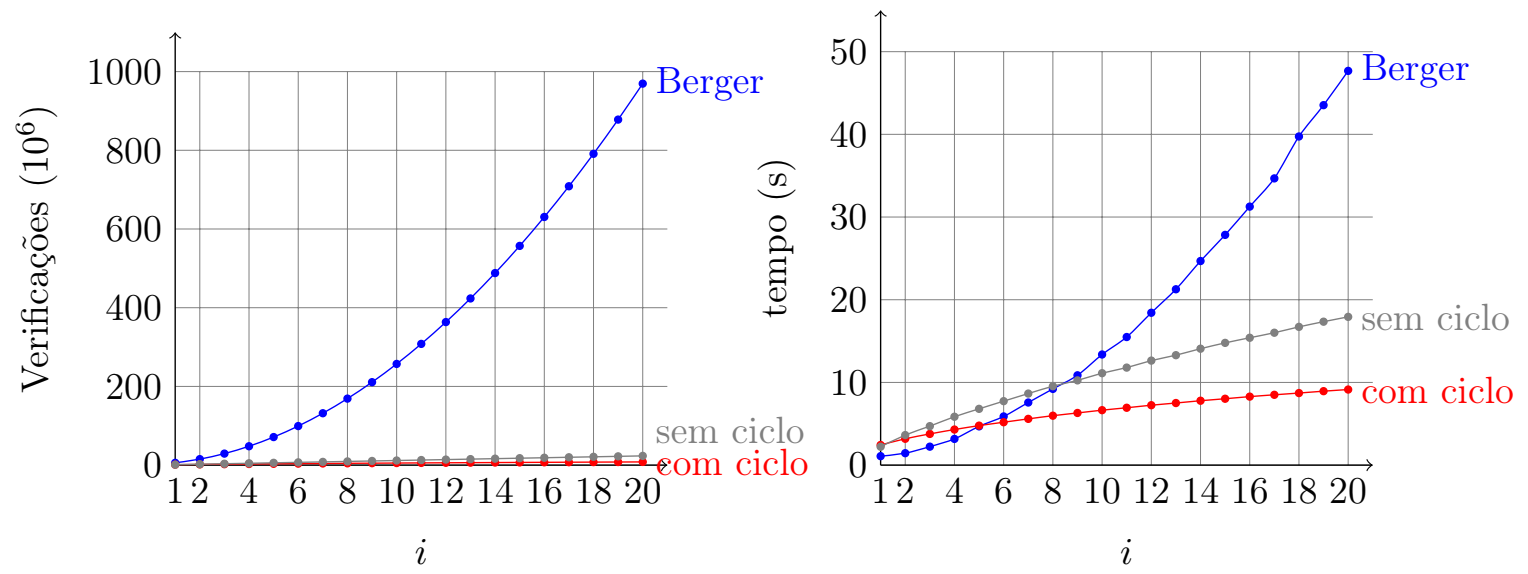

Figura 5.8: Número de verificações e consumo de tempo para a Fig. 5.6.

1. Atualização: consiste em alocar um vetor de parentesco vazio e atualizá-lo para o valor $i$ passado como parâmetro;

2. Max-tree: consiste em alocar os nós e atualizar as relações de parentesco e calcular os atributos de todos os nós da árvore usando o vetor obtido no algoritmo anterior;

3. Inclusão: consiste do algoritmo atualizaCCsOriginais, responsável pela inclusão dos nós de diferentes árvores. O nó menor passado nos testes é o nó raiz da árvore obtida pela vizinhança $\mathcal{A}_{1}$ (por isso só é necessário rodar este algoritmo para índice maiores que 1);

4. Atributos: é o algoritmo que calcula a variância dos atributos dos nós da árvore menor que estão contidos nos nós maiores. Neste teste, foi atualizado o atributo altura dos nós;

Os resultados dos tempos de execução de cada algoritmo para cada imagens por ser vistas nas tabelas a seguir:

\begin{tabular}{|l|l|l|l|l|l|}
\hline$i$ & Atualização & Max-tree & Inclusão & Atributos & Total \\
\hline 1 & $0.045 \mathrm{~s}$ & $0.503 \mathrm{~s}$ & & & $0.548 \mathrm{~s}$ \\
\hline 2 & $0.091 \mathrm{~s}$ & $0.288 \mathrm{~s}$ & $0.039 \mathrm{~s}$ & $0.038 \mathrm{~s}$ & $0.457 \mathrm{~s}$ \\
\hline 4 & $0.053 \mathrm{~s}$ & $0.264 \mathrm{~s}$ & $0.074 \mathrm{~s}$ & $0.009 \mathrm{~s}$ & $0.400 \mathrm{~s}$ \\
\hline 8 & $0.053 \mathrm{~s}$ & $0.371 \mathrm{~s}$ & $0.074 \mathrm{~s}$ & $0.005 \mathrm{~s}$ & $0.503 \mathrm{~s}$ \\
\hline 16 & $0.053 \mathrm{~s}$ & $0.236 \mathrm{~s}$ & $0.011 \mathrm{~s}$ & $0.004 \mathrm{~s}$ & $0.304 \mathrm{~s}$ \\
\hline 32 & $0.056 \mathrm{~s}$ & $0.382 \mathrm{~s}$ & $0.011 \mathrm{~s}$ & $0.008 \mathrm{~s}$ & $0.457 \mathrm{~s}$ \\
\hline 64 & $0.046 \mathrm{~s}$ & $0.233 \mathrm{~s}$ & $0.010 \mathrm{~s}$ & $0.004 \mathrm{~s}$ & $0.293 \mathrm{~s}$ \\
\hline 128 & $0.061 \mathrm{~s}$ & $0.272 \mathrm{~s}$ & $0.010 \mathrm{~s}$ & $0.003 \mathrm{~s}$ & $0.346 \mathrm{~s}$ \\
\hline
\end{tabular}

Tabela 5.2: Consumo de tempo para cada algoritmo com relação à Fig. 5.3.

Como se pode observar no gráfico, todas as etapas são bem rápidas. Conforme o índice $i$ aumenta, o número de nós diminui, fazendo com que todos os passos após a atualização das relações de parentesco fiquem mais rápidas, principalmente no que diz respeito à construção da max-tree, que envolve alocação de memória.

Como o número de verificações cresce de forma bem lenta para valores altos de $i$, o passo de atualização das relações de parentesco não muda muito em comparação a índices pequenos. 


\begin{tabular}{|l|l|l|l|l|l|}
\hline$i$ & Atualização & Max-tree & Inclusão & Atributos & Total \\
\hline 1 & $0.014 \mathrm{~s}$ & $0.132 \mathrm{~s}$ & & & $0.146 \mathrm{~s}$ \\
\hline 2 & $0.011 \mathrm{~s}$ & $0.110 \mathrm{~s}$ & $0.011 \mathrm{~s}$ & $0.006 \mathrm{~s}$ & $0.139 \mathrm{~s}$ \\
\hline 4 & $0.013 \mathrm{~s}$ & $0.097 \mathrm{~s}$ & $0.011 \mathrm{~s}$ & $0.008 \mathrm{~s}$ & $0.129 \mathrm{~s}$ \\
\hline 8 & $0.014 \mathrm{~s}$ & $0.093 \mathrm{~s}$ & $0.010 \mathrm{~s}$ & $0.005 \mathrm{~s}$ & $0.122 \mathrm{~s}$ \\
\hline 16 & $0.013 \mathrm{~s}$ & $0.077 \mathrm{~s}$ & $0.008 \mathrm{~s}$ & $0.004 \mathrm{~s}$ & $0.102 \mathrm{~s}$ \\
\hline 32 & $0.013 \mathrm{~s}$ & $0.068 \mathrm{~s}$ & $0.010 \mathrm{~s}$ & $0.003 \mathrm{~s}$ & $0.094 \mathrm{~s}$ \\
\hline 64 & $0.012 \mathrm{~s}$ & $0.080 \mathrm{~s}$ & $0.010 \mathrm{~s}$ & $0.003 \mathrm{~s}$ & $0.105 \mathrm{~s}$ \\
\hline 128 & $0.014 \mathrm{~s}$ & $0.066 \mathrm{~s}$ & $0.009 \mathrm{~s}$ & $0.002 \mathrm{~s}$ & $0.091 \mathrm{~s}$ \\
\hline
\end{tabular}

Tabela 5.3: Consumo de tempo para cada algoritmo com relação à Fig. 5.6.

\subsection{Complexidade esperada do algoritmo de construção das max- trees}

O custo de algoritmos usados para a construção de max-trees está diretamente relacionada ao número de pares de pixels que são necessários avaliar para se atualizar a relação de parentesco. No nosso caso, é difícil calcular a complexidade exata do algoritmo porque a função de união depende diretamente da topologia da imagem de entrada. Além disso, a condição cíclica dos elementos estruturantes pode reduzir a complexidade do algoritmo ao evitarmos re-adicionar elementos redundantes para serem processados nos passos seguintes.

Numa primeira análise, considere o caso acíclico. Neste caso, os parâmetros que influenciam na complexidade para cada passo $i$ são os seguintes: o número de elementos na fila $\left(|\mathcal{D}|=n^{2}\right.$, onde $n^{2}$ é o tamanho da imagem de entrada), o número de vizinhos por pixel (limitado por $\left|\mathcal{B}_{i}\right|$ ), e o custo do algoritmo de união, cujo custo é o tamanho do caminho ligando os nós que estamos tentando juntar (ou seja, é limitado superiormente por $2 K$, onde $K$ é o número de níveis de cinza diferentes). Supondo que o algoritmo de ordenação tenha complexidade $O\left(n^{2} \log (n)\right)$, uma análise de pior caso nos dá uma complexidade ou de $O\left(N n^{2} B K\right)$, onde $B=\max _{i \in I}\left|\mathcal{B}_{i}\right|$, ou de $O\left(N n^{2} \log (n)\right)$, dependendo de como $B K$ se compara a $\log (n)$.

O caso cíclico é mais complexo e, neste caso, vamos fazer uma análise de complexidade esperada apenas para o caso específico em que o tamanho do ciclo é 1 e a sequência $\mathbb{B}$ considerada é a sequência usada nos testes da Fig. 5.6. Neste caso, o que muda na complexidade é que o número de elementos na fila pode ser menor que $n^{2}$. Como este parâmetro depende da topologia da imagem, vamos considerar o caso em que $f$ é uma imagem aleatória onde cada pixel recebe um valor entre 0 e $K-1$ aleatoriamente com probabilidade uniforme.

No caso particular da sequência $\mathbb{B}$ escolhida, precisamos calcular a probabilidade de um pixel qualquer $p$ ser re-adicionado à fila no passo seguinte. Para o passo $i=1$, existem 3 pixels que podem alterar o rótulo de $p$ : os pixels do conjunto $p \oplus\left(\mathcal{B}_{1}^{\Sigma}\right)^{t} \backslash\{p\}$. Pela construção do algoritmo, um rótulo só é alterado se algum dos vizinhos tem nível de cinza maior que $f(p)$. No caso em que $K \rightarrow \infty$, a probabilidade de que algum vizinho tenha nível de cinza maior tende a $\frac{3}{4}$. Porém, como $K$ é finito e um eventual empate de níveis de cinza não fará com que $p$ seja re-adicionado à fila, podemos afirmar que esta probabilidade é limitada superiormente por $\frac{3}{4}$.

Para o passo $i=2$, os elementos que potencialmente poderiam alterar o rótulo de $p$ são os vizinhos em $p \oplus\left(\mathcal{B}_{2}^{\Sigma}\right)^{t}$. Porém, os vizinhos em $p \oplus\left(\mathcal{B}_{1}^{\Sigma}\right)^{t}$ são um subconjunto de $p \oplus\left(\mathcal{B}_{2}^{\Sigma}\right)^{t}$ e já foram considerados no passo anterior. Assim, se o elemento de maior valor em $p \oplus\left(\mathcal{B}_{2}^{\Sigma}\right)^{t}$ estiver também em $p \oplus\left(\mathcal{B}_{1}^{\Sigma}\right)^{t}$, o rótulo de $p$ não será alterado pois nenhum elemento de $\left(p \oplus\left(\mathcal{B}_{2}^{\Sigma}\right)^{t}\right) \backslash\left(p \oplus \mathcal{B}_{1}^{t}\right)$ possui nível de cinza superior ao elemento que já rotula $p$ desde o passo $i=1$. Portanto, para $i=2$, podemos afirmar que a probabilidade de que $p$ seja re-adicionado à fila é limitado pela razão entre o número de vizinhos de $p$ sob $\left(\mathcal{B}_{1}^{\Sigma}\right)^{t}$ e o número de vizinhos de $p \operatorname{sob}\left(\mathcal{B}_{2}^{t}\right)^{\Sigma}$, ou seja, é menor ou igual a $\frac{5}{9}$.

Se continuarmos com o mesmo raciocínio, é fácil verificar que, para qualquer $i$, esta razão é igual 
a $\frac{(i+1)^{2}-i^{2}}{(i+1)^{2}}=\frac{2 i+1}{(i+1)^{2}}<\frac{2(i+1)}{(i+1)^{2}}=2 \frac{1}{i+1}$. Assim, o numero esperado de vezes que um mesmo elemento é adicionado à fila consiste em somar esta fração para os valores $i$ de 1 até $N$ :

$$
\begin{array}{r}
\sum_{i=1}^{N} \frac{2}{i+1} \\
=2 \sum_{i=1}^{N} \frac{1}{i+1} \\
=2\left(\sum_{i=1}^{N+1} \frac{1}{i}-1\right) \\
\leq 2 \ln (N+1) \\
=2\left(\ln N+\ln \left(1+\frac{1}{N}\right)\right) \\
\leq 2+2(\ln (N)+1)
\end{array}
$$

A primeira desigualdade acima surge por causa de uma propriedade da série harmônica $\left(\sum_{i=1}^{N} \frac{1}{i} \leq\right.$ $1+\ln N)$ e o último passo vale porque $N \geq 1$ implica $1+\frac{1}{N} \leq e \Rightarrow \ln \left(1+\frac{1}{N}\right) \leq 1$.

Assim, o número esperado de vezes que um elemento é adicionado à fila é limitado superiormente por uma função logarítmica em $N$, o que reduz a primeira complexidade (sem considerar o custo de ordenação) de $O\left(N n^{2} B K\right)$ para $O\left(n^{2} \ln (N) B K\right)$ (na realidade $|B|=4$ é constante neste caso).

Resta calcular o custo esperado da etapa de ordenação para o algoritmo durante todos os passos de 1 a $N$. Então, a complexidade esperada para a ordenação em todos os passos $i$ pode ser estimada como:

$$
\begin{array}{r}
\sum_{i=1}^{N} \frac{2 n^{2}}{i+1} \log \left(\frac{2 n^{2}}{i+1}\right) \\
=2 n^{2} \sum_{i=1}^{N} \frac{1}{i+1}\left(\log \left(2 n^{2}\right)-\log (i+1)\right) \\
=2 n^{2} \log \left(2 n^{2}\right) \sum_{i=1}^{N} \frac{1}{i+1}-2 n^{2} \sum \frac{\log (i+1)}{i+1} \\
\leq 2 n^{2} \times 2(\log (n)+\log (2)) \times 2(\ln (N)+1) \\
\leq 8 n^{2} \times \log (n) \times \ln (N)+4
\end{array}
$$

Portanto, o custo de ordenação total tem complexidade esperada $O\left(n^{2} \log (n) \log (N)\right)$ e a complexidade esperada para este caso específico reduz o parâmetro $N$ para uma função logarítmica em $N$ tanto na etapa de construção quanto na etapa de ordenação.

Para este caso específico, a complexidade obtida é de $O\left(n^{2} \log (N)(\log (n)+K)\right)$ e esta de acordo com as curvas obtidas no teste da Fig. 5.6: conforme aumentamos $N$ linearmente, o consumo de tempo cresce de forma sublinear e logarítmica em $N$. Isso mostra que a consideração cíclica pode reduzir consideravelmente o tempo de execução do algoritmo, reduzindo os termos multiplicativos que eram lineares em $N$ para um fator que é logarítmico em $N$, o que permite a construção das árvores mesmo com vizinhanças grandes, que não seriam praticáveis com os algoritmos tradicionais. 


\section{Capítulo 6}

\section{Considerações Finais}

Concluída a apresentação da teoria e dos algoritmos desenvolvidos nesta dissertação, apresentamos neste último capítulo os próximos passos com relação aos temas que podem ser estudados a fim de estender este trabalho e as palavras finais que concluem as principais contribuições desta dissertação.

\subsection{Próximos passos}

Dentre os tópicos que não foi possível explorar nesta dissertação e que permitem uma continuidade do estudo, alguns pontos que podem ser destacados são:

- O desenvolvimento de outras aplicações que podem ser beneficiadas pelo uso da sequência de árvores;

- Um estudo mais aprofundado das vizinhanças que podem ser geradas a partir das condições dadas e analisar se existe uma classe ainda mais geral de vizinhanças que pode ser beneficiada pela teoria apresentada neste trabalho.

- Uma análise se é necessária alterações na teoria para o uso dessas árvores em imagens tridimensionais.

Um ponto importante que seria importante considerar em um trabalho futuro consiste em comparar o algoritmo proposto com o algoritmo de Ouzounis e Wilkinson [OW07], que foi desenvolvido especificamente para conectividades de segunda geração. Este algoritmo tem uma complexidade esperada melhor que o algoritmo que foi comparado( [BGL $\left.\left.{ }^{+} 07\right]\right)$ nos testes de tempo de execução, mas um estudo mais aprofundado seria necessário para saber como gerar as imagens máscaras que são requeridas para gerar a mesma sequência de vizinhanças que estamos usando em nosso algoritmo.

\subsection{Conclusão}

Neste trabalho foi explorada a ideia do uso de uma sequência de max-trees para representar CCs de uma imagem em diferentes vizinhanças, permitindo estabelecer relações entre nós de diferentes árvores. Uma teoria foi desenvolvida para o uso de uma sequência de vizinhanças que podem ser obtidas a partir de dilatações e, a partir desta teoria, um novo algoritmo foi introduzido, permitindo a construção das árvores associadas a estas vizinhanças de forma eficiente. Para mostrar a eficiência do nosso algoritmo, testamos-o em imagens reais e mostramos que o nosso algoritmo não apenas tem um bom desempenho quando consideramos vizinhanças pequenas, mas que também ele permite a construção de árvores sob vizinhanças grandes, o que não seria factível considerando os algoritmos usuais de construção de max-trees. 
Apêndice 



\section{Apêndice A}

\section{Lista de Publicações}

- Alexandre Morimitsu, Wonder Alexandre Luz Alves e Ronaldo Fumio Hashimoto. Incremental and Efficient Computation of Families of Component Trees. Em Benediktsson, J.A., Chanussot, J., Najman, L., Talbot, H. (eds.) ISMM 2015. volume 9082, páginas 681-692. Springer, Heidelberg, 2015.

- Wonder Alexandre Luz Alves, Alexandre Morimitsu e Ronaldo Fumio Hashimoto. Scale-Space Representation Based on Levelings Through Hierarchies of Level Sets. Em Benediktsson, J.A., Chanussot, J., Najman, L., Talbot, H. (eds.) ISMM 2015. volume 9082, páginas 265-276. Springer, Heidelberg, 2015.

- Wonder Alexandre Luz Alves, Alexandre Morimitsu, Joel Sánchez Castro e Ronaldo Fumio Hashimoto. Extraction of numerical residues in families of levelings. Em Graphics, Patterns and Images (SIBGRAPI), 2013 26th SIBGRAPI-Conference, páginas 349-356. IEEE, 2013. 


\section{Referências Bibliográficas}

[AH10] Wonder Alexandre Luz Alves e Ronaldo Fumio Hashimoto. Text regions extracted from scene images by ultimate attribute opening and decision tree classification. Em Graphics, Patterns and Images (SIBGRAPI), 2010 23rd SIBGRAPI Conference on, páginas 360-367. IEEE, 2010. 4

[BGL ${ }^{+}$07] Ch Berger, T. Géraud, R. Levillain, N. Widynski, A. Baillard e E. Bertin. Effective component tree computation with application to pattern recognition in astronomical imaging. Em Image Processing, 200\%. ICIP 200\%. IEEE International Conference on, volume 4, páginas IV - 41-IV - 44, 2007. 4, 27, 29, 45, 53, 59

[BNG03] Ulisses Braga-Neto e John Goutsias. A theoretical tour of connectivity in image processing and analysis. Journal of Mathematical Imaging and Vision, 19(1):5-31, 2003. 4

[CG13] Edwin Carlinet e Thierry Géraud. A comparison of many max-tree computation algorithms. Em Mathematical Morphology and Its Applications to Signal and Image Processing, páginas 73-85. Springer, 2013. 4

[DLfOES03] Edward R Dougherty, Roberto A Lotufo e The International Society for Optical Engineering SPIE. Hands-on morphological image processing, volume 71. SPIE press Bellingham, 2003. 7

[FKW11] Paulo Feofiloff, Yoshiharu Kohayakawa e Yoshiko Wakabayashi. Uma introdução sucinta à teoria dos grafos. Disponıvel em http://www. ime. usp. br/ ${ }^{\sim} \mathrm{pf} /$ teoriadosgrafos, 2011. 9

[HM11] Jorge Hernández e Beatriz Marcotegui. Shape ultimate attribute opening. Image and Vision Computing, 29(8):533-545, 2011. 4

[Jon99] Ronald Jones. Connected filtering and segmentation using component trees. Computer Vision and Image Understanding, 75(3):215-228, 1999. 4

[LMGN05] Miriam León, Sergio Mallo, Antoni Gasull e UPC-Campus Nord. A tree structuredbased caption text detection approach. Em Proceedings of the Fifth IASTED International Conference on Visualization, Imaging, and Image Processing, página 220, 2005. 4

[LPS ${ }^{+}$03] Simon M Lucas, Alex Panaretos, Luis Sosa, Anthony Tang, Shirley Wong e Robert Young. Icdar 2003 robust reading competitions. Em ICDAR, volume 2003, página 682, 2003. 53

[MG00] Pascal Monasse e Frederic Guichard. Fast computation of a contrast-invariant image representation. Image Processing, IEEE Transactions on, 9(5):860-872, 2000. 11

[MM13] Stefano Messelodi e Carla Maria Modena. Scene text recognition and tracking to identify athletes in sport videos. Multimedia Tools and Applications, páginas 1-25, 2013. 4 
[NC06] L. Najman e M. Couprie. Building the component tree in quasi-linear time. Image Processing, IEEE Transactions on, 15(11):3531-3539, Nov 2006. 4, 27

[NM12] Lukas Neumann e Jiri Matas. Real-time scene text localization and recognition. Em Computer Vision and Pattern Recognition (CVPR), 2012 IEEE Conference on, páginas 3538-3545. IEEE, 2012. 4

[OW07] G. K. Ouzounis e M. H. F. Wilkinson. Mask-based second generation connectivity and attribute filters. IEEE Trans. Pattern Anal. Mach. Intell., 29:990-1004, 2007. 4, 59

[PN11] Nicolas Passat e Benoît Naegel. Component-hypertrees for image segmentation. Em Mathematical Morphology and Its Applications to Image and Signal Processing, páginas 284-295. Springer, 2011. 1, 4

[RM07] Thomas Retornaz e Beatriz Marcotegui. Scene text localization based on the ultimate opening. Em International Symposium on Mathematical Morphology, volume 1, páginas 177-188, 2007. 2, 4

[Ser98] Jean Serra. Connectivity on complete lattices. Journal of Mathematical Imaging and Vision, 9(3):231-251, 1998. 4

[SG00] Philippe Salembier e Luis Garrido. Connected operators based on region-tree pruning. Em Mathematical Morphology and its Applications to Image and Signal Processing, páginas 169-178. Springer, 2000. 4

[SH12] Lei Sun e Qiang Huo. A component-tree based method for user-intention guided text extraction. Em Pattern Recognition (ICPR), 2012 21st International Conference on, páginas 633-636. IEEE, 2012. 4

[SOG98] Philippe Salembier, Albert Oliveras e Luis Garrido. Antiextensive connected operators for image and sequence processing. Image Processing, IEEE Transactions on, 7(4):555570, 1998. 1, 4, 11

[SRML14] Roberto Souza, Leticia Rittner, Rubens Machado e Roberto Lotufo. Maximal max-tree simplification. Em Pattern Recognition (ICPR), 2014 22nd International Conference on, páginas 3132-3137. IEEE, 2014. 4

[SW09] Philippe Salembier e Michael HF Wilkinson. Connected operators. Signal Processing Magazine, IEEE, 26(6):136-157, 2009. 4

[Tar75] Robert Endre Tarjan. Efficiency of a good but not linear set union algorithm. Journal of the ACM (JACM), 22(2):215-225, 1975. 27

[WGH $\left.{ }^{+} 08\right]$ M. H. F. Wilkinson, H. Gao, W. H. Hesselink, J. E. Jonker e A. Meijster. Concurrent computation of attribute filters using shared memory parallel machines. IEEE Trans. Pattern Anal. Mach. Intell., 30(10):1800-1813, 2008. 4, 27, 33

[XGN12] Yongchao Xu, Thierry Géraud e Laurent Najman. Morphological filtering in shape spaces: Applications using tree-based image representations. Em Pattern Recognition (ICPR), 2012 21st International Conference on, páginas 485-488. IEEE, 2012. 4 


\section{Índice Remissivo}

Árvore, 9

de componentes, 10

enraizada no nó raiz, 9

Max-tree, 13

Min-tree, 13

Pixel, 9

de fundo, 10

de objeto, 10

Aresta, 9

Atributo, 13

Área, 14

Altura, 14

Centroide, 14

Espaçamento, 47

Máximo $x, 13$

Máximo $y, 13$

Mínimo $x, 13$

Mínimo $y, 13$

Variância, 42

Caminho, 9

$\mathrm{CC}$

comprimento de, 9

Representante, 26

Ciclo, 9

Componente conexo

de grafos, 9

em imagens, 10

Conjunto de nível, 10

inferior, 10

superior, 10

Conjuntos disjuntos, 26

Elemento canônico, 26

Representante, 26

Dilatação, 7

Elemento estruturante, 7 origem de um, 7

Floresta, 9

Função de Rotulação

binária, 17 em níveis de cinza, 21

Grafo, 9

de níveis maximais, 12

de níveis superiores, 11

conexo, 9

Incidência, 9

peso, 9

Ponta, 9

Imagem, 9

binária, 9

em níveis de cinza, 9

Intensidade, 9

Nó, 9

ancestral, 9

descendente, 9

filho, 9

folha, 9

pai, 9

raiz, 9

Propagação de rótulo, 17

Rotulação

Função de, 21

Grafo de, 20

Sequência

gerada $\mathbb{A}, 15$

geradora $\mathbb{B}, 15$

Subárvore enraizada em um nó, 9

Subgrafo, 9

induzido, 9

Transposta, 7

Vértice, 9

Vizinhança, 10

Vizinho, 9 